\title{
ON THE SINGULARITIES OF THE SZEGŐ PROJECTIONS ON LOWER ENERGY FORMS
}

\author{
CHIN-YU HSIAO AND GEORGE MARINESCU
}

Dedicated to our teachers Professors Louis Boutet de Monvel and Johannes Sjöstrand

\begin{abstract}
Let $X$ be an abstract not necessarily compact orientable CR manifold of dimension $2 n-1, n \geqslant 2$. Let $\square_{b}^{(q)}$ be the Gaffney extension of Kohn Laplacian for $(0, q)$-forms. We show that the spectral function of $\square_{b}^{(q)}$ admits a full asymptotic expansion on the non-degenerate part of the Levi form. As a corollary, we deduce that if $X$ is compact and the Levi form is nondegenerate of constant signature on $X$, then the spectrum of $\square_{b}^{(q)}$ in $] 0, \infty[$ consists of point eigenvalues of finite multiplicity. Moreover, we show that a certain microlocal conjugation of the associated Szegó kernel admits an asymptotic expansion under a local closed range condition. As applications, we establish the Szegő kernel asymptotic expansions on some weakly pseudoconvex CR manifolds and on CR manifolds with transversal CR $S^{1}$ actions. By using these asymptotics, we establish some local embedding theorems on CR manifolds and we give an analytic proof of a theorem of Lempert asserting that a compact strictly pseudoconvex CR manifold of dimension three with a transversal CR $S^{1}$ action can be CR embedded into $\mathbb{C}^{N}$, for some $N \in \mathbb{N}$.
\end{abstract}

\section{CONTENTS}

1. Introduction and statement of the main results

2. Preliminaries

2.1. Standard notations

2.2. Set up and Terminology

3. Microlocal Hodge decomposition Theorems for $\square_{b}^{(q)}$

4. Microlocal spectral theory for $\square_{b}^{(q)}$

5. Szegő kernel asymptotic expansions

6. Szegő projections on CR manifolds with transversal CR $S^{1}$ actions

7. Szegő kernel asymptotic expansion on weakly pseudoconvex CR manifolds

7.1. Compact pseudoconvex domains

7.2. Non-compact pseudoconvex domains

8. Proof of Theorem5.4

Acknowledgements

References

2010 Mathematics Subject Classification. 32V20 (primary), and 32V30, 32W10, 35H20 (secondary).

The first author was partially supported by Taiwan Ministry of Science of Technology project 103-2115-M001-001, the DFG project MA 2469/2-2 and funded through the Institutional Strategy of the University of Cologne within the German Excellence Initiative.

The second author partially supported by the DFG projects SFB/TR 12, MA 2469/2-1 and Université Paris 7. 


\section{INTRODUCTION AND STATEMENT OF THE MAIN RESULTS}

Let $\left(X, T^{1,0} X\right)$ be a CR manifold of hypersurface type and dimension $2 n-1, n \geq 2$. Let $\square_{b}^{(q)}$ be the Gaffney extension of the Kohn Lalpacian acting on $(0, q)$ forms. The orthogonal projection $\Pi^{(q)}: L_{(0, q)}^{2}(X) \rightarrow \operatorname{Ker} \square_{b}^{(q)}$ onto $\operatorname{Ker} \square_{b}^{(q)}$ is called the Szegö projection, while its distribution kernel $\Pi^{(q)}(x, y)$ is called the Szegő kernel. The study of the Szegő projection and kernel is a classical subject in several complex variables and CR geometry.

When the Levi form satisfies condition $Y(q)$ on $X$ (see Definition 2.2), then Kohn's subelliptic estimates with loss of one dervative for the solutions of $\square_{b}^{(q)} u=f$ hold, cf. [23, 34, 50], and hence $\Pi^{(q)}$ is a smoothing operator. When condition $Y(q)$ fails, one is interested in the singularities of the Szegö kernel $\Pi^{(q)}(x, y)$.

A very important case is when $X$ is a compact strictly pseudoconvex CR manifold (in this case $Y(0)$ fails). Assume first that $X$ is the boundary of a strictly pseudoconvex domain. Boutet de Monvel-Sjöstrand [17] showed that $\Pi^{(0)}(x, y)$ is a Fourier integral operator with complex phase. In particular, $\Pi^{(0)}(x, y)$ is smooth outside the diagonal of $X \times X$ and there is a precise description of the singularity on the diagonal $x=y$, where $\Pi^{(0)}(x, x)$ has a certain asymptotic expansion.

The Boutet de Monvel-Sjöstrand description of the Szegő kernel had a profound impact in many research areas, especially through [18]: several complex variables, symplectic and contact geometry, geometric quantization, Kähler geometry, semiclassical analysis, quantum chaos, cf. [11, 12, 13, [15, 16, 21, 22, 26, 29, 38, 40, 49, 54, [55, 64, 66], to quote just a few. These ideas also partly motivated the introduction of alternative approaches, see [55, 56, 58, 57].

From the works of Boutet de Monvel [14], Boutet de Monvel-Sjöstrand [17], HarveyLawson [39], Burns [19] and Kohn [51, 52] follows that the conditions below are equivalent for a compact strictly pseudoconvex CR manifold $X, \operatorname{dim}_{\mathbb{R}} X \geqslant 3$ :

(a) $X$ is embeddable in the Euclidean space $\mathbb{C}^{N}$, for $N$ sufficiently large;

(b) $X$ bounds a strictly pseudoconvex complex manifold;

(c) The Kohn Laplacian $\square_{b}^{(0)}$ on functions of $X$ has closed range in $L^{2}$.

Therefore the description of the Szegő kernel given by [17] holds for CR manifolds satisfying the equivalent conditions (a)-(c). Moreover, if $X$ an abstract compact strictly pseudoconvex of dimension $\geq 5$, then $X$ satisfies condition (a), by a theorem of Boutet de Monvel [14]. Among embeddable strictly pseudoconvex CR manifolds of dimension three there are those carrying interesting geometric structures, such as transverse $S^{1}$ actions, cf. [9, 30, 53], conformal structures, cf. [7], or Sasakian structures, cf. [59].

The first author [44] showed that if the Levi form is non-degenerate and $\square_{b}^{(q)}$ has $L^{2}$ closed range for some $q \in\{0,1, \ldots, n-1\}$, then $\Pi^{(q)}(x, y)$ is a complex Fourier integral operator. Therefore the study of the singularities of the Szego" kernel is closely related to the closed range property of the Kohn Laplacian.

Kohn [52] proved that if $Y(q)$ fails but $Y(q-1)$ and $Y(q+1)$ hold on a compact CR manifold $X$, then $\square_{b}^{(q)}$ has $L^{2}$ closed range. In this case the result of [44] applies and we can describe the Szego" kernel $\Pi^{(q)}(x, y)$. On the negative side, Burns [19] showed that the closed range property fails in the case of the non-embeddable exemples of Grauert, Andreotti-Siu and Rossi [36, 1, 62]. 
Beside Kohn's criterion, it is very difficult to determine when $\square_{b}^{(q)}$ has $L^{2}$ closed range. Let's see a simple example. Let $[z]=\left[z_{1}, \ldots, z_{N}\right]$ be the homogeneous coordinates of $\mathbb{C P}^{N-1}$. Consider $1 \leq m \leq N-1$. Put

$$
X:=\left\{\left[z_{1}, \ldots, z_{N}\right] \in \mathbb{C P}^{N-1} ; \sum_{j=1}^{N} \lambda_{j}\left|z_{j}\right|=0\right\},
$$

where $\lambda_{j}<0$ for $1 \leq j \leq m$ and $\lambda_{j}>0$ for $m+1 \leq j \leq N$. Then, $X$ is a compact CR manifold of dimension $2(N-1)-1$ with CR structure $T^{1,0} X:=T^{1,0} \mathbb{C} \mathbb{P}^{N-1} \cap \mathbb{C} T X$. It is straightforward to see that the Levi form has exactly $m-1$ negative eigenvalues and $N-m-1$ positive eigenvalues at every point of $X$. Thus, when $q=m-1, N-m-1=q+1, Y(q)$ and $Y(q+1)$ fail and Kohn's criterion does not work in this case. Even in this simple example, it doesn't follow from Kohn's criterion that $\square_{b}^{(q)}$ has $L^{2}$ closed range. We are lead to ask the following questions:

Question 1.1. Let $X$ be compact CR manifold whose Levi form is non-degenerate of signature $\left(n_{-}, n_{+}\right)$. Assume $n_{+} \in\left\{n_{-}-1, n_{-}+1\right\}$. When does $\square_{b}^{(q)}$ have $L^{2}$ closed range for $q=n_{-}$? Note that in this case, $Y(q)$ and $Y(q+1)$ fail.

This question was asked by Boutet de Monvel. We will introduce another condition, called $W(q)$ (see Definition 6.22) which applied for CR manifolds with $S^{1}$ action shows that $\square_{b}^{(q)}$ has $L^{2}$ closed range in the situation of Question 1.1, see Theorem 6.23. In particular, the Kohn Laplacian on the manifold $X$ in the examples (1.1) has closed range.

Question 1.2. Let $X$ be a not-necessarily compact CR manifold and the Levi form can be degenerate (for example $X$ is weakly pseudoconvex). Assume that $\square_{b}^{(q)}$ has $L^{2}$ closed range. Does the Szegö kernel $\Pi^{(q)}(x, y)$ admit an asymptotic expansion on the set where the Levi form is non-degenerate?

Question 1.3. Find a natural local analytic condition (weaker than $L^{2}$ closed range condition) which implies that the Szegő kernel admits a local asymptotic expansion.

Without any regularity assumption, $\operatorname{Ker} \square_{b}^{(q)}$ could be trivial and therefore we consider the spectral projections $\Pi_{\leq \lambda}^{(q)}:=E([0, \lambda])$, for $\lambda>0$, where $E$ denotes the spectral measure of $\square_{b}^{(q)}$.

Question 1.4. Is $\Pi_{\leq \lambda}^{(q)}$ a Fourier integral operator, for every $\lambda>0$ ?

The purpose of this work is to answer Questions 1.1] 1.4. Our first main results tell us that on the non-degenerate part of the Levi form, $\Pi_{\leq \lambda}^{(q)}$ is a Fourier integral operator with complex phase, for every $\lambda>0$, and $\Pi_{\left(\lambda_{1}, \lambda_{2}\right]}^{(q)}:=E\left(\left(\lambda_{1}, \lambda_{2}\right]\right)$ is a smoothing operator, for every $0<\lambda_{1}<\lambda_{2}$.

Theorem 1.5. Let $X$ be a CR manifold whose Levi form is non-degenerate of constant signature $\left(n_{-}, n_{+}\right)$at each point of an open set $D \Subset X$. Then for every $\lambda>0$ the restriction of the spectral projector $\Pi_{\leq \lambda}^{(q)}$ to $D$ is a smoothing operator for $q \notin\left\{n_{-}, n_{+}\right\}$and is a Fourier integral operator with complex phase for $q \in\left\{n_{-}, n_{+}\right\}$. Moreover, in the latter case, the singularity of $\Pi_{\leq \lambda}^{(q)}$ does not depend on $\lambda$, in the sense that the difference $\Pi_{\leq \lambda_{1}}^{(q)}-\Pi_{\leq \lambda_{2}}^{(q)}$ is smoothing on $D$ for any $\lambda_{1}, \lambda_{2}>0$. 
The Fourier integral operators $A$ considered here (and in this paper) have kernels of the form

$$
A(x, y)=\int_{0}^{\infty} e^{i \varphi_{-}(x, y) t} s_{-}(x, y, t) d t+\int_{0}^{\infty} e^{i \varphi_{+}(x, y) t} s_{+}(x, y, t) d t+R(x, y),
$$

where the integrals are oscillatory integrals, $\varphi_{-}, \varphi_{+}$are complex phase functions, $s_{-}, s_{+}$ classical symbols of type $(1,0)$ and order $n-1, s_{-}=0$ if $q \neq n_{-}, s_{+}=0$ if $q \neq n_{+}$and $R$ a smooth function, see Section 2 for a precise definition.

A detailed version of Theorem 1.5 will be given in Theorems 4.1, 4.7 and 4.8. As a corollary of Theorem 1.5, we deduce:

Corollary 1.6. Let $X$ be a CR manifold of dimension $2 n-1$, whose Levi form is non-degenerate of constant signature at each point of an open set $D \Subset X$. Let $0 \leq q \leq n-1$ and $0<\lambda_{1}<\lambda_{2}$. Then, the projector $\Pi_{\left(\lambda_{1}, \lambda_{2}\right]}^{(q)}$ is a smoothing operator on $D$. In particular, if $X$ is compact and the Levi form is non-degenerate of constant signature on $X$, then the projector $\Pi_{\left(\lambda_{1}, \lambda_{2}\right]}^{(q)}$ is a smoothing operator on $X$.

As a consequence, we deduce that if $X$ is compact and the Levi form is non-degenerate of constant signature on $X$, then the spectrum of $\square_{b}^{(q)}$ in ]0, $\infty$ [ consists of point eigenvalues of finite multiplicity. Burns-Epstein [20, Theorem 1.3] proved that if $X$ is compact, strictly pseudoconvex of dimension three, then the spectrum of $\square_{b}^{(0)}$ in $] 0, \infty$ [ consists of point eigenvalues of finite multiplicity. We generalize their result to any $q \in\{0,1, \ldots, n-1\}$ and any dimension.

Theorem 1.7. We assume that $X$ is compact and the Levi form is non-degenerate of constant signature $\left(n_{-}, n_{+}\right)$on $X$. Fix $q \in\{0,1, \ldots, n-1\}$. Then, for any $\mu>0$, Spec $\square_{b}^{(q)} \cap[\mu, \infty[$ is a discrete subset of $\mathbb{R}$, any $\nu \in \operatorname{Spec} \square_{b}^{(q)}$ with $\nu>0$ is an eigenvalue of $\square_{b}^{(q)}$ and the eigenspace $H_{b, \nu}^{q}(X):=\left\{u \in \operatorname{Dom} \square_{b}^{(q)} ; \square_{b}^{(q)} u=\nu u\right\}$ is finite dimensional with $H_{b, \nu}^{q}(X) \subset \Omega^{0, q}(X)$.

If $\square_{b}^{(q)}$ has closed range, then $\Pi^{(q)}=\Pi_{\leq \lambda}^{(q)}$ for some $\lambda>0$, so we can deduce the asymptotic of the Szegő kernel from Theorem 1.5. We introduce now the following local and more flexible version of the closed range property.

Definition 1.8. Fix $q \in\{0,1,2, \ldots, n-1\}$. Let $Q: L_{(0, q)}^{2}(X) \rightarrow L_{(0, q)}^{2}(X)$ be a continuous operator. We say that $\square_{b}^{(q)}$ has local $L^{2}$ closed range on an open set $D \subset X$ with respect to $Q$ if for every $D^{\prime} \Subset D$, there exist constants $C_{D^{\prime}}>0$ and $p \in \mathbb{N}$, such that

$$
\left\|Q\left(I-\Pi^{(q)}\right) u\right\|^{2} \leq C_{D^{\prime}}\left(\left(\square_{b}^{(q)}\right)^{p} u \mid u\right), \quad \forall u \in \Omega_{0}^{0, q}\left(D^{\prime}\right) .
$$

When $D=X, Q$ is the identity map and $p=2$, this property is just the $L^{2}$ closed range property for $\square_{b}^{(q)}$. When $D=X, Q$ is the identity map, $p=1$ and $q=0$, this property is the $L^{2}$ closed range property for $\bar{\partial}_{b}$.

Theorem 1.9. Let $X$ be a CR manifold of dimension $2 n-1$, whose Levi form is non-degenerate of constant signature $\left(n_{-}, n_{+}\right)$at each point of an open set $D \Subset X$. Let $q \in\{0,1, \ldots, n-1\}$ and let $Q \in L_{\mathrm{cl}}^{0}\left(X, T^{* 0, q} X \otimes T^{* 0, q} X\right)$ be a classical pseudodifferential operator on $X$ and let $Q^{*} \in L_{\mathrm{cl}}^{0}\left(X, T^{* 0, q} X \otimes T^{* 0, q} X\right)$ be the $L^{2}$ adjoint of $Q$ with respect to $(\cdot \mid \cdot)$. Suppose that $\square_{b}^{(q)}$ has local $L^{2}$ closed range on $D$ with respect to $Q$ and $Q \Pi^{(q)}=\Pi^{(q)} Q$ on $L_{(0, q)}^{2}(X)$. Then, $Q^{*} \Pi^{(q)} Q$ is smoothing on $D$ if $q \notin\left\{n_{-}, n_{+}\right\}$and is a Fourier integral operator with complex phase if $q \in\left\{n_{-}, n_{+}\right\}$. 
This result will be proved in Section 5, see Theorem 5.1 for a detailed version of Theorem 1.9.

For $Q \in L_{\mathrm{cl}}^{m}(X)$, let $\sigma_{Q}(x, \xi) \in \mathscr{C}^{\infty}\left(T^{*} M\right)$ denote the principal symbol of $Q$. Let $\Sigma=\Sigma^{-} \cup \Sigma^{+}$ be the characteristic manifold of $\square_{b}^{(q)}$ (see (2.13) ). By using Theorem 1.9, we establish the following local embedding theorem (see Section 5 for a proof).

Theorem 1.10. Let $X$ be a CR manifold of dimension $2 n-1$, whose Levi form is positive at each point of an open set $D \Subset X$. Let $Q \in L_{\mathrm{cl}}^{0}(X)$ with $Q \Pi^{(0)}=\Pi^{(0)} Q$ and $\sigma_{Q}(x, \xi) \neq 0$ at each point of $\Sigma^{-}$. Suppose that $\square_{b}^{(0)}$ has local $L^{2}$ closed range on $D$ with respect to $Q$. Then, for any point $x_{0} \in D$, there is an open neighborhood $\hat{D} \Subset D$ of $x_{0}$ such that $\hat{D}$ can be embedded into $\mathbb{C}^{n}$ by $a$ global CR map.

We notice that in Theorem 1.10, $\square_{b}^{(0)}$ might not have $L^{2}$ closed range, however, with the help of the operator $Q$, we can still understand the Szegö projection and produce many global CR functions.

We will apply Theorem 1.9 to establish Szegő kernel asymptotic expansions on compact CR manifolds with transversal CR $S^{1}$ actions under certain Levi curvature assumptions.

Theorem 1.11. Let $\left(X, T^{1,0} X\right)$ be a compact $C R$ manifold of dimension $2 n-1, n \geq 2$, with a transversal $C R S^{1}$ action and let $T \in \mathscr{C}^{\infty}(X, T X)$ be the real vector field induced by this $S^{1}$ action. For $m \in \mathbb{Z}$, let $\mathcal{B}_{m}^{0, q}(X) \subset L_{(0, q)}^{2}(X)$ be the completion of

$$
B_{m}^{0, q}(X):=\left\{u \in \Omega^{0, q}(X) ; T u=-\sqrt{-1} m u\right\}
$$

and let $Q_{\leq 0}^{(q)}: L_{(0, q)}^{2}(X) \rightarrow \oplus_{m \in \mathbb{Z}, m \leq 0} \mathcal{B}_{m}^{0, q}(X)$ be the orthogonal projection. Assume that $Z(q)$ fails but $Z(q-1)$ and $Z(q+1)$ hold at every point of $X$. Then, $\square_{b}^{(q)}$ has local $L^{2}$ closed range on $X$ with respect to $Q_{\leq 0}^{(q)}$. Suppose further that the Levi form is non-degenerate of constant signature $\left(n_{-}, n_{+}\right)$on an open canonical coordinate patch $D \Subset X$. Then, $Q_{\leq 0}^{(q)} \Pi^{(q)} Q_{\leq 0}^{(q)}$ is smoothing on $D$ if $q \neq n_{-}$and $Q_{\leq 0}^{(q)} \Pi^{(q)} Q_{\leq 0}^{(q)}$ is a Fourier integral operator with complex phase if $q=n_{-}$.

This result will be proved in $\S 6$, see Theorem 6.20 for the details and see Definition 6.1 and Definition 6.7 for the meanings of transversal CR $S^{1}$ action and condition $Z(q)$. As a consequence we obtain (cf. Theorem 6.23 and Corollary 6.24):

Theorem 1.12. Let $\left(X, T^{1,0} X\right)$ be a compact $C R$ manifold of dimension $2 n-1, n \geq 2$, with a transversal CR $S^{1}$ action. Assume $W(q)$ holds on $X$ for some $q \in\{0,1, \ldots, n-1\}$. Then $\square_{b}^{(q)}$ has $L^{2}$ closed range. In particular, for any CR submanifold in $\mathbb{C P}^{N}$ of the form (1.1), the associated Szegő kernel $\Pi^{(q)}(x, y)$ admits a full asymptotic expansion.

We notice that if the Levi form is non-degenerate of constant signature on $X$ then $W(q)$ holds on $X$ (see Definition 6.22). In particular, for a 3-dimensional compact strictly pseudoconvex CR manifold, $W(0)$ holds on $X$. Hence, $\square_{b}^{(0)}$ has $L^{2}$ closed range if $X$ admits a transversal CR $S^{1}$ action. From this, we deduce the following global embeddablity of Lempert [53, Theorem 2.1], cf. also [30, Theorem A16] (see Section 6).

Theorem 1.13. Let $\left(X, T^{1,0} X\right)$ be a compact strictly pseudoconvex $C R$ manifold of dimension three with a transversal $C R S^{1}$ action. Then $X$ can be $C R$ embedded into $\mathbb{C}^{N}$, for some $N \in \mathbb{N}$.

Note that Baouendi-Rothschild-Treves [3] proved that the existence of a local transverse CR action implies local embeddability. Let us point out that transversality in Theorem 1.13 
cannot be dispensed with: the non-embeddable example of Grauert, Andreotti-Siu and Rossi [36, 1, 62] admits a nontransversal circle action; see also the example of of Barrett [4] which admits a transverse CR torus action, but no one-dimensional sub-action exists which itself is transverse. The embeddable small deformations of of $S^{1}$ invariant strictly pseudoconvex CR structures on circle bundles over Riemann surfaces were described by Epstein [30].

Theorem 1.9 yields immediately the following.

Theorem 1.14. Suppose that $X$ is a CR manifold such that $\square_{b}^{(0)}$ has closed range in $L^{2}$. Then the Szegó projector $\Pi^{(0)}$ is a Fourier integral operator on the subset where the Levi form is positive definite.

Corollary 1.15. Let $X$ be a compact pseudoconvex CR manifold satisfying one of the following conditions:

(i) $X=\partial M$, where $M$ is a relatively compact pseudoconvex domain in a complex manifold, such that there exists a strictly psh function in a neighborhood of $X$.

(ii) $X$ admits a CR embedding into some Euclidean space $\mathbb{C}^{N}$.

Then the Szegó projector $\Pi^{(0)}$ is a Fourier integral operator on the subset where the Levi form is positive definite.

Indeed, it was shown that $\bar{\partial}_{b}$ has closed range in $L^{2}$ under condition (i) in [52, p. 543] and under condition (ii) in [2, 61]. For boundaries of pseudoconvex domains in $\mathbb{C}^{n}$ the closed range property was shown in $[10,[52,63]$. Note also that any three-dimensional pseudoconvex and of finite type CR manifold $X$ admits a CR embedding into some $\mathbb{C}^{N}$ if $\bar{\partial}_{b}$ has $L^{2}$ closed range, cf. [24].

We can give a very concrete description of the Szegő kernel in case (i) of Corollary 1.15. Let $M$ be a relatively compact domain with smooth boundary in a complex manifold $M^{\prime}$ and $M=\{\rho<0\}$ where $\rho \in \mathscr{C}^{\infty}\left(M^{\prime}\right)$ is a defining function of $M$. We assume that the Levi form $\mathcal{L}(\rho)$ is everywhere positive semi-definite on the complex tangent space to $X=\partial M$ and is positive definite of a subset $D \subset X$. Fix $D_{0} \Subset D$ and let $U$ be a small neighbourhood of $D_{0}$ in $M^{\prime}$. As in [17], one can construct an almost-analytic extension $\varphi=\varphi(x, y): M^{\prime} \times M^{\prime} \rightarrow \mathbb{C}$ of $\rho$ with the following properties:

$$
\begin{aligned}
& \varphi(x, x)=\frac{1}{\sqrt{-1}} \varrho(x) \text { and } \partial_{y} \varphi, \bar{\partial}_{x} \varphi \text { vanish to infinite order on the diagonal } x=y . \\
& \varphi(y, x)=\frac{\varphi(x, y)}{\operatorname{Im} \varphi(x, y) \geq c|x-y|^{2} \text { on } U \times U, \text { where } c>0 \text { is a constant. }}
\end{aligned}
$$

Then on $D_{0}$, the Szegö kernel $\Pi^{(0)}(x, y)$ of $X$ has the form

$$
\begin{aligned}
\Pi^{(0)}(x, y) & =\int_{0}^{\infty} e^{i \varphi(x, y) t} s(x, y, t) d t+R(x, y) \\
& =F(x, y)(-i \varphi(x, y)+0)^{-n}+G(x, y) \log (-i \varphi(x, y)+0)
\end{aligned}
$$

for some smooth functions $F, G$ and $R$. Here we denote by $(-i \varphi(x, y)+0)^{-n}, \log (-i \varphi(x, y)+0)$ the distributions limit of $(-i \varphi(x, y)+\varepsilon)^{-n}$ and $\log (-i \varphi(x, y)+\varepsilon)$ as $\varepsilon \rightarrow 0+$. See Section 7 for more details.

Our method can be extended to non-compact weakly pseudoconvex tube domains in $\mathbb{C}^{n}$ with basis a strictly pseudoconvex domain in $\mathbb{C}^{n-1}$ cf. Theorems 7.4 and 7.5 . 
Let us finally mention that the analysis of the Szegő kernels was also used to study embeddings given by CR sections of a positive CR bundle, introduced in [47] (see also [45, 46]).

The Szegő projector plays an important role in embedding problems also through the framework of relative index for Szegő projectors introduced by Epstein cf. [31]. One outcome of this analysis is the solution of the relative index conjecture [32], which implies that the set of embeddable deformations of a strictly pseudoconvex CR structure on a compact three-dimensional manifold is closed in the $\mathscr{C}^{\infty}$-topology.

The layout of this paper is as follows. In Section 2, we collect some notations, definitions and statements we use throughout.

In Section 3, we review some results in [44] about the existence of a microlocal Hodge decomposition of the Kohn Laplacian on an open set of a CR manifold where the Levi form is non-degenerate.

In Section 4, we first study the microlocal behahaviour of the spectral function and by using the microlocal Hodge decomposition of the Kohn Laplacian established in [44], we prove Theorem 1.5. Furthermore, by using Theorem 1.5 and some standard technique in functional analysis, we prove Theorem 1.7 .

Section 5 is devoted to proving Theorem 1.9 and 1.10 .

In Section 6, we study CR manifolds with transversal CR $S^{1}$ actions. We introduce the microlocal cut-off functions $Q_{<0}^{(q)}$ and $Q_{>0}^{(q)}$ and study the closed range property with respect to these operators. Finally we establish Theorems 1.11, 1.12 and 1.13.

In Section 7, by using Hörmander's $L^{2}$ estimates, we establish the local $L^{2}$ closed range property for $\square_{b}^{(0)}$ with respect to $Q^{(0)}$ for some weakly pseudocnovex tube domains in $\mathbb{C}^{n}$, hence establish the asymptotics of the Szego" kernel (see Theorem 7.4 and Theorem 7.5).

Finally, in Section 8, we prove the technical Theorem 5.4 by using semi-classical analysis and global theory of complex Fourier integral operators of Melin-Sjöstrand [60]. Theorem 5.4 will be used in the proof of Theorem 1.9 .

\section{Preliminaries}

2.1. Standard notations. We shall use the following notations: $\mathbb{N}=\{1,2, \ldots\}, \mathbb{N}_{0}=\mathbb{N} \cup\{0\}$, $\mathbb{R}$ is the set of real numbers, $\overline{\mathbb{R}}_{+}:=\{x \in \mathbb{R} ; x \geq 0\}$. For a multiindex $\alpha=\left(\alpha_{1}, \ldots, \alpha_{n}\right) \in \mathbb{N}_{0}^{n}$ we denote by $|\alpha|=\alpha_{1}+\ldots+\alpha_{n}$ its norm and by $l(\alpha)=n$ its length. For $m \in \mathbb{N}$, write $\alpha \in\{1, \ldots, m\}^{n}$ if $\alpha_{j} \in\{1, \ldots, m\}, j=1, \ldots, n$. $\alpha$ is strictly increasing if $\alpha_{1}<\alpha_{2}<\ldots<\alpha_{n}$. For $x=\left(x_{1}, \ldots, x_{n}\right)$ we write

$$
\begin{aligned}
& x^{\alpha}=x_{1}^{\alpha_{1}} \ldots x_{n}^{\alpha_{n}} \\
& \partial_{x_{j}}=\frac{\partial}{\partial x_{j}}, \quad \partial_{x}^{\alpha}=\partial_{x_{1}}^{\alpha_{1}} \ldots \partial_{x_{n}}^{\alpha_{n}}=\frac{\partial^{|\alpha|}}{\partial x^{\alpha}}, \\
& D_{x_{j}}=\frac{1}{i} \partial_{x_{j}}, \quad D_{x}^{\alpha}=D_{x_{1}}^{\alpha_{1}} \ldots D_{x_{n}}^{\alpha_{n}}, \quad D_{x}=\frac{1}{i} \partial_{x} .
\end{aligned}
$$

Let $z=\left(z_{1}, \ldots, z_{n}\right), z_{j}=x_{2 j-1}+i x_{2 j}, j=1, \ldots, n$, be coordinates of $\mathbb{C}^{n}$. We write

$$
\begin{aligned}
& z^{\alpha}=z_{1}^{\alpha_{1}} \ldots z_{n}^{\alpha_{n}}, \quad \bar{z}^{\alpha}=\bar{z}_{1}^{\alpha_{1}} \ldots \bar{z}_{n}^{\alpha_{n}}, \\
& \partial_{z_{j}}=\frac{\partial}{\partial z_{j}}=\frac{1}{2}\left(\frac{\partial}{\partial x_{2 j-1}}-i \frac{\partial}{\partial x_{2 j}}\right), \quad \partial_{\bar{z}_{j}}=\frac{\partial}{\partial \bar{z}_{j}}=\frac{1}{2}\left(\frac{\partial}{\partial x_{2 j-1}}+i \frac{\partial}{\partial x_{2 j}}\right), \\
& \partial_{z}^{\alpha}=\partial_{z_{1}}^{\alpha_{1}} \ldots \partial_{z_{n}}^{\alpha_{n}}=\frac{\partial^{|\alpha|}}{\partial z^{\alpha}}, \quad \partial_{\bar{z}}^{\alpha}=\partial_{\bar{z}_{1}}^{\alpha_{1}} \ldots \partial_{\bar{z}_{n}}^{\alpha_{n}}=\frac{\partial^{|\alpha|}}{\partial \bar{z}^{\alpha}} .
\end{aligned}
$$


For $j, s \in \mathbb{Z}$, set $\delta_{j, s}=1$ if $j=s, \delta_{j, s}=0$ if $j \neq s$.

Let $M$ be a $\mathscr{C}^{\infty}$ paracompact manifold. We let $T M$ and $T^{*} M$ denote the tangent bundle of $M$ and the cotangent bundle of $M$ respectively. The complexified tangent bundle of $M$ and the complexified cotangent bundle of $M$ are be denoted by $\mathbb{C} T M$ and $\mathbb{C} T^{*} M$, respectively. Write $\langle\cdot, \cdot\rangle$ to denote the pointwise duality between $T M$ and $T^{*} M$. We extend $\langle\cdot, \cdot\rangle$ bilinearly to $\mathbb{C} T M \times \mathbb{C} T^{*} M$. Let $G$ be a $\mathscr{C}^{\infty}$ vector bundle over $M$. The fiber of $G$ at $x \in M$ will be denoted by $G_{x}$. Let $E$ be another vector bundle over $M$. We write $G \otimes E$ to denote the vector bundle over $M \times M$ with fiber over $(x, y) \in M \times M$ consisting of the linear maps from $G_{x}$ to $E_{y}$. Let $Y \subset M$ be an open set. From now on, the spaces of distribution sections of $G$ over $Y$ and smooth sections of $G$ over $Y$ will be denoted by $\mathscr{D}^{\prime}(Y, G)$ and $\mathscr{C}^{\infty}(Y, G)$ respectively. Let $\mathscr{E}^{\prime}(Y, G)$ be the subspace of $\mathscr{D}^{\prime}(Y, G)$ whose elements have compact support in $Y$. For $m \in \mathbb{R}$, let $H^{m}(Y, G)$ denote the Sobolev space of order $m$ of sections of $G$ over $Y$. Put

$$
\begin{gathered}
H_{\mathrm{loc}}^{m}(Y, G)=\left\{u \in \mathscr{D}^{\prime}(Y, G) ; \varphi u \in H^{m}(Y, G), \forall \varphi \in \mathscr{C}_{0}^{\infty}(Y)\right\}, \\
H_{\mathrm{comp}}^{m}(Y, G)=H_{\mathrm{loc}}^{m}(Y, G) \cap \mathscr{E}^{\prime}(Y, G) .
\end{gathered}
$$

We recall the Schwartz kernel theorem [42, Theorems 5.2.1, 5.2.6], [57, Thorem B.2.7], [65, p. 296]. Let $G$ and $E$ be $\mathscr{C}^{\infty}$ vector bundles over a paracompact orientable $\mathscr{C}^{\infty}$ manifold $M$ equipped with a smooth density of integration. If $A: \mathscr{C}_{0}^{\infty}(M, G) \rightarrow \mathscr{D}^{\prime}(M, E)$ is continuous, we write $K_{A}(x, y)$ or $A(x, y)$ to denote the distribution kernel of $A$. The following two statements are equivalent

(a) $A$ is continuous: $\mathscr{E}^{\prime}(M, G) \rightarrow \mathscr{C}^{\infty}(M, E)$,

(b) $K_{A} \in \mathscr{C}^{\infty}\left(M \times M, G_{y} \otimes E_{x}\right)$.

If $A$ satisfies (a) or (b), we say that $A$ is smoothing. Let $A, B: \mathscr{C}_{0}^{\infty}(M, G) \rightarrow \mathscr{D}^{\prime}(M, E)$ be continuous operators. We write $A \equiv B$ (on $M$ ) if $A-B$ is a smoothing operator.

We say that $A$ is properly supported if the restrictions of the two projections $(x, y) \mapsto x$, $(x, y) \mapsto y$ to $\operatorname{Supp} K_{A}$ are proper.

Let $H(x, y) \in \mathscr{D}^{\prime}\left(M \times M, G_{y} \otimes E_{x}\right)$. We write $H$ to denote the unique continuous operator $\mathscr{C}_{0}^{\infty}(M, G) \rightarrow \mathscr{D}^{\prime}(M, E)$ with distribution kernel $H(x, y)$. In this work, we identify $H$ with $H(x, y)$.

2.2. Set up and Terminology. Let $\left(X, T^{1,0} X\right)$ be an orientable not necessarily compact, paracompact CR manifold of dimension $2 n-1, n \geqslant 2$, where $T^{1,0} X$ is a CR structure of $X$. Recall that $T^{1,0} X$ is a complex $n-1$ dimensional subbundle of $\mathbb{C} T X$, satisfying $T^{1,0} X \cap T^{0,1} X=\{0\}$, where $T^{0,1} X=\overline{T^{1,0} X}$, and $[\mathcal{V}, \mathcal{V}] \subset \mathcal{V}$, where $\mathcal{V}=\mathscr{C}^{\infty}\left(X, T^{1,0} X\right)$.

Fix a smooth Hermitian metric $\langle\cdot \mid \cdot\rangle$ on $\mathbb{C} T X$ so that $\langle u \mid v\rangle$ is real if $u$, $v$ are real tangent vectors and $T^{1,0} X$ is orthogonal to $T^{0,1} X:=\overline{T^{1,0} X}$. Then locally there is a real vector field $T$ of length one which is pointwise orthogonal to $T^{1,0} X \oplus T^{0,1} X$. $T$ is unique up to the choice of sign. For $v \in \mathbb{C} T X$, we write $|v|^{2}:=\langle v \mid v\rangle$. We denote by $T^{* 1,0} X$ and $T^{* 0,1} X$ the dual bundles of $T^{1,0} X$ and $T^{0,1} X$, respectively. Define the vector bundle of $(0, q)$ forms by $T^{* 0, q} X:=\Lambda^{q} T^{* 0,1} X$. The Hermitian metric $\langle\cdot \mid \cdot\rangle$ on $\mathbb{C} T X$ induces, by duality, a Hermitian metric on $\mathbb{C} T^{*} X$ and also on the bundles of $(0, q)$ forms $T^{* 0, q} X, q=0,1, \ldots, n-1$. We shall also denote all these induced metrics by $\langle\cdot \mid \cdot\rangle$. For $u \in T^{* 0, q} X$, we write $|u|^{2}:=\langle u \mid u\rangle$. Let $D \subset X$ be an open set. Let $\Omega^{0, q}(D)$ denote the space of smooth sections of $T^{* 0, q} X$ over $D$ and let $\Omega_{0}^{0, q}(D)$ be the subspace of $\Omega^{0, q}(D)$ whose elements have compact support in $D$. 
Locally there exists an orthonormal frame $\omega_{1}, \ldots, \omega_{n-1}$ of the bundle $T^{* 1,0} X$. The real $(2 n-2)$ form $\omega=i^{n-1} \omega_{1} \wedge \bar{\omega}_{1} \wedge \ldots \wedge \omega_{n-1} \wedge \bar{\omega}_{n-1}$ is independent of the choice of the orthonormal frame. Thus $\omega$ is globally defined. Locally there exists a real 1-form $\omega_{0}$ of length one which is orthogonal to $T^{* 1,0} X \oplus T^{* 0,1} X$. The form $\omega_{0}$ is unique up to the choice of sign. Since $X$ is orientable, there is a nowhere vanishing $(2 n-1)$ form $\Theta$ on $X$. Thus, $\omega_{0}$ can be specified uniquely by requiring that $\omega \wedge \omega_{0}=f \Theta$, where $f$ is a positive function. Therefore $\omega_{0}$, so chosen, is globally defined. We call $\omega_{0}$ the uniquely determined global real 1-form. We take a vector field $T$ so that

$$
|T|=1, \quad\left\langle T, \omega_{0}\right\rangle=-1 .
$$

Therefore $T$ is uniquely determined. We call $T$ the uniquely determined global real vector field. We have the pointwise orthogonal decompositions:

$$
\mathbb{C} T^{*} X=T^{* 1,0} X \oplus T^{* 0,1} X \oplus\left\{\lambda \omega_{0} ; \lambda \in \mathbb{C}\right\}, \mathbb{C} T X=T^{1,0} X \oplus T^{0,1} X \oplus\{\lambda T ; \lambda \in \mathbb{C}\} .
$$

Definition 2.1. For $p \in X$, the Levi form $\mathcal{L}_{p}$ is the Hermitian quadratic form on $T_{p}^{1,0} X$ defined as follows. For any $Z, W \in T_{p}^{1,0} X$, pick $\mathcal{Z}, \mathcal{W} \in \mathscr{C}^{\infty}\left(X, T^{1,0} X\right)$ such that $\mathcal{Z}(p)=Z, \mathcal{W}(p)=$ $W$. Set

$$
\mathcal{L}_{p}(Z, \bar{W})=\frac{1}{2 i}\left\langle[\mathcal{Z}, \overline{\mathcal{W}}](p), \omega_{0}(p)\right\rangle,
$$

where $[\mathcal{Z}, \overline{\mathcal{W}}]=\mathcal{Z} \overline{\mathcal{W}}-\overline{\mathcal{W}} \mathcal{Z}$ denotes the commutator of $\mathcal{Z}$ and $\overline{\mathcal{W}}$. Note that $\mathcal{L}_{p}$ does not depend of the choices of $\mathcal{Z}$ and $\mathcal{W}$.

Locally there exists an orthonormal basis $\left\{\mathcal{Z}_{1}, \ldots, \mathcal{Z}_{n-1}\right\}$ of $T^{1,0} X$ with respect to the Hermitian metric $\langle\cdot \mid \cdot\rangle$ such that $\mathcal{L}_{p}$ is diagonal in this basis, $\mathcal{L}_{p}\left(\mathcal{Z}_{j}, \overline{\mathcal{Z}}_{l}\right)=\delta_{j, l} \lambda_{j}(p)$. The entries $\lambda_{1}(p), \ldots, \lambda_{n-1}(p)$ are called the eigenvalues of the Levi form at $p \in X$ with respect to $\langle\cdot \mid \cdot\rangle$.

Definition 2.2. Given $q \in\{0, \ldots, n-1\}$, the Levi form is said to satisfy condition $Y(q)$ at $p \in X$, if $\mathcal{L}_{p}$ has at least either $\min (q+1, n-q)$ pairs of eigenvalues with opposite signs or $\max (q+1, n-q)$ eigenvalues of the same sign. Notice that the sign of the eigenvalues does not depend on the choice of the metric $\langle\cdot \mid \cdot\rangle$.

Let

$$
\bar{\partial}_{b}: \Omega^{0, q}(X) \rightarrow \Omega^{0, q+1}(X)
$$

be the tangential Cauchy-Riemann operator. We will work with two volume forms on $X$ :

- A given smooth positive $(2 n-1)$-form $m(x)$ on $X$.

- The volume form $v(x)$ induced by the Hermitian metric $\langle\cdot \mid \cdot\rangle$.

The natural global $L^{2}$ inner product $(\cdot \mid \cdot)$ on $\Omega_{0}^{0, q}(X)$ induced by $m(x)$ and $\langle\cdot \mid \cdot\rangle$ is given by

$$
(u \mid v):=\int_{X}\langle u(x) \mid v(x)\rangle m(x), \quad u, v \in \Omega_{0}^{0, q}(X) .
$$

We denote by $L_{(0, q)}^{2}(X)$ the completion of $\Omega_{0}^{0, q}(X)$ with respect to $(\cdot \mid \cdot)$. We write $L^{2}(X):=$ $L_{(0,0)}^{2}(X)$. We extend $(\cdot \mid \cdot)$ to $L_{(0, q)}^{2}(X)$ in the standard way. For $f \in L_{(0, q)}^{2}(X)$, we denote $\|f\|^{2}:=(f \mid f)$. We extend $\bar{\partial}_{b}$ to $L_{(0, r)}^{2}(X), r=0,1, \ldots, n-1$, by

$$
\bar{\partial}_{b}: \operatorname{Dom} \bar{\partial}_{b} \subset L_{(0, r)}^{2}(X) \rightarrow L_{(0, r+1)}^{2}(X),
$$


where $\operatorname{Dom} \bar{\partial}_{b}:=\left\{u \in L_{(0, r)}^{2}(X) ; \bar{\partial}_{b} u \in L_{(0, r+1)}^{2}(X)\right\}$, where for any $u \in L_{(0, r)}^{2}(X), \bar{\partial}_{b} u$ is defined in the sense of distributions. We also write

$$
\bar{\partial}_{b}^{*}: \operatorname{Dom} \bar{\partial}_{b}^{*} \subset L_{(0, r+1)}^{2}(X) \rightarrow L_{(0, r)}^{2}(X)
$$

to denote the Hilbert space adjoint of $\bar{\partial}_{b}$ in the $L^{2}$ space with respect to $(\cdot \mid \cdot)$. Let $\square_{b}^{(q)}$ denote the (Gaffney extension) of the Kohn Laplacian given by

$$
\begin{gathered}
\operatorname{Dom} \square_{b}^{(q)}=\left\{s \in L_{(0, q)}^{2}(X) ; s \in \operatorname{Dom} \bar{\partial}_{b} \cap \operatorname{Dom} \bar{\partial}_{b}^{*}, \bar{\partial}_{b} s \in \operatorname{Dom} \bar{\partial}_{b}^{*}, \bar{\partial}_{b}^{*} s \in \operatorname{Dom} \bar{\partial}_{b}\right\}, \\
\square_{b}^{(q)} s=\bar{\partial}_{b} \bar{\partial}_{b}^{*} s+\bar{\partial}_{b}^{*} \bar{\partial}_{b} s \text { for } s \in \operatorname{Dom} \square_{b}^{(q)} .
\end{gathered}
$$

By a result of Gaffney, for every $q=0,1, \ldots, n-1, \square_{b}^{(q)}$ is a positive self-adjoint operator (see [57, Proposition 3.1.2]). That is, $\square_{b}^{(q)}$ is self-adjoint and the spectrum of $\square_{b}^{(q)}$ is contained in $\overline{\mathbb{R}}_{+}, q=0,1, \ldots, n-1$. We shall write Spec $\square_{b}^{(q)}$ to denote the spectrum of $\square_{b}^{(q)}$. For a Borel set $B \subset \mathbb{R}$ we denote by $E(B)$ the spectral projection of $\square_{b}^{(q)}$ corresponding to the set $B$, where $E$ is the spectral measure of $\square_{b}^{(q)}$ (see Davies [25, §2] for the precise meanings of spectral projection and spectral measure). For $\lambda_{1}>\lambda \geq 0$, we set

$$
\begin{aligned}
& H_{b, \leq \lambda}^{q}(X):=\operatorname{Ran} E((-\infty, \lambda]) \subset L_{(0, q)}^{2}(X), \\
& H_{b,>\lambda}^{q}(X):=\operatorname{Ran} E((\lambda, \infty)) \subset L_{(0, q)}^{2}(X), \\
& H_{b,\left(\lambda, \lambda_{1}\right]}^{q}(X):=\operatorname{Ran} E\left(\left(\lambda, \lambda_{1}\right]\right) \subset L_{(0, q)}^{2}(X) .
\end{aligned}
$$

For $\lambda=0$, we denote

$$
H_{b}^{q}(X):=H_{b, \leq 0}^{q}(X)=\operatorname{Ker} \square_{b}^{(q)}
$$

For $\lambda_{1}>\lambda \geq 0$, let

$$
\begin{aligned}
& \Pi_{\leq \lambda}^{(q)}: L_{(0, q)}^{2}(X) \rightarrow H_{b, \leq \lambda}^{q}(X), \\
& \Pi_{>\lambda}^{(q)}: L_{(0, q)}^{2}(X) \rightarrow H_{b,>\lambda}^{q}(X), \\
& \Pi_{\left(\lambda, \lambda_{1}\right]}^{(q)}: L_{(0, q)}^{2}(X) \rightarrow H_{b,\left(\lambda, \lambda_{1}\right]}^{q}(X)
\end{aligned}
$$

be the orthogonal projections with respect to the product $(\cdot \mid \cdot)$ defined in $(2.5)$ and let

$$
\Pi_{\leq \lambda}^{(q)}(x, y), \Pi_{>\lambda}^{(q)}(x, y), \Pi_{\left(\lambda, \lambda_{1}\right]}^{(q)}(x, y) \in \mathscr{D}^{\prime}\left(X \times X, T_{y}^{* 0, q} X \otimes T_{x}^{* 0, q} X\right),
$$

denote the distribution kernels of $\Pi_{\leq \lambda}^{(q)}, \Pi_{>\lambda}^{(q)}$ and $\Pi_{\left(\lambda, \lambda_{1}\right]}^{(q)}$, respectively. For $\lambda=0$, we denote $\Pi^{(q)}:=\Pi_{\leq 0}^{(q)}, \Pi^{(q)}(x, y):=\Pi_{\leq 0}^{(q)}(x, y)$.

We recall now some notions of microlocal analysis. The characteristic manifold of $\square_{b}^{(q)}$ is given by $\Sigma=\Sigma^{+} \cup \Sigma^{-}$, where

$$
\Sigma^{+}=\left\{\left(x, \lambda \omega_{0}(x)\right) \in T^{*} X ; \lambda>0\right\}, \Sigma^{-}=\left\{\left(x, \lambda \omega_{0}(x)\right) \in T^{*} X ; \lambda<0\right\},
$$

where $\omega_{0} \in \mathscr{C}^{\infty}\left(X, T^{*} X\right)$ is the uniquely determined global 1-form (see the discussion before (2.1)).

Let $\Gamma$ be a conic open set of $\mathbb{R}^{M}, M \in \mathbb{N}$, and let $E$ be a smooth vector bundle over $\Gamma$. Let $m \in \mathbb{R}, 0 \leq \rho, \delta \leq 1$. Let $S_{\rho, \delta}^{m}(\Gamma, E)$ denote the Hörmander symbol space on $\Gamma$ with values in $E$ of order $m$ type $(\rho, \delta)$ and let $S_{\mathrm{cl}}^{m}(\Gamma, E)$ denote the space of classical symbols on $\Gamma$ with values in $E$ of order $m$, see Grigis-Sjöstrand [37, Definition 1.1 and p. 35] and Definition 2.3 below. 
Let $D$ be an open set of $X$. Let $L_{\frac{1}{2}, \frac{1}{2}}^{m}\left(D, T^{* 0, q} X \otimes T^{* 0, q} X\right)$ and $L_{\mathrm{cl}}^{m}\left(D, T^{* 0, q} D \otimes T^{* 0, q} D\right)$ denote the space of pseudodifferential operators on $D$ of order $m$ type $\left(\frac{1}{2}, \frac{1}{2}\right)$ from sections of $T^{* 0, q} X$ to sections of $T^{* 0, q} X$ and the space of classical pseudodifferential operators on $D$ of order $m$ from sections of $T^{* 0, q} X$ to sections of $T^{* 0, q} X$ respectively. The classical result of Calderon and Vaillancourt tells us that for any $A \in L_{\frac{1}{2}, \frac{1}{2}}^{m}\left(D, T^{* 0, q} X \otimes T^{* 0, q} X\right)$,

$$
A: H_{\text {comp }}^{s}\left(D, T^{* 0, q} X\right) \rightarrow H_{\text {loc }}^{s-m}\left(D, T^{* 0, q} X\right) \text { is continuous, for every } s \in \mathbb{R} .
$$

We refer to Hörmander [43] for a proof.

Definition 2.3. For $m \in \mathbb{R}, S_{1,0}^{m}\left(D \times D \times \mathbb{R}_{+}, T_{y}^{* 0, q} X \otimes T_{x}^{* 0, q} X\right)$ is the space of all $a(x, y, t) \in$ $\mathscr{C}^{\infty}\left(D \times D \times \mathbb{R}_{+}, T_{y}^{* 0, q} X \otimes T_{x}^{* 0, q} X\right)$ such that for all compact $K \Subset D \times D$ and all $\alpha, \beta \in \mathbb{N}_{0}^{2 n-1}$, $\gamma \in \mathbb{N}_{0}$, there is a constant $C_{\alpha, \beta, \gamma}>0$ such that

$$
\left|\partial_{x}^{\alpha} \partial_{y}^{\beta} \partial_{t}^{\gamma} a(x, y, t)\right| \leq C_{\alpha, \beta, \gamma}(1+|t|)^{m-|\gamma|}, \quad \forall(x, y, t) \in K \times \mathbb{R}_{+}, \quad t \geq 1 .
$$

Put

$$
S^{-\infty}\left(D \times D \times \mathbb{R}_{+}, T_{y}^{* 0, q} X \otimes T_{x}^{* 0, q} X\right):=\bigcap_{m \in \mathbb{R}} S_{1,0}^{m}\left(D \times D \times \mathbb{R}_{+}, T_{y}^{* 0, q} X \otimes T_{x}^{* 0, q} X\right) .
$$

Let $a_{j} \in S_{1,0}^{m_{j}}\left(D \times D \times \mathbb{R}_{+}, T_{y}^{* 0, q} X \otimes T_{x}^{* 0, q} X\right), j=0,1,2, \ldots$ with $m_{j} \rightarrow-\infty, j \rightarrow \infty$. Then there exists $a \in S_{1,0}^{m_{0}}\left(D \times D \times \mathbb{R}_{+}, T_{y}^{* 0, q} X \otimes T_{x}^{* 0, q} X\right)$ unique modulo $S^{-\infty}$, such that $a-\sum_{j=0}^{k-1} a_{j} \in$ $S_{1,0}^{m_{k}}\left(D \times D \times \mathbb{R}_{+}, T_{y}^{* 0, q} X \otimes T_{x}^{* 0, q} X\right)$ for $k=0,1,2, \ldots$

If $a$ and $a_{j}$ have the properties above, we write $a \sim \sum_{j=0}^{\infty} a_{j}$ in $S_{1,0}^{m_{0}}\left(D \times D \times \mathbb{R}_{+}, T_{y}^{* 0, q} X \otimes\right.$ $\left.T_{x}^{* 0, q} X\right)$. We write

$$
s(x, y, t) \in S_{\mathrm{cl}}^{n-1}\left(D \times D \times \mathbb{R}_{+}, T_{y}^{* 0, q} X \otimes T_{x}^{* 0, q} X\right)
$$

if $s(x, y, t) \in S_{1,0}^{n-1}\left(D \times D \times \mathbb{R}_{+}, T_{y}^{* 0, q} X \otimes T_{x}^{* 0, q} X\right)$ and

$$
\begin{aligned}
& s(x, y, t) \sim \sum_{j=0}^{\infty} s^{j}(x, y) t^{n-1-j} \quad \text { in } S_{1,0}^{n-1}\left(D \times D \times \mathbb{R}_{+}, T_{y}^{* 0, q} X \otimes T_{x}^{* 0, q} X\right), \\
& s^{j}(x, y) \in \mathscr{C}^{\infty}\left(D \times D, T_{y}^{* 0, q} X \otimes T_{x}^{* 0, q} X\right), \quad j \in \mathbb{N}_{0} .
\end{aligned}
$$

Definition 2.4. Let $Q \in L_{\mathrm{cl}}^{0}\left(X, T^{* 0, q} X \otimes T^{* 0, q} X\right)$ be a classical pseudodifferential operator on $X$. Let $D \Subset X$ be an open local coordinate patch of $X$ with local coordinates $x=$ $\left(x_{1}, \ldots, x_{2 n-1}\right)$ and let $\eta=\left(\eta_{1}, \ldots, \eta_{2 n-1}\right)$ be the dual variables of $x$. We write

$$
Q \equiv 0 \text { at } \Sigma^{-} \cap T^{*} D,
$$

if for every $D^{\prime} \Subset D$,

$$
Q(x, y) \equiv \int e^{i\langle x-y, \eta\rangle} q(x, \eta) d \eta \text { on } D^{\prime}
$$

where $q(x, \eta) \in S_{\mathrm{cl}}^{0}\left(T^{*} D^{\prime}, T^{* 0, q} X \otimes T^{* 0, q} X\right)$ and there exist $M>0$ and a conic open neighbourhood $\Lambda_{-}$of $\Sigma^{-}$such that for every $(x, \eta) \in T^{*} D^{\prime} \cap \Lambda_{-}$with $|\eta| \geq M$, we have $q(x, \eta)=0$. We define similarly $Q \equiv 0$ at $\Sigma^{+} \cap T^{*} D$. 


\section{Microlocal Hodge Decomposition Theorems For $\square_{b}^{(q)}$}

In this section we review some results in [44] about the existence of a microlocal Hodge decomposition of the Kohn Laplacian on an open set of a CR manifold where the Levi form is non-degenerate.

Theorems 3.1, Theorem 3.2 and Theorem 3.4 are proved in chapter 6 , chapter 7 and chapter 8 of part I in [44]. In [44] the existence of the microlocal Hodge decomposition is stated for compact CR manifolds, but the construction and arguments used are essentially local.

Theorem 3.1. We assume that the Levi form is non-degenerate of constant signature $\left(n_{-}, n_{+}\right)$at each point of an open set $D \Subset X$. Let $q \neq n_{-}, n_{+}$. Then, there is a properly supported operator $A \in L_{\frac{1}{2}, \frac{1}{2}}^{-1}\left(D, T^{* 0, q} D \otimes T^{* 0, q} D\right)$ such that $\square_{b}^{(q)} A \equiv I$ on $D$.

Let $p_{0}(x, \xi) \in \mathscr{C}^{\infty}\left(T^{*} X\right)$ be the principal symbol of $\square_{b}^{(q)}$. Note that $p_{0}(x, \xi)$ is a polynomial of degree 2 in $\xi$. Recall that the characteristic manifold of $\square_{b}^{(q)}$ is given by $\Sigma=\Sigma^{+} \cup \Sigma^{-}$, where $\Sigma^{+}$and $\Sigma^{-}$are given by (2.13).

Theorem 3.2. We assume that the Levi form is non-degenerate of constant signature $\left(n_{-}, n_{+}\right)$ at each point of an open set $D \Subset X$. Let $q=n_{-}$or $n_{+}$. Then there exist properly supported continuous operators $A \in L_{\frac{1}{2}, \frac{1}{2}}^{-1}\left(D, T^{* 0, q} D \otimes T^{* 0, q} D\right), S_{-}, S_{+} \in L_{\frac{1}{2}, \frac{1}{2}}^{0}\left(D, T^{* 0, q} D \otimes T^{* 0, q} D\right)$, such that

$$
\begin{aligned}
& \square_{b}^{(q)} A+S_{-}+S_{+}=I \text { on } D, \\
& \square_{b}^{(q)} S_{-} \equiv 0 \text { on } D, \square_{b}^{(q)} S_{+} \equiv 0 \text { on } D, \\
& A \equiv A^{*} \text { on } D, S_{-} A \equiv 0 \text { on } D, S_{+} A \equiv 0 \text { on } D, \\
& S_{-} \equiv S_{-}^{*} \equiv S_{-}^{2} \text { on } D, \\
& S_{+} \equiv S_{+}^{*} \equiv S_{+}^{2} \text { on } D, \\
& S_{-} S_{+} \equiv S_{+} S_{-} \equiv 0 \text { on } D,
\end{aligned}
$$

where $A^{*}, S_{-}^{*}$ and $S_{+}^{*}$ are the formal adjoints of $A, S_{-}$and $S_{+}$with respect to $(\cdot \mid \cdot)$ respectively and $S_{-}(x, y)$ satisfies

$$
S_{-}(x, y) \equiv \int_{0}^{\infty} e^{i \varphi_{-}(x, y) t} s_{-}(x, y, t) d t \text { on } D
$$

with a symbol $s_{-}(x, y, t) \in S_{\mathrm{cl}}^{n-1}\left(D \times D \times \mathbb{R}_{+}, T_{y}^{* 0, q} X \otimes T_{x}^{* 0, q} X\right)$ as in (2.15), (2.16) and phase function $\varphi_{-}$such that $\varphi=\varphi_{-}$satisfies

$$
\begin{aligned}
& \varphi \in \mathscr{C}^{\infty}(D \times D), \quad \operatorname{Im} \varphi(x, y) \geq 0, \\
& \varphi(x, x)=0, \quad \varphi(x, y) \neq 0 \text { if } x \neq y, \\
& \left.d_{x} \varphi(x, y)\right|_{x=y}=-\omega_{0}(x),\left.\quad d_{y} \varphi(x, y)\right|_{x=y}=\omega_{0}(x), \\
& \varphi(x, y)=-\bar{\varphi}(y, x) .
\end{aligned}
$$

Moreover, there is a function $f \in \mathscr{C}^{\infty}(D \times D)$ such that

$$
p_{0}\left(x, \varphi_{x}^{\prime}(x, y)\right)-f(x, y) \varphi(x, y)
$$

vanishes to infinite order at $x=y$. Similarly,

$$
S_{+}(x, y) \equiv \int_{0}^{\infty} e^{i \varphi_{+}(x, y) t} s_{+}(x, y, t) d t \text { on } D
$$


with $s_{+}(x, y, t) \in S_{\mathrm{cl}}^{n-1}\left(D \times D \times \mathbb{R}_{+}, T_{y}^{* 0, q} X \otimes T_{x}^{* 0, q} X\right)$ as in (2.15), (2.16) and - $\bar{\varphi}_{+}(x, y)$ satisfies (3.2) and (3.3). Moreover, if $q \neq n_{+}$, then $s_{+}(x, y, t)$ vanishes to infinite order at $x=y$. If $q \neq n_{-}$, then $s_{-}(x, y, t)$ vanishes to infinite order at $x=y$.

The operators $S_{+}, S_{-}$are called approximate Szegó kernels.

Remark 3.3. With the notations and assumptions used in Theorem 3.2 , assume that $q=n_{-} \neq$ $n_{+}$. Since $s_{+}(x, y, t)$ vanishes to infinite order at $x=y$, we have $S_{+} \equiv 0$ on $D$. Similarly, if $q=n_{+} \neq n_{-}$. then $S_{-} \equiv 0$ on $D$.

The following result describes the phase function in local coordinates.

Theorem 3.4. We assume that the Levi form is non-degenerate of constant signature $\left(n_{-}, n_{+}\right)$at each point of an open set $D \Subset X$. For a given point $x_{0} \in D$, let $\left\{W_{j}\right\}_{j=1}^{n-1}$ be an orthonormal frame of $T^{1,0} X$ in a neighbourhood of $x_{0}$ such that the Levi form is diagonal at $x_{0}$, i.e. $\mathcal{L}_{x_{0}}\left(W_{j}, \bar{W}_{s}\right)=$ $\delta_{j, s} \mu_{j}, j, s=1, \ldots, n-1$. We take local coordinates $x=\left(x_{1}, \ldots, x_{2 n-1}\right), z_{j}=x_{2 j-1}+i x_{2 j}$, $j=1, \ldots, n-1$, defined on some neighbourhood of $x_{0}$ such that $\omega_{0}\left(x_{0}\right)=d x_{2 n-1}, x\left(x_{0}\right)=0$, and for some $c_{j} \in \mathbb{C}, j=1, \ldots, n-1$,

$$
W_{j}=\frac{\partial}{\partial z_{j}}-i \mu_{j} \bar{z}_{j} \frac{\partial}{\partial x_{2 n-1}}-c_{j} x_{2 n-1} \frac{\partial}{\partial x_{2 n-1}}+O\left(|x|^{2}\right), j=1, \ldots, n-1 .
$$

Set $y=\left(y_{1}, \ldots, y_{2 n-1}\right), w_{j}=y_{2 j-1}+i y_{2 j}, j=1, \ldots, n-1$. Then, for $\varphi_{-}$in Theorem 3.2, we have

$$
\operatorname{Im} \varphi_{-}(x, y) \geq c \sum_{j=1}^{2 n-2}\left|x_{j}-y_{j}\right|^{2}, \quad c>0,
$$

in some neighbourhood of $(0,0)$ and

$$
\begin{aligned}
& \varphi_{-}(x, y)=-x_{2 n-1}+y_{2 n-1}+i \sum_{j=1}^{n-1}\left|\mu_{j}\right|\left|z_{j}-w_{j}\right|^{2} \\
& \quad+\sum_{j=1}^{n-1}\left(i \mu_{j}\left(\bar{z}_{j} w_{j}-z_{j} \bar{w}_{j}\right)+c_{j}\left(-z_{j} x_{2 n-1}+w_{j} y_{2 n-1}\right)\right. \\
& \left.\quad+\bar{c}_{j}\left(-\bar{z}_{j} x_{2 n-1}+\bar{w}_{j} y_{2 n-1}\right)\right)+\left(x_{2 n-1}-y_{2 n-1}\right) f(x, y)+O\left(|(x, y)|^{3}\right),
\end{aligned}
$$

where $f$ is smooth and satisfies $f(0,0)=0, f(x, y)=\bar{f}(y, x)$.

The following formula for the leading term $s_{-}^{0}$ on the diagonal follows from [44, $\S 8$ ], its calculation being local in nature. For a given point $x_{0} \in D$, let $\left\{W_{j}\right\}_{j=1}^{n-1}$ be an orthonormal frame of $\left(T^{1,0} X,\langle\cdot \mid \cdot\rangle\right)$ near $x_{0}$, for which the Levi form is diagonal at $x_{0}$. Put

$$
\mathcal{L}_{x_{0}}\left(W_{j}, \bar{W}_{\ell}\right)=\mu_{j}\left(x_{0}\right) \delta_{j \ell}, \quad j, \ell=1, \ldots, n-1 .
$$

We will denote by

$$
\operatorname{det} \mathcal{L}_{x_{0}}=\prod_{j=1}^{n-1} \mu_{j}\left(x_{0}\right)
$$


Let $\left\{T_{j}\right\}_{j=1}^{n-1}$ denote the basis of $T^{* 0,1} X$, dual to $\left\{\bar{W}_{j}\right\}_{j=1}^{n-1}$. We assume that $\mu_{j}\left(x_{0}\right)<0$ if $1 \leq j \leq n_{-}$and $\mu_{j}\left(x_{0}\right)>0$ if $n_{-}+1 \leq j \leq n-1$. Put

$$
\begin{aligned}
& \mathcal{N}\left(x_{0}, n_{-}\right):=\left\{c T_{1}\left(x_{0}\right) \wedge \ldots \wedge T_{n_{-}}\left(x_{0}\right) ; c \in \mathbb{C}\right\}, \\
& \mathcal{N}\left(x_{0}, n_{+}\right):=\left\{c T_{n_{-}+1}\left(x_{0}\right) \wedge \ldots \wedge T_{n-1}\left(x_{0}\right) ; c \in \mathbb{C}\right\}
\end{aligned}
$$

and let

$$
\tau_{x_{0}, n_{-}}: T_{x_{0}}^{* 0, q} X \rightarrow \mathcal{N}\left(x_{0}, n_{-}\right), \quad \tau_{x_{0}, n_{+}}: T_{x_{0}}^{* 0, q} X \rightarrow \mathcal{N}\left(x_{0}, n_{+}\right),
$$

be the orthogonal projections onto $\mathcal{N}\left(x_{0}, n_{-}\right)$and $\mathcal{N}\left(x_{0}, n_{+}\right)$with respect to $\langle\cdot \mid \cdot\rangle$ respectively. We recall that $m(x)$ is the given smooth $2 n-1$ form on $X$ and $v(x)$ is the volume form induced by $\langle\cdot \mid \cdot\rangle$, see the discussion after (2.4).

Theorem 3.5. We assume that the Levi form is non-degenerate of constant signature $\left(n_{-}, n_{+}\right)$at each point of an open set $D \Subset X$. If $q=n_{-}$, then for the leading term $s_{-}^{0}(x, y)$ of the expansion (2.16) of $s_{-}(x, y, t)$, we have

$$
s_{-}^{0}\left(x_{0}, x_{0}\right)=\frac{1}{2} \pi^{-n}\left|\operatorname{det} \mathcal{L}_{x_{0}}\right| \frac{v\left(x_{0}\right)}{m\left(x_{0}\right)} \tau_{x_{0}, n_{-}}, x_{0} \in D .
$$

Similarly, if $q=n_{+}$, then for the leading term $s_{+}^{0}(x, y)$ of the expansion (2.16) of $s_{+}(x, y, t)$, we have

$$
s_{+}^{0}\left(x_{0}, x_{0}\right)=\frac{1}{2} \pi^{-n}\left|\operatorname{det} \mathcal{L}_{x_{0}}\right| \frac{v\left(x_{0}\right)}{m\left(x_{0}\right)} \tau_{x_{0}, n_{+}}, x_{0} \in D
$$

\section{Microlocal SPECTRAL THEORY FOR $\square_{b}^{(q)}$}

In this section, we will apply the microlocal Hodge decomposition theorems for $\square_{b}^{(q)}$ from Section 3 in order to study the singularities for the kernel $\Pi_{\leq \lambda}^{(q)}(x, y)$ on the non-degenerate part of the Levi form. The section ends with the proof of Theorem 1.7.

For any $\lambda>0$, it is clearly that there is a continuous operator

$$
N_{\lambda}^{(q)}: L_{(0, q)}^{2}(X) \rightarrow \operatorname{Dom} \square_{b}^{(q)}
$$

such that

$$
\begin{aligned}
& \square_{b}^{(q)} N_{\lambda}^{(q)}+\Pi_{\leq \lambda}^{(q)}=I \text { on } L_{(0, q)}^{2}(X), \\
& N_{\lambda}^{(q)} \square_{b}^{(q)}+\Pi_{\leq \lambda}^{(q)}=I \text { on } \operatorname{Dom} \square_{b}^{(q)} .
\end{aligned}
$$

Let us formulate a detailed version of of Theorem 1.5 .

Theorem 4.1. With the notations and assumptions used above, assume that the Levi form is nondegenerate of constant signature $\left(n_{-}, n_{+}\right)$at each point of an open set $D \Subset X$. If $q \notin\left\{n_{-}, n_{+}\right\}$, then there is a $A \in L_{\frac{1}{2}, \frac{1}{2}}^{-1}\left(D, T^{* 0, q} X \otimes T^{* 0, q} X\right)$, such that for any $\lambda>0$, we have

$$
\Pi_{\leq \lambda}^{(q)} \equiv 0 \quad \text { and } \quad N_{\lambda}^{(q)} \equiv A \quad \text { on } D .
$$

If $q \in\left\{n_{-}, n_{+}\right\}$, then for any $\lambda>0$, we have

$$
\Pi_{\leq \lambda}^{(q)} \equiv S_{-}+S_{+} \quad \text { and } \quad N_{\lambda}^{(q)} \equiv G \quad \text { on } D,
$$


where $G \in L_{\frac{1}{2}, \frac{1}{2}}^{-1}\left(D, T^{* 0, q} X \otimes T^{* 0, q} X\right), S_{-}, S_{+} \in L_{\frac{1}{2}, \frac{1}{2}}^{0}\left(D, T^{* 0, q} X \otimes T^{* 0, q} X\right)$ are independent of $\lambda$ and the kernels of $S_{-}$and $S_{+}$satisfy

$$
S_{ \pm}(x, y) \equiv \int_{0}^{\infty} e^{i \varphi_{ \pm}(x, y) t} s_{ \pm}(x, y, t) d t \text { on } D
$$

with symbols $s_{ \pm}(x, y, t) \in S_{\mathrm{cl}}^{n-1}\left(D \times D \times \mathbb{R}_{+}, T_{y}^{* 0, q} X \otimes T_{x}^{* 0, q} X\right)$ as in (2.15), (2.16), $s_{-}=0$ if $q \neq n_{-}, s_{+}=0$ if $q \neq n_{+}$, where $s_{-}^{0}(x, x)$ and $s_{+}^{0}(x, x)$ are given by (4.2), and phase functions $\varphi_{ \pm}$such that $\varphi=\varphi_{-}$and $\varphi=-\bar{\varphi}_{+}$satisfy (3.2), (3.3) (see Theorem 4.2 and Theorem 4.4 for more properties of the phases $\varphi_{ \pm}$).

Since $s_{-}(x, y, t)=0$ if $q \neq n_{-}, S_{-} \equiv 0$ on $D$ if $q \neq n_{-}$. Similarly, $S_{+} \equiv 0$ on $D$ if $q \neq n_{+}$. The following result describes the phase function in local coordinates.

Theorem 4.2. The function $\varphi_{-}$from Theorem 4.1 fulfills the estimates (3.4) and (3.5) in local coordinates near a point of $D$, chosen as in Theorem 3.4

Definition 4.3. With the assumptions and notations used in Theorem 4.1 , let $\varphi_{1}, \varphi_{2} \in \mathscr{C}^{\infty}(D \times$ $D)$. We assume that $\varphi_{1}$ and $\varphi_{2}$ satisfy (3.2) and (3.4). We say that $\varphi_{1}$ and $\varphi_{2}$ are equivalent on $D$ if for any $b_{1}(x, y, t) \in S_{\mathrm{cl}}^{n-1}\left(D \times D \times \mathbb{R}_{+}, T_{y}^{* 0, q} X \otimes T_{x}^{* 0, q} X\right)$ we can find $b_{2}(x, y, t) \in$ $S_{\mathrm{cl}}^{n-1}\left(D \times D \times \mathbb{R}_{+}, T_{y}^{* 0, q} X \otimes T_{x}^{* 0, q} X\right)$ such that

$$
\int_{0}^{\infty} e^{i \varphi_{1}(x, y) t} b_{1}(x, y, t) d t \equiv e^{i \varphi_{2}(x, y) t} b_{2}(x, y, t) d t \text { on } D
$$

and vise versa.

We characterize now the phase $\varphi_{-}$(see Section 8).

Theorem 4.4. With the assumptions and notations used in Theorem 4.1 let $\varphi_{1} \in \mathscr{C}^{\infty}(D \times D)$. We assume that $\varphi_{1}$ satisfies (3.2) and (3.4). The functions $\varphi_{1}$ and $\varphi_{-}$are equivalent on $D$ in the sense of Definition 4.3 if and only if there is a function $h \in \mathscr{C}^{\infty}(D \times D)$ such that $\varphi_{1}(x, y)-h(x, y) \varphi_{-}(x, y)$ vanishes to infinite order at $x=y$.

The proof of Theorem 4.4 is essentially the same as the proof of Theorem 5.4 and therefore will be omitted.

We give the formulas of the leading terms of the asymptotic expansions of the symbols $s_{ \pm}(x, y)$ from Theorem 4.1.

Theorem 4.5. With the assumptions and notations used in Theorem 4.1 and the notations (3.6), (3.8), (3.9), we have for a given point $x_{0} \in D$,

$$
\begin{aligned}
& s_{-}^{0}\left(x_{0}, x_{0}\right)=\frac{1}{2} \pi^{-n}\left|\operatorname{det} \mathcal{L}_{x_{0}}\right| \frac{v\left(x_{0}\right)}{m\left(x_{0}\right)} \tau_{x_{0}, n_{-}}, \text {for } q=n_{-}, \\
& s_{+}^{0}\left(x_{0}, x_{0}\right)=\frac{1}{2} \pi^{-n}\left|\operatorname{det} \mathcal{L}_{x_{0}}\right| \frac{v\left(x_{0}\right)}{m\left(x_{0}\right)} \tau_{x_{0}, n_{+}}, \text {for } q=n_{+} .
\end{aligned}
$$

Recall that $\Pi_{\leq \lambda}^{(q)}$ is given by (2.11). Let $\lambda \geq 0$. From the spectral theory for self-adjoint operators (see Davies [25]), it is well-known that

$$
\Pi_{\leq \lambda}^{(q)}: L_{(0, q)}^{2}(X) \rightarrow \operatorname{Dom} \square_{b}^{(q)}, \quad \Pi_{\leq \lambda}^{(q)} \square_{b}^{(q)}=\square_{b}^{(q)} \Pi_{\leq \lambda}^{(q)} \text { on } \operatorname{Dom} \square_{b}^{(q)}
$$


and $\Pi_{\leq \lambda}^{(q)} \square_{b}^{(q)}: \operatorname{Dom} \square_{b}^{(q)} \rightarrow L_{(0, q)}^{2}(X)$ is continuous. Since Dom $\square_{b}^{(q)}$ is dense in $L_{(0, q)}^{2}(X)$, we can extend $\Pi_{\leq \lambda}^{(q)} \square_{b}^{(q)}$ continuously to $L_{(0, q)}^{2}(X)$ in the standard way. Similarly, for every $m \in \mathbb{N}$, we can extend $\Pi_{\leq \lambda}^{(q)}\left(\square_{b}^{(q)}\right)^{m}$ continuously to $L_{(0, q)}^{2}(X)$ and we have

$$
\begin{aligned}
& \left(\square_{b}^{(q)}\right)^{m} \Pi_{\leq \lambda}^{(q)}=\Pi_{\leq \lambda}^{(q)}\left(\square_{b}^{(q)}\right)^{m} \text { on } L_{(0, q)}^{2}(X), \\
& \left(\square_{b}^{(q)}\right)^{m} \Pi_{\leq \lambda}^{(q)}=\Pi_{\leq \lambda}^{(q)}\left(\square_{b}^{(q)}\right)^{m}: L_{(0, q)}^{2}(X) \rightarrow \operatorname{Dom} \square_{b}^{(q)} \text { is continuous. }
\end{aligned}
$$

Now, we fix $\lambda>0$. It is clearly that there is a continuous operator

$$
N_{\lambda}^{(q)}: L_{(0, q)}^{2}(X) \rightarrow \operatorname{Dom} \square_{b}^{(q)}
$$

such that

$$
\begin{aligned}
& \square_{b}^{(q)} N_{\lambda}^{(q)}+\Pi_{\leq \lambda}^{(q)}=I \text { on } L_{(0, q)}^{2}(X), \\
& N_{\lambda}^{(q)} \square_{b}^{(q)}+\Pi_{\leq \lambda}^{(q)}=I \text { on } \operatorname{Dom} \square_{b}^{(q)} .
\end{aligned}
$$

Until further notice, we assume that the Levi form is non-degenerate of constant signature $\left(n_{-}, n_{+}\right)$at each point of an open set $D \Subset X$ and we work on $D$. We need

Theorem 4.6. With the assumptions and notations used above, let $q=n_{-}$or $n_{+}$. We have

$$
\square_{b}^{(q)} \Pi_{\leq \lambda}^{(q)} \equiv 0 \text { on } D
$$

Proof. In view of (3.1), we see that

$$
A^{*} \square_{b}^{(q)}+S_{-}^{*}+S_{+}^{*}=I \text { on } D .
$$

Note that $A^{*}, S_{-}^{*}, S_{+}^{*}, A, S_{-}$and $S_{+}$are properly supported. We recall that

$$
\begin{aligned}
& A^{*}, A: H_{\mathrm{comp}}^{s}\left(D, T^{* 0, q} X\right) \rightarrow H_{\mathrm{comp}}^{s+1}\left(D, T^{* 0, q} X\right), \quad \forall s \in \mathbb{Z}, \\
& A^{*}, A: H_{\mathrm{loc}}^{s}\left(D, T^{* 0, q} X\right) \rightarrow H_{\mathrm{loc}}^{s+1}\left(D, T^{* 0, q} X\right), \quad \forall s \in \mathbb{Z}, \\
& S_{-}^{*}, S_{-}, S_{+}^{*}, S_{+}: H_{\mathrm{comp}}^{s}\left(D, T^{* 0, q} X\right) \rightarrow H_{\mathrm{comp}}^{s}\left(D, T^{* 0, q} X\right), \quad \forall s \in \mathbb{Z}, \\
& S_{-}^{*}, S_{-}, S_{+}^{*}, S_{+}: H_{\mathrm{loc}}^{s}\left(D, T^{* 0, q} X\right) \rightarrow H_{\mathrm{loc}}^{s}\left(D, T^{* 0, q} X\right), \quad \forall s \in \mathbb{Z} .
\end{aligned}
$$

From (4.5), we have

$$
A^{*}\left(\square_{b}^{(q)}\right)^{2} \Pi_{\leq \lambda}^{(q)}+\left(S_{-}^{*}+S_{+}^{*}\right) \square_{b}^{(q)} \Pi_{\leq \lambda}^{(q)}=\square_{b}^{(q)} \Pi_{\leq \lambda}^{(q)} .
$$

Since $\left(S_{-}^{*}+S_{+}^{*}\right) \square_{b}^{(q)} \equiv 0$ on $D$, we have

$$
\left(S_{-}^{*}+S_{+}^{*}\right) \square_{b}^{(q)} \Pi_{\leq \lambda}^{(q)}: H_{\mathrm{comp}}^{0}\left(D, T^{* 0, q} X\right) \rightarrow H_{\mathrm{loc}}^{s}\left(D, T^{* 0, q} X\right), \quad \forall s \in \mathbb{N}_{0} .
$$

From (4.3) and (4.6), we see that

$$
A^{*}\left(\square_{b}^{(q)}\right)^{2} \Pi_{\leq \lambda}^{(q)}: H_{\text {comp }}^{0}\left(D, T^{* 0, q} X\right) \rightarrow H_{\mathrm{loc}}^{1}\left(D, T^{* 0, q} X\right) .
$$

From (4.9), (4.8) and (4.7), we conclude that

$$
\square_{b}^{(q)} \Pi_{\leq \lambda}^{(q)}: H_{\text {comp }}^{0}\left(D, T^{* 0, q} X\right) \rightarrow H_{\text {loc }}^{1}\left(D, T^{* 0, q} X\right) .
$$

Similarly, we can repeat the procedure above and deduce that

$$
\left(\square_{b}^{(q)}\right)^{2} \Pi_{\leq \lambda}^{(q)}: H_{\text {comp }}^{0}\left(D, T^{* 0, q} X\right) \rightarrow H_{\mathrm{loc}}^{1}\left(D, T^{* 0, q} X\right) .
$$

From (4.11) and (4.6), we get

$$
A^{*}\left(\square_{b}^{(q)}\right)^{2} \Pi_{\leq \lambda}^{(q)}: H_{\mathrm{comp}}^{0}\left(D, T^{* 0, q} X\right) \rightarrow H_{\mathrm{loc}}^{2}\left(D, T^{* 0, q} X\right) .
$$


Combining (4.12), (4.8) with (4.7), we obtain

$$
\square_{b}^{(q)} \Pi_{\leq \lambda}^{(q)}: H_{\text {comp }}^{0}\left(D, T^{* 0, q} X\right) \rightarrow H_{\mathrm{loc}}^{2}\left(D, T^{* 0, q} X\right) .
$$

Continuing in this way, we deduce that

$$
\square_{b}^{(q)} \Pi_{\leq \lambda}^{(q)}: H_{\mathrm{comp}}^{0}\left(D, T^{* 0, q} X\right) \rightarrow H_{\mathrm{loc}}^{s}\left(D, T^{* 0, q} X\right), \quad \forall s \in \mathbb{N}_{0} .
$$

Since $\square_{b}^{(q)} \Pi_{\leq \lambda}^{(q)}=\Pi_{\leq \lambda}^{(q)} \square_{b}^{(q)}$,

$$
\Pi_{\leq \lambda}^{(q)} \square_{b}^{(q)}: H_{\text {comp }}^{0}\left(D, T^{* 0, q} X\right) \rightarrow H_{\text {loc }}^{s}\left(D, T^{* 0, q} X\right), \quad \forall s \in \mathbb{N}_{0} .
$$

By taking adjoint in (4.15), we conclude that

$$
\square_{b}^{(q)} \Pi_{\leq \lambda}^{(q)}: H_{\text {comp }}^{-s}\left(D, T^{* 0, q} X\right) \rightarrow H_{\mathrm{loc}}^{0}\left(D, T^{* 0, q} X\right), \quad \forall s \in \mathbb{N}_{0} .
$$

Similarly, we can repeat the procedure above and deduce that for every $m \in \mathbb{N}$,

$$
\begin{aligned}
& \left(\square_{b}^{(q)}\right)^{m} \Pi_{\leq \lambda}^{(q)}: H_{\text {comp }}^{-s}\left(D, T^{* 0, q} X\right) \rightarrow H_{\mathrm{loc}}^{0}\left(D, T^{* 0, q} X\right), \quad \forall s \in \mathbb{N}_{0}, \\
& \left(\square_{b}^{(q)}\right)^{m} \Pi_{\leq \lambda}^{(q)}: H_{\text {comp }}^{0}\left(D, T^{* 0, q} X\right) \rightarrow H_{\mathrm{loc}}^{s}\left(D, T^{* 0, q} X\right), \quad \forall s \in \mathbb{N}_{0} .
\end{aligned}
$$

Now, from (4.4), we have

$$
\left(S_{-}^{*}+S_{+}^{*}\right) \square_{b}^{(q)} N_{\lambda}^{(q)}+\left(S_{-}^{*}+S_{+}^{*}\right) \Pi_{\leq \lambda}^{(q)}=S_{-}^{*}+S_{+}^{*} .
$$

Since $\left(S_{-}^{*}+S_{+}^{*}\right) \square_{b}^{(q)} \equiv 0$ on $D$, from (4.18), it is easy to see that

$$
\left(S_{-}^{*}+S_{+}^{*}\right)-\left(S_{-}^{*}+S_{+}^{*}\right) \Pi_{\leq \lambda}^{(q)}: H_{\text {comp }}^{0}\left(D, T^{* 0, q} X\right) \rightarrow H_{\text {loc }}^{s}\left(D, T^{* 0, q} X\right), \quad \forall s \in \mathbb{N}_{0} .
$$

From (4.5), we have

$$
A^{*} \square_{b}^{(q)} \Pi_{\leq \lambda}^{(q)}+\left(S_{-}^{*}+S_{+}^{*}\right) \Pi_{\leq \lambda}^{(q)}=\Pi_{\leq \lambda}^{(q)} .
$$

From (4.6), (4.17), (4.20) and (4.19), it is not difficult to see that

$$
\left(S_{-}^{*}+S_{+}^{*}\right)-\Pi_{\leq \lambda}^{(q)}: H_{\text {comp }}^{0}\left(D, T^{* 0, q} X\right) \rightarrow H_{\text {loc }}^{s}\left(D, T^{* 0, q} X\right), \quad \forall s \in \mathbb{N}_{0}
$$

and hence

$$
\left(S_{-}+S_{+}\right)-\Pi_{\leq \lambda}^{(q)}: H_{\mathrm{comp}}^{-s}\left(D, T^{* 0, q} X\right) \rightarrow H_{\mathrm{loc}}^{0}\left(D, T^{* 0, q} X\right), \quad \forall s \in \mathbb{N}_{0} .
$$

Combining (4.22) with (4.6), we deduce that for any $s \in \mathbb{N}_{0}$ we can extend $\Pi_{\leq \lambda}^{(q)}$ to the space $H_{\text {comp }}^{-s}\left(D, T^{* 0, q} X\right)$, and we have

$$
\Pi_{\leq \lambda}^{(q)}: H_{\text {comp }}^{-s}\left(D, T^{* 0, q} X\right) \rightarrow H_{\mathrm{loc}}^{-s}\left(D, T^{* 0, q} X\right), \quad \forall s \in \mathbb{N}_{0} .
$$

From (4.23) and note that $\left(S_{-}^{*}+S_{+}^{*}\right) \square_{b}^{(q)} \equiv 0$ on $D$, we have

$$
\left(S_{-}^{*}+S_{+}^{*}\right) \square_{b}^{(q)} \Pi_{\leq \lambda}^{(q)}: H_{\text {comp }}^{-s}\left(D, T^{* 0, q} X\right) \rightarrow H_{\text {loc }}^{s}\left(D, T^{* 0, q} X\right), \quad \forall s \in \mathbb{N}_{0} .
$$

From (4.24), (4.17), (4.7) and (4.6), we obtain

$$
\square_{b}^{(q)} \Pi_{\leq \lambda}^{(q)}: H_{\text {comp }}^{-s}\left(D, T^{* 0, q} X\right) \rightarrow H_{\text {loc }}^{1}\left(D, T^{* 0, q} X\right), \quad \forall s \in \mathbb{N}_{0} .
$$

Similarly, we can repeat the procedure above and deduce that

$$
\left(\square_{b}^{(q)}\right)^{2} \Pi_{\leq \lambda}^{(q)}: H_{\text {comp }}^{-s}\left(D, T^{* 0, q} X\right) \rightarrow H_{\mathrm{loc}}^{1}\left(D, T^{* 0, q} X\right), \quad \forall s \in \mathbb{N}_{0} .
$$

From (4.26) and (4.6), we get

$$
A^{*}\left(\square_{b}^{(q)}\right)^{2} \Pi_{\leq \lambda}^{(q)}: H_{\text {comp }}^{-s}\left(D, T^{* 0, q} X\right) \rightarrow H_{\text {loc }}^{2}\left(D, T^{* 0, q} X\right), \quad \forall s \in \mathbb{N}_{0} .
$$


Combining (4.27), (4.24) with (4.7), we obtain

$$
\square_{b}^{(q)} \Pi_{\leq \lambda}^{(q)}: H_{\text {comp }}^{-s}\left(D, T^{* 0, q} X\right) \rightarrow H_{\mathrm{loc}}^{2}\left(D, T^{* 0, q} X\right), \quad \forall s \in \mathbb{N}_{0} .
$$

Continuing in this way, we deduce that

$$
\square_{b}^{(q)} \Pi_{\leq \lambda}^{(q)}: H_{\text {comp }}^{-s}\left(D, T^{* 0, q} X\right) \rightarrow H_{\mathrm{loc}}^{\ell}\left(D, T^{* 0, q} X\right), \quad \forall s, \ell \in \mathbb{N}_{0} .
$$

Hence, $\square_{b}^{(q)} \Pi_{\leq \lambda}^{(q)} \equiv 0$ on $D$. The theorem follows.

Now, we can prove one of the main results of this work.

Theorem 4.7. We assume that the Levi form is non-degenerate of constant signature $\left(n_{-}, n_{+}\right)$ at each point of an open set $D \Subset X$. Let $q=n_{-}$or $n_{+}$. Then, for any $\lambda>0$, we have

$$
\Pi_{\leq \lambda}^{(q)} \equiv S_{-}+S_{+} \quad \text { and } \quad N_{\lambda}^{(q)} \equiv A \quad \text { on } D,
$$

where $N_{\lambda}^{(q)}$ is given by (4.4), $S_{-}, S_{+}$and $A$ are as in Theorem 3.2

Proof. Fix $\lambda>0$. From (4.5), we have

$$
A^{*} \square_{b}^{(q)} \Pi_{\leq \lambda}^{(q)}+\left(S_{-}^{*}+S_{+}^{*}\right) \Pi_{\leq \lambda}^{(q)}=\Pi_{\leq \lambda}^{(q)} \text { on } D .
$$

In view of Theorem 4.6, we see that

$$
\left(S_{-}^{*}+S_{+}^{*}\right) \Pi_{\leq \lambda}^{(q)}=\Pi_{\leq \lambda}^{(q)}-F_{1} \text { on } D,
$$

where

$$
\begin{aligned}
& F_{1}=A^{*} \square_{b}^{(q)} \Pi_{\leq \lambda}^{(q)}, \\
& F_{1} \equiv 0 \text { on } D .
\end{aligned}
$$

On the other hand, from (4.4), we have

$$
N_{\lambda}^{(q)} \square_{b}^{(q)}\left(S_{-}+S_{+}\right)+\Pi_{\leq \lambda}^{(q)}\left(S_{-}+S_{+}\right)=S_{-}+S_{+} \cdot
$$

Since $\square_{b}^{(q)}\left(S_{-}+S_{+}\right) \equiv 0$ on $D$, we conclude that

$$
\begin{aligned}
& S_{-}+S_{+}=\Pi_{\leq \lambda}^{(q)}\left(S_{-}+S_{+}\right)+N_{\lambda}^{(q)} F, \\
& S_{-}^{*}+S_{+}^{*}=\left(S_{-}^{*}+S_{+}^{*}\right) \Pi_{\leq \lambda}^{(q)}+F^{*} N_{\lambda}^{(q)},
\end{aligned}
$$

where $F \equiv 0$ on $D$ and $F^{*}$ is the adjoint of $F$. Note that $F$ and $F^{*}$ are properly supported on $D$. From (4.29) and (4.31), we deduce that

$$
\begin{aligned}
& S_{-}+S_{+}+F_{1}^{*}=\Pi_{\leq \lambda}^{(q)}+N_{\lambda}^{(q)} F, \\
& S_{-}^{*}+S_{+}^{*}+F_{1}=\Pi_{\leq \lambda}^{(q)}+F^{*} N_{\lambda}^{(q)},
\end{aligned}
$$

where $F_{1}^{*}$ is the adjoint of $F_{1}$. From (4.32), we have

$$
\left(S_{-}^{*}+S_{+}^{*}+F_{1}-\Pi_{\leq \lambda}^{(q)}\right)\left(S_{-}+S_{+}+F_{1}^{*}-\Pi_{\leq \lambda}^{(q)}\right)=F^{*}\left(N_{\lambda}^{(q)}\right)^{2} F \text { on } H_{\mathrm{comp}}^{0}\left(D, T^{* 0, q} X\right) .
$$

Since

$$
F^{*}\left(N_{\lambda}^{(q)}\right)^{2} F: \mathscr{E}^{\prime}\left(D, T^{* 0, q} X\right) \rightarrow \Omega_{0}^{0, q}(X) \subset L_{(0, q)}^{2}(X) \rightarrow \Omega^{0, q}(X),
$$

we have $F^{*}\left(N_{\lambda}^{(q)}\right)^{2} F \equiv 0$ on $D$. From this observation and (4.33), we obtain

$$
\left(S_{-}^{*}+S_{+}^{*}+F_{1}-\Pi_{\leq \lambda}^{(q)}\right)\left(S_{-}+S_{+}+F_{1}^{*}-\Pi_{\leq \lambda}^{(q)}\right) \equiv 0 \text { on } D .
$$


Now,

$$
\begin{aligned}
& \left(S_{-}^{*}+S_{+}^{*}+F_{1}-\Pi_{\leq \lambda}^{(q)}\right)\left(S_{-}+S_{+}+F_{1}^{*}-\Pi_{\leq \lambda}^{(q)}\right) \\
& =\left(S_{-}^{*}+S_{+}^{*}\right)\left(S_{-}+S_{+}\right)+\left(S_{-}^{*}+S_{+}^{*}\right) F_{1}^{*}-\left(S_{-}^{*}+S_{+}^{*}\right) \Pi_{\leq \lambda}^{(q)}+F_{1}\left(S_{-}+S_{+}\right) \\
& \quad+F_{1} F_{1}^{*}-F_{1} \Pi_{\leq \lambda}^{(q)}-\Pi_{\leq \lambda}^{(q)}\left(S_{-}+S_{+}\right)-\Pi_{\leq \lambda}^{(q)} F_{1}^{*}+\Pi_{\leq \lambda}^{(q)} .
\end{aligned}
$$

Since $F_{1} \equiv 0$ on $D$ and $S_{-}, S_{+}$are properly supported on $D$, it is clearly that $F_{1}\left(S_{-}+S_{+}\right)$and $\left(S_{-}^{*}+S_{+}^{*}\right) F_{1}^{*}$ are well-defined and

$$
F_{1}\left(S_{-}+S_{+}\right) \equiv 0, \quad\left(S_{-}^{*}+S_{+}^{*}\right) F_{1}^{*} \equiv 0 \quad \text { on } D .
$$

From (4.30) and Theorem 4.6, we see that

$$
F_{1} \Pi_{\leq \lambda}^{(q)}=A^{*} \square_{b}^{(q)}\left(\Pi_{\leq \lambda}^{(q)}\right)^{2}=A^{*} \square_{b}^{(q)} \Pi_{\leq \lambda}^{(q)} \equiv 0 \text { on } D
$$

and hence

$$
\Pi_{\leq \lambda}^{(q)} F_{1}^{*} \equiv 0 \text { on } D
$$

From (4.30), we see that $F_{1} F_{1}^{*}=A^{*}\left(\square_{b}^{(q)}\right)^{2} \Pi_{\leq \lambda}^{(q)} A$. Since $A$ is properly supported, $F_{1} F_{1}^{*}$ is well-defined as a continuous operator

$$
F_{1} F_{1}^{*}: \Omega_{0}^{0, q}(D) \rightarrow \mathscr{D}^{\prime}\left(D, T^{* 0, q} X\right) .
$$

Moreover, from the proof of Theorem 4.6, we see that $\left(\square_{b}^{(q)}\right)^{2} \Pi_{\leq \lambda}^{(q)} \equiv 0$ on $D$. Thus,

$$
F_{1} F_{1}^{*}=A^{*}\left(\square_{b}^{(q)}\right)^{2} \Pi_{\leq \lambda}^{(q)} A \equiv 0 \text { on } D .
$$

From (4.29), (4.35), (4.36), (4.37), (4.38) and (4.39), it is straightforward to see that

$$
\begin{aligned}
& \left(S_{-}^{*}+S_{+}^{*}+F_{1}-\Pi_{\leq \lambda}^{(q)}\right)\left(S_{-}+S_{+}+F_{1}^{*}-\Pi_{\leq \lambda}^{(q)}\right) \\
& \quad \equiv\left(S_{-}^{*}+S_{+}^{*}\right)\left(S_{-}+S_{+}\right)-\Pi_{\leq \lambda}^{(q)} \text { on } D .
\end{aligned}
$$

From (4.40) and (4.34), we conclude that

$$
\left(S_{-}^{*}+S_{+}^{*}\right)\left(S_{-}+S_{+}\right) \equiv \Pi_{\leq \lambda}^{(q)} \text { on } D .
$$

From (3.1), it is not difficult to see that $\left(S_{-}^{*}+S_{+}^{*}\right)\left(S_{-}+S_{+}\right) \equiv S_{-}+S_{+}$on $D$. Combining this observation with (4.41), we get

$$
S_{-}+S_{+} \equiv \Pi_{\leq \lambda}^{(q)} \text { on } D .
$$

The first formula in (4.28) follows. We now prove the second formula in (4.28). We first claim that

$$
\left(S_{-}^{*}+S_{+}^{*}\right) N_{\lambda}^{(q)}\left(S_{-}+S_{+}\right) \equiv 0 \text { on } D .
$$

From (4.32), (4.30) and notice that $N_{\lambda}^{(q)} \Pi_{\leq \lambda}^{(q)}=\Pi_{\leq \lambda}^{(q)} N_{\lambda}^{(q)}=0$, we have

$$
N_{\lambda}^{(q)}\left(S_{-}+S_{+}\right)=\left(N_{\lambda}^{(q)}\right)^{2} F
$$

and hence

$$
\left(N_{\lambda}^{(q)}\right)^{2}\left(S_{-}+S_{+}\right)=\left(N_{\lambda}^{(q)}\right)^{3} F .
$$


From (4.32), (4.30) and (4.44), we have

$$
\begin{aligned}
\left(S_{-}^{*}+S_{+}^{*}\right) N_{\lambda}^{(q)}\left(S_{-}+S_{+}\right) & =\left(\Pi_{\leq \lambda}^{(q)}+F^{*} N_{\lambda}^{(q)}-F_{1}\right) N_{\lambda}^{(q)}\left(S_{-}+S_{+}\right) \\
& =F^{*}\left(N_{\lambda}^{(q)}\right)^{2}\left(S_{-}+S_{+}\right) \\
& =F^{*}\left(N_{\lambda}^{(q)}\right)^{3} F \equiv 0 \text { on } D .
\end{aligned}
$$

The claim (4.43) follows. On $D$, we have

$$
N_{\lambda}^{(q)}=\left(A^{*} \square_{b}^{(q)}+S_{-}^{*}+S_{+}^{*}\right) N_{\lambda}^{(q)}=A^{*}\left(I-\Pi_{\leq \lambda}^{(q)}\right)+\left(S_{-}^{*}+S_{+}^{*}\right) N_{\lambda}^{(q)}
$$

and

$$
N_{\lambda}^{(q)}=N_{\lambda}^{(q)}\left(\square_{b}^{(q)} A+S_{-}+S_{+}\right)=\left(I-\Pi_{\leq \lambda}^{(q)}\right) A+N_{\lambda}^{(q)}\left(S_{-}+S_{+}\right) .
$$

From (4.46) and (4.47), we have

$$
\begin{aligned}
N_{\lambda}^{(q)} & =\left(I-\Pi_{\leq \lambda}^{(q)}\right) A+N_{\lambda}^{(q)}\left(S_{-}+S_{+}\right) \\
& =A-\Pi_{\leq \lambda}^{(q)} A+\left(A^{*}\left(I-\Pi_{\leq \lambda}^{(q)}\right)+\left(S_{-}^{*}+S_{+}^{*}\right) N_{\lambda}^{(q)}\right)\left(S_{-}+S_{+}\right) \\
& =A-\Pi_{\leq \lambda}^{(q)} A+A^{*}\left(I-\Pi_{\leq \lambda}^{(q)}\right)\left(S_{-}+S_{+}\right)+\left(S_{-}^{*}+S_{+}^{*}\right) N_{\lambda}^{(q)}\left(S_{-}+S_{+}\right) .
\end{aligned}
$$

From (4.43), (4.42) and noting that $\left(S_{-}+S_{+}\right) A \equiv 0$ on $D, A^{*}\left(S_{-}+S_{+}\right) \equiv 0$ on $D$, we conclude that

$$
\Pi_{\leq \lambda}^{(q)} A+A^{*}\left(I-\Pi_{\leq \lambda}^{(q)}\right)\left(S_{-}+S_{+}\right)+\left(S_{-}^{*}+S_{+}^{*}\right) N_{\lambda}^{(q)}\left(S_{-}+S_{+}\right) \equiv 0 \text { on } D .
$$

From this and (4.48), we get the second formula in (4.28). The theorem follows.

By using Theorem 3.1, we can repeat the proof of Theorem 4.7 and conclude the following.

Theorem 4.8. We assume that the Levi form is non-degenerate of constant signature $\left(n_{-}, n_{+}\right)$ at each point of an open set $D \Subset X$. Assume that $q \notin\left\{n_{-}, n_{+}\right\}$. Then, for any $\lambda>0$, we have

$$
\Pi_{\leq \lambda}^{(q)} \equiv 0 \quad \text { and } \quad N_{\lambda}^{(q)} \equiv A \quad \text { on } D
$$

where $N_{\lambda}^{(q)}$ is given by (4.4), and $A$ is as in Theorem 3.1.

From Theorem 4.7 and Theorem 4.8 , Theorem 4.1 follows.

Definition 4.9. Let $H$ be a Hilbert space and $Q$ be a closed densely defined operator $Q$ : Dom $Q \subset H \rightarrow \operatorname{Ran} Q \subset H$, with closed range. By the partial inverse of $Q$, we mean the bounded operator $M: H \rightarrow H$ such that $Q \circ M=\pi_{2}, M \circ Q=\pi_{1}$ on $\operatorname{Dom} Q$, where $\pi_{1}, \pi_{2}$ are the orthogonal projections in $H$ such that $\operatorname{Ran} \pi_{1}=(\operatorname{Ker} Q)^{\perp}, \operatorname{Ran} \pi_{2}=\operatorname{Ran} Q$. In other words, for $u \in H$, let $\pi_{2} u=Q v, v \in(\operatorname{Ker} Q)^{\perp} \cap \operatorname{Dom} Q$. Then, $M u=v$.

From Theorem 4.7 and Theorem 4.8, we deduce:

Corollary 4.10. Let $q \in\{0,1, \ldots, n-1\}$. Assume that $\square_{b}^{(q)}$ has $L^{2}$ closed range and let $N^{(q)}$ : $L_{(0, q)}^{2}(X) \rightarrow \operatorname{Dom} \square_{b}^{(q)}$ be the partial inverse of $\square_{b}^{(q)}$. We assume that the Levi form is nondegenerate of constant signature $\left(n_{-}, n_{+}\right)$at each point of an open set $D \Subset X$. If $q \notin\left\{n_{-}, n_{+}\right\}$, then

$$
\Pi^{(q)} \equiv 0 \quad \text { and } \quad N^{(q)} \equiv A \quad \text { on } D,
$$

where $A$ is as in Theorem 3.1. If $q \in\left\{n_{-}, n_{+}\right\}$, then

$$
\Pi^{(q)} \equiv S_{-}+S_{+} \quad \text { and } \quad N^{(q)} \equiv A \quad \text { on } D,
$$

where $S_{-}, S_{+}$and $A$ are as in Theorem 3.2 . 
By using Corollary 1.6 and some standard argument in functional analysis, we can establish the spectral theory of $\square_{b}^{(q)}$ when the Levi form is non-degenerate of constant signature on $X$.

Proof of Theorem 1.7 Let $0<\mu<\mu_{1}<\infty$. We claim that $\operatorname{Spec} \square_{b}^{(q)} \cap\left[\mu, \mu_{1}\right]$ is a discrete subset of $\mathbb{R}$. We assume that Spec $\square_{b}^{(q)} \cap\left[\mu, \mu_{1}\right]$ is not a discrete subset of $\mathbb{R}$. Then, we can find $f_{j} \in E\left(\left[\mu, \mu_{1}\right]\right), j=1,2, \ldots$, with $\left(f_{j} \mid f_{\ell}\right)=\delta_{j, \ell}$, for all $j, \ell=1,2, \ldots$ Take $0<\lambda_{1}<\mu<\mu_{1}<$ $\lambda_{2}<\infty$. Then, we have

$$
f_{j}=\Pi_{\left(\lambda_{1}, \lambda_{2}\right]}^{(q)} f_{j}, \quad j=1,2, \ldots
$$

In view of Corollary 1.6, we know that $\Pi_{\left(\lambda_{1}, \lambda_{2}\right]}^{(q)}$ is a smoothing operator on $X$ and hence $\Pi_{\left(\lambda_{1}, \lambda_{2}\right]}^{(q)}$ is a compact operator on $L_{(0, q)}^{2}(X)$. By Rellich's theorem, we can find a subsequence $\left\{f_{s_{k}}\right\}_{k=1}^{\infty}$ of $\left\{f_{j}\right\}_{j=1}^{\infty}$, where $1<s_{1}<s_{2}<\ldots$, such that $f_{s_{k}} \rightarrow f$ in $L_{(0, q)}^{2}(X)$ as $k \rightarrow \infty$, for some $f \in L_{(0, q)}^{2}(X)$. But $\left(f_{s_{k}} \mid f_{s_{\ell}}\right)=0$ if $k \neq \ell$, we get a contradiction. We conclude that Spec $\square_{b}^{(q)} \cap\left[\mu, \mu_{1}\right]$ is a discrete subset of $\mathbb{R}$, for any $0<\mu<\mu_{1}<\infty$. Thus, for any $\mu>0$, Spec $\square_{b}^{(q)} \cap[\mu, \infty)$ is a discrete subset of $\mathbb{R}$.

Let $\nu \in \operatorname{Spec} \square_{b}^{(q)}$ with $\nu>0$. Since Spec $\square_{b}^{(q)} \cap\left[\mu, \mu_{1}\right]$ is discrete, where $0<\mu<\nu<\mu_{1}$, we have

$$
\square_{b}^{(q)}-\nu: \operatorname{Dom} \square_{b}^{(q)} \subset L_{(0, q)}^{2}(X) \rightarrow L_{(0, q)}^{2}(X)
$$

has $L^{2}$ closed range. Hence, if $\square_{b}^{(q)}-\nu$ is injective, then Range $\left(\square_{b}^{(q)}-\nu\right)=L_{(0, q)}^{2}(X)$ and $\square_{b}^{(q)}-\nu$ has a bounded inverse $\left(\square_{b}^{(q)}-\nu\right)^{-1}: L_{(0, q)}^{2}(X) \rightarrow L_{(0, q)}^{2}(X)$. Thus, $\nu$ is a resolvent if $\square_{b}^{(q)}-\nu$ is injective. We conclude that $\square_{b}^{(q)}-\nu$ is not injective, that is, $\nu$ is an eigenvalue of $\square_{b}^{(q)}$. Take $0<\lambda_{1}<\nu<\lambda_{2}<\infty$. We have

$$
H_{b, \nu}^{(q)}(X)=\Pi_{\left(\lambda_{1}, \lambda_{2}\right]}^{(q)} H_{b, \nu}^{(q)}(X)=\left\{\Pi_{\left(\lambda_{1}, \lambda_{2}\right]}^{(q)} f ; f \in H_{b, \nu}^{q}(X)\right\} .
$$

Since $\Pi_{\left(\lambda_{1}, \lambda_{2}\right]}^{(q)}$ is a smoothing operator on $X$, we conclude that $H_{b, \nu}^{(q)}(X) \subset \Omega^{0, q}(X)$. Moreover, from Rellich's theorem, we see that $\operatorname{dim} H_{b, \nu}^{(q)}(X)<\infty$. The theorem follows.

\section{SZEGŐ KERNEL ASYMPTOTIC EXPANSIONS}

In this section, we will apply Theorem 3.1 and Theorem 3.2 to establish Szegö kernel asymptotic expansions on the non-degenerate part of the Levi form under certain local conditions.

In view of Theorem 1.5, we see that if $\square_{b}^{(q)}$ has $L^{2}$ closed range, then $\Pi^{(q)}$ admits a full asymptotic expansion on the non-degenerate part of the Levi form. But in general, it is difficult to see that if $\square_{b}^{(q)}$ has $L^{2}$ closed range. We then impose the condition of local $L^{2}$ closed range, cf. Definition 1.8. It is clearly that if $\square_{b}^{(q)}$ has $L^{2}$ closed range then $\square_{b}^{(q)}$ has local $L^{2}$ closed range on every open set $D$ with respect to the identity map $I$.

We now prove the following precise version of Theorem 1.9 ,

Theorem 5.1. Let $X$ be a CR manifold of dimension $2 n-1$, whose Levi form is non-degenerate of constant signature $\left(n_{-}, n_{+}\right)$at each point of an open set $D \Subset X$. Let $q \in\{0,1, \ldots, n-1\}$ and let $Q \in L_{\mathrm{cl}}^{0}\left(X, T^{* 0, q} X \otimes T^{* 0, q} X\right)$ be a classical pseudodifferential operator on $X$ and let 
$Q^{*} \in L_{\mathrm{cl}}^{0}\left(X, T^{* 0, q} X \otimes T^{* 0, q} X\right)$ be the $L^{2}$ adjoint of $Q$ with respect to $(\cdot \mid \cdot)$. Suppose that $\square_{b}^{(q)}$ has local $L^{2}$ closed range on $D$ with respect to $Q$ and $Q \Pi^{(q)}=\Pi^{(q)} Q$ on $L_{(0, q)}^{2}(X)$. Then,

$$
Q^{*} \Pi^{(q)} Q \equiv 0 \text { on } D \text { if } q \notin\left\{n_{-}, n_{+}\right\}
$$

and if $q \in\left\{n_{-}, n_{+}\right\}$, then

$$
\left(Q^{*} \Pi^{(q)} Q\right)(x, y) \equiv \int_{0}^{\infty} e^{i \varphi_{-}(x, y) t} a_{-}(x, y, t) d t+\int_{0}^{\infty} e^{i \varphi_{+}(x, y) t} a_{+}(x, y, t) d t \text { on } D,
$$

where $\varphi_{ \pm}(x, y) \in \mathscr{C}^{\infty}(D \times D)$ are as in Theorem 4.1 and $a_{-}(x, y, t), a_{+}(x, y, t) \in S_{\mathrm{cl}}^{n-1}(D \times D \times$ $\left.\mathbb{R}_{+}, T_{y}^{* 0, q} X \otimes T_{x}^{* 0, q} X\right)$ satisfy

$$
\begin{aligned}
& a_{-}(x, y, t)=0 \text { if } q \neq n_{-} \text {or } Q \equiv 0 \text { at } \Sigma^{-} \cap T^{*} D, \\
& a_{+}(x, y, t)=0 \text { if } q \neq n_{+} \text {or } Q \equiv 0 \text { at } \Sigma^{+} \cap T^{*} D .
\end{aligned}
$$

(See Definition 2.4 for the meaning of $Q \equiv 0$ at $\Sigma^{-} \bigcap T^{*} D$.) Moreover, assume that $q=n_{-}$, then the leading term $a_{-}^{0}(x, y)$ of the expansion (2.16) of $a_{-}(x, y, t)$ satisfies

$$
a_{-}^{0}(x, x)=\frac{1}{2} \pi^{-n} \frac{v(x)}{m(x)}\left|\operatorname{det} \mathcal{L}_{x}\right| \tau_{x, n_{-}} q^{*}\left(x,-\omega_{0}(x)\right) q\left(x,-\omega_{0}(x)\right) \tau_{x, n_{-}}, \quad \forall x \in D,
$$

where det $\mathcal{L}_{x}$ is the determinant of the Levi form defined in (3.7), $v(x)$ is the volume form on $X$ induced by $\langle\cdot \mid \cdot\rangle, q(x, \eta) \in \mathscr{C}^{\infty}\left(T^{*} D\right)$ is the principal symbol of $Q, q^{*}(x, \eta)$ is the adjoint of $q(x, \eta): T_{x}^{* 0, q} X \rightarrow T_{x}^{* 0, q} X$ with respect to $\langle\cdot \mid \cdot\rangle$ and $\tau_{x, n_{-}}$is as in (3.9).

To prove Theorem 5.1 we need a series of results, starting with the following.

Theorem 5.2. In the conditions of Theorem 5.1 we have (5.1) and

$$
Q^{*} \Pi^{(q)} Q \equiv\left(S_{-}^{*}+S_{+}^{*}\right) Q^{*} Q\left(S_{-}+S_{+}\right) \text {on } D \text { if } q \in\left\{n_{-}, n_{+}\right\},
$$

where $S_{-}$and $S_{+}$are as in Theorem 3.2

Proof. We first assume that $q \in\left\{n_{-}, n_{+}\right\}$. Put $S=S_{-}+S_{+}$, where $S_{-}$and $S_{+}$are as in Theorem 3.2 and let $S^{*}$ be the adjoint of $S$. From (3.1), we have

$$
\Pi^{(q)}=\left(A^{*} \square_{b}^{(q)}+S^{*}\right) \Pi^{(q)}=S^{*} \Pi^{(q)}
$$

and hence

$$
\Pi^{(q)}=\Pi^{(q)} S \text { on } D .
$$

Fix $D^{\prime} \Subset D$. Let $u \in \Omega_{0}^{0, q}\left(D^{\prime}\right)$. Since $\square_{b}^{(q)}$ has local $L^{2}$ closed range on $D$ with respect to $Q$, we have for every $s \in \mathbb{Z}$,

$$
\left\|Q\left(I-\Pi^{(q)}\right) S u\right\| \leq C_{D^{\prime}, s} \sqrt{\left\|\left(\square_{b}^{(q)}\right)^{p} S u\right\|_{s}\|u\|_{-s}}, \quad \forall u \in \Omega_{0}^{0, q}\left(D^{\prime}\right),
$$

where $C_{D^{\prime}, s}>0, p \in \mathbb{N}$ are constants independent of $u$ and $\|\cdot\|_{s}$ denotes the usual Sobolev norm of order $s$ on $D^{\prime}$. Since $\left(\square_{b}^{(q)}\right)^{p} S \equiv 0$ on $D$, for every $s \in \mathbb{N}_{0}$, there is a constant $C_{s}>0$ such that

$$
\left\|\left(\square_{b}^{(q)}\right)^{p} S u\right\|_{s} \leq C_{s}\|u\|_{-s}, \quad \forall u \in \Omega_{0}^{0, q}\left(D^{\prime}\right) .
$$

From (5.9) and (5.8), we can extend $Q\left(I-\Pi^{(q)}\right) S=Q S-Q \Pi^{(q)}$ (here we used (5.7)) to $H_{\text {comp }}^{-s}\left(D, T^{* 0, q} X\right), \forall s \in \mathbb{N}_{0}$ and we have

$$
Q S-Q \Pi^{(q)}: H_{\text {comp }}^{-s}\left(D, T^{* 0, q} X\right) \rightarrow L_{(0, q)}^{2}(X) \text { is continuous, } \forall s \in \mathbb{N}_{0} \text {. }
$$


By taking adjoint in (5.10), we get

$$
S^{*} Q^{*}-\Pi^{(q)} Q^{*}: L_{(0, q)}^{2}(X) \rightarrow H_{\mathrm{loc}}^{s}\left(D, T^{* 0, q} X\right) \text { is continuous, } \forall s \in \mathbb{N}_{0} \text {. }
$$

From (5.10) and (5.11), we conclude that for any $s \in \mathbb{N}_{0}$ the map

$$
\left(S^{*} Q^{*}-\Pi^{(q)} Q^{*}\right)\left(Q S-Q \Pi^{(q)}\right): H_{\text {comp }}^{-s}\left(D, T^{* 0, q} X\right) \rightarrow H_{\text {loc }}^{s}\left(D, T^{* 0, q} X\right) .
$$

is continuous. Hence,

$$
\left(S^{*} Q^{*}-\Pi^{(q)} Q^{*}\right)\left(Q S-Q \Pi^{(q)}\right) \equiv 0 \text { on } D .
$$

Now,

$$
\begin{aligned}
& \left(S^{*} Q^{*}-\Pi^{(q)} Q^{*}\right)\left(Q S-Q \Pi^{(q)}\right) \\
& =S^{*} Q^{*} Q S-S^{*} Q^{*} Q \Pi^{(q)}-\Pi^{(q)} Q^{*} Q S+\Pi^{(q)} Q^{*} Q \Pi^{(q)} \\
& =S^{*} Q^{*} Q S-S^{*} \Pi^{(q)} Q^{*} Q-Q^{*} Q \Pi^{(q)} S+Q^{*} Q \Pi^{(q)} \\
& =S^{*} Q^{*} Q S-\Pi^{(q)} Q^{*} Q \Pi^{(q)} .
\end{aligned}
$$

Here we used $Q \Pi^{(q)}=\Pi^{(q)} Q, Q^{*} \Pi^{(q)}=\Pi^{(q)} Q^{*}$, (5.6) and (5.7). From (5.13) and (5.12), (5.5) follows. By using Theorem 3.1, we can repeat the procedure above and obtain (5.1). We omit the details.

In the rest of this section, we will study the kernel $\left(S_{-}^{*}+S_{+}^{*}\right) Q^{*} Q\left(S_{-}+S_{+}\right)(x, y)$. We will use the notations and assumptions used in Theorem 5.2 and until further notice we assume that $q=n_{-}$. Let $\varphi_{-}(x, y) \in \mathscr{C}^{\infty}(D \times D), \varphi_{+}(x, y) \in \mathscr{C}^{\infty}(D \times D)$ be as in Theorem 3.2. We need the following result, which is essentially well-known and follows from the stationary phase formula of Melin-Sjöstrand [60] (see also [44, p. 76-77] for more details).

Lemma 5.3. There is a complex valued phase function $\varphi \in \mathscr{C}^{\infty}(D \times D)$ with $\varphi(x, x)=0$, $\left.d_{x} \varphi(x, y)\right|_{x=y}=-\omega_{0}(x),\left.d_{y} \varphi(x, y)\right|_{x=y}=\omega_{0}(x)$ and $\varphi(x, y)$ satisfies (3.4) such that for any properly supported operators $B, C: \mathscr{D}^{\prime}\left(D, T^{* 0, q} X\right) \rightarrow \mathscr{D}^{\prime}\left(D, T^{* 0, q} X\right)$,

$$
B=\int_{0}^{\infty} e^{i \varphi_{-}(x, y) t} b(x, y, t) d t, \quad C=\int_{0}^{\infty} e^{i \varphi_{-}(x, y) t} c(x, y, t) d t,
$$

with $b(x, y, t), c(x, y, t) \in S_{\mathrm{cl}}^{n-1}\left(D \times D \times \mathbb{R}_{+}, T^{* 0, q} X \otimes T^{* 0, q} X\right)$, we have

$$
B \circ C \equiv e^{i \varphi(x, y) t} d(x, y, t) d t \text { on } D,
$$

where $d(x, y, t) \in S_{\mathrm{cl}}^{n-1}\left(D \times D \times \mathbb{R}_{+}, T^{* 0, q} X \otimes T^{* 0, q} X\right)$ and the leading term $d_{0}(x, y)$ of the expansion (2.16) of $d(x, y, t)$ satisfies

$$
d_{0}(x, x)=2 \pi^{n} \frac{m(x)}{v(x)}\left|\operatorname{det} \mathcal{L}_{x}\right|^{-1} b_{0}(x, x) c_{0}(x, x), \quad \forall x \in D,
$$

where $b_{0}(x, y), c_{0}(x, y)$ denote the leading terms of the expansions (2.16) of $b(x, y, t), c(x, y, t)$ respectively.

We postpone the proof of the following Theorem for Section 8 ,

Theorem 5.4. With the notations and assumptions used above, there is a $g(x, y) \in \mathscr{C}^{\infty}(D \times D)$ with $g(x, x)=1$ such that

$$
\varphi(x, y)-g(x, y) \varphi_{-}(x, y) \text { vanishes to infinite order at } x=y \text {. }
$$

From Lemma 5.3 and Theorem 5.4 , we deduce 
Corollary 5.5. In the conditions of Lemma 5.3 we have

$$
B \circ C \equiv e^{i \varphi_{-}(x, y) t} e(x, y, t) d t \text { on } D,
$$

where $e(x, y, t) \in S_{\mathrm{cl}}^{n-1}\left(D \times D \times \mathbb{R}_{+}, T^{* 0, q} X \otimes T^{* 0, q} X\right)$ and the leading term $e_{0}(x, y)$ of the expansion (2.16) of $e(x, y, t)$ satisfies (5.14).

Similarly, we can repeat the proof of Corollary 5.5 and conclude that

Theorem 5.6. With the notations and assumptions used in Theorem 5.2, let $q=n_{+}$. For any properly supported operators $\mathcal{B}, \mathcal{C}: \mathscr{D}^{\prime}\left(D, T^{* 0, q} X\right) \rightarrow \mathscr{D}^{\prime}\left(D, T^{* 0, q} X\right)$,

$$
\mathcal{B}=\int_{0}^{\infty} e^{i \varphi_{+}(x, y) t} b(x, y, t) d t, \quad \mathcal{C}=\int_{0}^{\infty} e^{i \varphi_{+}(x, y) t} c(x, y, t) d t
$$

with $b(x, y, t), c(x, y, t) \in S_{\mathrm{cl}}^{n-1}\left(D \times D \times \mathbb{R}_{+}, T^{* 0, q} X \otimes T^{* 0, q} X\right)$, we have

$$
\mathcal{B} \circ \mathcal{C} \equiv e^{i \varphi_{+}(x, y) t} f(x, y, t) d t \text { on } D,
$$

where $f(x, y, t) \in S_{\mathrm{cl}}^{n-1}\left(D \times D \times \mathbb{R}_{+}, T^{* 0, q} X \otimes T^{* 0, q} X\right)$ and the leading term $f_{0}(x, y)$ of the expansion (2.16) of $f(x, y, t)$ satisfies (5.14).

We also need the following.

Lemma 5.7. With the notations and assumptions used in Theorem 5.2] let $q=n_{+}=n_{-}$. For any properly supported operators $\mathcal{B}, C: \mathscr{D}^{\prime}\left(D, T^{* 0, q} X\right) \rightarrow \mathscr{D}^{\prime}\left(D, T^{* 0, q} X\right)$,

$$
\mathcal{B}=\int_{0}^{\infty} e^{i \varphi_{+}(x, y) t} b(x, y, t) d t, \quad C=\int_{0}^{\infty} e^{i \varphi_{-}(x, y) t} c(x, y, t) d t,
$$

where $b(x, y, t), c(x, y, t) \in S_{\mathrm{cl}}^{n-1}\left(D \times D \times \mathbb{R}_{+}, T^{* 0, q} X \otimes T^{* 0, q} X\right)$, we have

$$
\mathcal{B} \circ C \equiv 0 \text { and } C \circ \mathcal{B} \equiv 0 \text { on } D \text {. }
$$

Proof. We first notice that $\mathcal{B} \circ C$ is smoothing away $x=y$. We also write $w=\left(w_{1}, \ldots, w_{2 n-1}\right)$ to denote local coordinates on $D$. We have

$$
\begin{aligned}
\mathcal{B} \circ C(x, y) & =\int_{\sigma>0, t>0} e^{i \varphi_{+}(x, w) \sigma+i \varphi_{-}(w, y) t} b(x, w, \sigma) c(w, y, t) d \sigma m(w) d t \\
& =\int_{s>0, t>0} e^{i t\left(\varphi_{+}(x, w) s+\varphi_{-}(w, y)\right)} t b(x, w, s t) c(w, y, t) d s m(w) d t .
\end{aligned}
$$

Take $\chi \in \mathscr{C}_{0}^{\infty}(\mathbb{R},[0,1])$ with $\chi=1$ on $\left[-\frac{1}{2}, \frac{1}{2}\right], \chi=0$ on $\left.]-\infty,-1\right] \bigcup[1, \infty[$. From (5.16), we have

$$
\begin{aligned}
& \mathcal{B} \circ C(x, y)=I_{\varepsilon}+I I_{\varepsilon}, \\
& I_{\varepsilon}=\int_{s>0, t>0} e^{i t\left(\varphi_{+}(x, w) s+\varphi_{-}(w, y)\right)} \chi\left(\frac{|x-w|^{2}}{\varepsilon}\right) t b(x, w, s t) c(w, y, t) d s m(w) d t, \\
& I I_{\varepsilon}=\int_{s>0, t>0} e^{i t\left(\varphi_{+}(x, w) s+\varphi_{-}(w, y)\right)}\left(1-\chi\left(\frac{|x-w|^{2}}{\varepsilon}\right)\right) \\
& \quad \quad \times t b(x, w, s t) c(w, y, t) d s m(w) d t,
\end{aligned}
$$

where $\varepsilon>0$ is a small constant. Since $\varphi_{+}(x, w)=0$ if and only if $x=w$, we can integrate by parts with respect to $s$ and conclude that $I I_{\varepsilon}$ is smoothing. Since $\mathcal{B} \circ C$ is smoothing away $x=$ $y$, we may assume that $|x-y|<\varepsilon$. Since $\left.d_{w}\left(\varphi_{+}(x, w) s+\varphi_{-}(w, y)\right)\right|_{x=y=w}=-\omega_{0}(x)(s+1) \neq 0$, if $\varepsilon>0$ is small, we can integrate by parts with respect to $w$ and conclude that $I_{\varepsilon} \equiv 0$ on 
$D$. We get $\mathcal{B} \circ C \equiv 0$ on $D$. Similarly, we can repeat the procedure above and conclude that $C \circ \mathcal{B} \equiv 0$ on $D$. The lemma follows.

Recalling Definition 2.4 we see that Theorem 5.5, Theorem 5.6 and Lemma 5.7yield:

Theorem 5.8. With the notations and assumptions used in Theorem 5.2 let $q \in\left\{n_{-}, n_{+}\right\}$. Then,

$$
\begin{aligned}
& \left(S_{-}^{*}+S_{+}^{*}\right) Q^{*} Q\left(S_{-}+S_{+}\right)(x, y) \\
& \equiv \int_{0}^{\infty} e^{i \varphi_{-}(x, y) t} a_{-}(x, y, t) d t+\int_{0}^{\infty} e^{i \varphi_{+}(x, y) t} a_{+}(x, y, t) d t \text { on } D,
\end{aligned}
$$

where $\varphi_{ \pm}(x, y) \in \mathscr{C}^{\infty}(D \times D)$ are as in Theorem 4.1. $a_{ \pm}(x, y, t) \in S_{\mathrm{cl}}^{n-1}\left(D \times D \times \mathbb{R}_{+}, T_{y}^{* 0, q} X \otimes\right.$ $\left.T_{x}^{* 0, q} X\right)$,

$$
\begin{aligned}
& a_{-}(x, y, t)=0 \text { if } q \neq n_{-} \text {or } Q \equiv 0 \text { at } \Sigma^{-} \cap T^{*} D, \\
& a_{+}(x, y, t)=0 \text { if } q \neq n_{+} \text {or } Q \equiv 0 \text { at } \Sigma^{+} \cap T^{*} D .
\end{aligned}
$$

Moreover, assume that $q=n_{-}$, then, for the leading term $a_{-}^{0}(x, y)$ of the expansion (2.16) of $a_{-}(x, y, t)$ satisfies

$$
a_{-}^{0}(x, x)=\frac{1}{2} \pi^{-n} \frac{v(x)}{m(x)}\left|\operatorname{det} \mathcal{L}_{x}\right| \tau_{x, n_{-}} q^{*}\left(x,-\omega_{0}(x)\right) q\left(x,-\omega_{0}(x)\right) \tau_{x, n_{-}}, \quad \forall x \in D,
$$

where $\operatorname{det} \mathcal{L}_{x}$ is the determinant of the Levi form defined in (3.7), $v(x)$ is the volume form on $X$ induced by $\langle\cdot \mid \cdot\rangle, q(x, \eta) \in \mathscr{C}^{\infty}\left(T^{*} D\right)$ is the principal symbol of $Q, q^{*}(x, \eta)$ is the adjoint of $q(x, \eta): T_{x}^{* 0, q} X \rightarrow T_{x}^{* 0, q} X$ with respect to $\langle\cdot \mid \cdot\rangle$ and $\tau_{x, n_{-}}$is as in (3.9).

Proof of Theorem 1.9 From Theorem 5.2 and Theorem 5.8, we get Theorem 5.1 and Theorem 1.9 .

Proof of Theorem 1.10. Fix $p \in D$, let $\left\{W_{j}\right\}_{j=1}^{n-1}$ be an orthonormal frame of $T^{1,0} X$ in a neighbourhood of $p$ such that the Levi form is diagonal at $p$. We take local coordinates $x=$ $\left(x_{1}, \ldots, x_{2 n-1}\right), z_{j}=x_{2 j-1}+i x_{2 j}, j=1, \ldots, n-1$, defined on some neighbourhood of $p$ such that $\omega_{0}\left(x_{0}\right)=d x_{2 n-1}, x(p)=0$, and for some $c_{j} \in \mathbb{C}, j=1, \ldots, n-1$,

$$
W_{j}=\frac{\partial}{\partial z_{j}}-i \mu_{j} \bar{z}_{j} \frac{\partial}{\partial x_{2 n-1}}-c_{j} x_{2 n-1} \frac{\partial}{\partial x_{2 n-1}}+O\left(|x|^{2}\right), j=1, \ldots, n-1 .
$$

For $x=\left(x_{1}, x_{2}, \ldots, x_{2 n-1}\right)$, we write $x^{\prime}=\left(x_{1}, x_{2}, \ldots, x_{2 n-2}\right)$. Take $\chi \in \mathscr{C}_{0}^{\infty}(]-\varepsilon_{0}, \varepsilon_{0}[), \chi=1$ near $0, \chi(t)=\chi(-t)$, where $\varepsilon_{0}>0$ is a small constant. Take $\varepsilon_{0}>0$ small enough so that $\left.D^{\prime} \times\right]-\varepsilon_{0}, \varepsilon_{0}\left[\Subset D\right.$, where $D^{\prime}$ is an open neighbourhood of $0 \in \mathbb{R}^{2 n-2}$. For each $k>0$, we consider the operator

$$
E_{k}: u \in \mathscr{C}_{0}^{\infty}\left(D^{\prime}\right) \rightarrow\left(Q^{*} \Pi^{(0)} Q\right)\left(e^{-i k y_{2 n-1}} \chi\left(y_{2 n-1}\right) u\left(y^{\prime}\right)\right) \in \mathscr{C}^{\infty}(X) .
$$

From the stationary phase formula of Melin-Sjöstrand [60], we can check that $E_{k}$ is smoothing and the kernel of $E_{k}$ satisfies

$$
E_{k}\left(x, y^{\prime}\right) \equiv e^{i k \Phi\left(x, y^{\prime}\right)} g\left(x, y^{\prime}, k\right) \quad \bmod O\left(k^{-\infty}\right),
$$


where $g\left(x, y^{\prime}, k\right) \in \mathscr{C}^{\infty}, g\left(x, y^{\prime}, k\right) \sim \sum_{j=0}^{\infty} g_{j}\left(x, y^{\prime}\right) k^{n-1-j}$ in $S_{\operatorname{loc}}^{n-1}(1), g_{j}\left(x, y^{\prime}\right) \in \mathscr{C}^{\infty}, j=$ $0,1,2, \ldots, g_{0}\left(x, x^{\prime}\right) \neq 0, \Phi \in \mathscr{C}^{\infty}, \operatorname{Im} \Phi \geq 0, \Phi\left(x, x^{\prime}\right)=0$ and

$$
\begin{aligned}
& \Phi\left(x, y^{\prime}\right)=-x_{2 n-1}+i \sum_{j=1}^{n-1} \mu_{j}\left|z_{j}-w_{j}\right|^{2} \\
& \quad+\sum_{j=1}^{n-1}\left(i \mu_{j}\left(\bar{z}_{j} w_{j}-z_{j} \bar{w}_{j}\right)-c_{j} z_{j} x_{2 n-1}-\bar{c}_{j} \bar{z}_{j} x_{2 n-1}\right) \\
& \quad+x_{2 n-1} f\left(x, y^{\prime}\right)+O\left(\left|\left(x, y^{\prime}\right)\right|^{3}\right), \\
& \quad f \in \mathscr{C}^{\infty}, \quad f(0,0)=0, \quad w_{j}=y_{2 j-1}+i y_{2 j}, \quad j=1, \ldots, n-1 .
\end{aligned}
$$

(See Section 8 for the details and the precise meanings of $A \equiv B \bmod O\left(k^{-\infty}\right)$ and $S_{\text {loc }}^{n-1}(1)$.) Put

$$
u_{k}(x):=E_{k}\left(\chi\left(k y_{1}\right) \chi\left(k y_{2}\right) \ldots \chi\left(k y_{2 n-2}\right) k^{2 n-2}\right) .
$$

Then $u_{k}(x)$ is a global smooth CR function on $X$. From (5.21) and (5.22), we can check that

$$
\begin{aligned}
\lim _{k \rightarrow \infty} k^{-n} \frac{\partial u_{k}}{\partial x_{2 n-1}}(0) & =\lim _{k \rightarrow \infty} k^{-n} \int e^{i k \Phi\left(0, y^{\prime}\right)}(-i k) g\left(0, y^{\prime}, k\right) \chi\left(k y_{1}\right) \ldots \chi\left(k y_{2 n-2}\right) k^{2 n-2} d y^{\prime} \\
& =(-i) g_{0}(0,0) \int \chi\left(y_{1}\right) \ldots \chi\left(y_{2 n-2}\right) d y^{\prime} \\
\lim _{k \rightarrow \infty} k^{-n} \frac{\partial u_{k}}{\partial x_{t}}(0) & =0, \quad t=1,2, \ldots, 2 n-2 .
\end{aligned}
$$

For any $s \in\{1,2, \ldots, n-1\}$, put

$$
u_{k}^{s}(x):=E_{k}\left(k\left(y_{2 s-1}+i y_{2 s}\right) \chi\left(k y_{1}\right) \chi\left(k y_{2}\right) \ldots \chi\left(k y_{2 n-2}\right) k^{2 n-2}\right) .
$$

Then $u_{k}^{s}(x)$ is a global smooth CR function on $X, s=1,2, \ldots, n-1$. From (5.21), (5.22) and notice that $\bar{\partial}_{b} u_{k}^{s}=0, s=1,2, \ldots, n-1$, we can check that

$$
\begin{aligned}
& \lim _{k \rightarrow \infty} k^{-n+1} \frac{\partial u_{k}^{s}}{\partial z_{s}}(0) \\
& =\lim _{k \rightarrow \infty} k^{-n+1} \int e^{i k \Phi\left(0, y^{\prime}\right)} 2 k^{2} \mu_{s}\left|y_{2 s-1}+i y_{2 s}\right|^{2} g\left(0, y^{\prime}, k\right) \chi\left(k y_{1}\right) \ldots \chi\left(k y_{2 n-2}\right) k^{2 n-2} d y^{\prime} \\
& =2 \mu_{s} g_{0}(0,0) \int\left|y_{2 s-1}+i y_{2 s}\right|^{2} \chi\left(y_{1}\right) \ldots \chi\left(y_{2 n-2}\right) d y^{\prime} \\
& \lim _{k \rightarrow \infty} k^{-n+1} \frac{\partial u_{k}^{s}}{\partial \bar{z}_{t}}(0)=0, \quad t=1,2, \ldots, n-1
\end{aligned}
$$

and for $t \neq s, t \in\{1,2, \ldots, n-1\}$, we have

$$
\begin{aligned}
& \lim _{k \rightarrow \infty} k^{-n+1} \frac{\partial u_{k}^{s}}{\partial z_{t}}(0) \\
& =\lim _{k \rightarrow \infty} k^{-n+1} \int e^{i k \Phi\left(0, y^{\prime}\right)} 2 k^{2} \mu_{t}\left(y_{2 t-1}-i y_{2 t}\right)\left(y_{2 s-1}+i y_{2 s}\right) g\left(0, y^{\prime}, k\right) \chi\left(k y_{1}\right) \ldots \chi\left(k y_{2 n-2}\right) k^{2 n-2} d y^{\prime} \\
& =2 \mu_{t} g_{0}(0,0) \int\left(y_{2 t-1}-i y_{2 t}\right)\left(y_{2 s-1}+i y_{2 s}\right) \chi\left(y_{1}\right) \ldots \chi\left(y_{2 n-2}\right) d y^{\prime}=0 .
\end{aligned}
$$


From (5.24), (5.26) and (5.27), it is not difficult to check that for $k$ large, the differential of the CR map

$$
x \in X \rightarrow\left(u_{k}(x), u_{k}^{1}(x), \ldots, u_{k}^{n-1}(x)\right) \in \mathbb{C}^{n}
$$

is injective at $p$. Thus, near $p$, the map $x \in X \rightarrow\left(u_{k}(x), u_{k}^{1}(x), \ldots, u_{k}^{n-1}(x)\right) \in \mathbb{C}^{n}$ is a CR embedding. Theorem 1.10 follows.

\section{SZEGŐ PROJECTIONS ON CR MANIFOLDS WITH TRANSVERSAL CR $S^{1}$ ACTIONS}

In this section, we will apply Theorem 1.9 to establish Szegő kernel asymptotic expansions on compact CR manifolds with transversal CR $S^{1}$ actions under certain Levi curvature assumptions. As an application, we will show that if $X$ is a 3-dimensional compact strictly pseudoconvex CR manifold with a transversal CR $S^{1}$ action, then $X$ can be CR embedded into $\mathbb{C}^{N}$, for some $N \in \mathbb{N}$. We introduce some notations first.

Let $\left(X, T^{1,0} X\right)$ be a CR manifold. Let assume that $X$ admits a $S^{1}$ action $S^{1} \times X \rightarrow X$, $\left(e^{i \theta}, x\right) \mapsto e^{i \theta} x$. Let $T \in \mathscr{C}^{\infty}(X, T X)$ be the real vector field given by

$$
T u=\left.\frac{\partial}{\partial \theta} u\left(e^{i \theta} x\right)\right|_{\theta=0}, u \in \mathscr{C}^{\infty}(X) .
$$

We call $T$ the global vector field induced by the $S^{1}$ action or the infinitesimal generator of the action.

Definition 6.1. We say that the $S^{1}$ action $e^{i \theta}$ is CR if

$$
\left[T, \mathscr{C}^{\infty}\left(X, T^{1,0} X\right)\right] \subset \mathscr{C}^{\infty}\left(X, T^{1,0} X\right)
$$

and is transversal if for every point $x \in X$,

$$
T(x) \oplus T_{x}^{1,0} X \oplus T_{x}^{0,1} X=\mathbb{C} T_{x} X .
$$

Until further notice, we assume that $\left(X, T^{1,0} X\right)$ is a CR manifold with a transversal CR $S^{1}$ action and we let $T$ be the global vector field induced by the $S^{1}$ action.

Fix $\theta_{0} \in\left[0,2 \pi\left[\right.\right.$. Let $d e^{i \theta_{0}}: \mathbb{C} T_{x} X \rightarrow \mathbb{C} T_{e^{i \theta_{0}}} X$ denote the differential of the map $e^{i \theta_{0}}: X \rightarrow$ $X$.

Definition 6.2. Let $U \subset X$ be an open set and let $V \in \mathscr{C}^{\infty}(U, \mathbb{C} T X)$ be a vector field on $U$. We say that $V$ is $T$-rigid if $d e^{i \theta_{0}} V(x)=V(x), \forall x \in e^{i \theta_{0}} U \cap U$, for every $\theta_{0} \in[0,2 \pi$ [ with $e^{i \theta_{0}} U \cap U \neq \emptyset$.

We also need

Definition 6.3. Let $\langle\cdot \mid \cdot\rangle$ be a Hermitian metric on $\mathbb{C} T X$. We say that $\langle\cdot \mid \cdot\rangle$ is $T$-rigid if for $T$-rigid vector fields $V$ and $W$ on $U$, where $U \subset X$ is any open set, we have

$$
\langle V(x) \mid W(x)\rangle=\left\langle d e^{i \theta_{0}} V\left(e^{i \theta_{0}} x\right) \mid d e^{i \theta_{0}} W\left(e^{i \theta_{0}} x\right)\right\rangle, \quad \forall x \in U, \theta_{0} \in[0,2 \pi[.
$$

The following result was established in [46, Theorem 9.2].

Theorem 6.4. There is a T-rigid Hermitian metric $\langle\cdot \mid \cdot\rangle$ on $\mathbb{C} T X$ such that $T^{1,0} X \perp T^{0,1} X$, $T \perp\left(T^{1,0} X \oplus T^{0,1} X\right),\langle T \mid T\rangle=1$ and $\langle u \mid v\rangle$ is real if $u, v$ are real tangent vectors.

Until further notice, we fix a $T$-rigid Hermitian metric $\langle\cdot \mid \cdot\rangle$ on $\mathbb{C} T X$ such that $T^{1,0} X \perp$ $T^{0,1} X, T \perp\left(T^{1,0} X \oplus T^{0,1} X\right),\langle T \mid T\rangle=1$ and $\langle u \mid v\rangle$ is real if $u, v$ are real tangent vectors and we take $m(x)$ to be the volume form induced by the given T-rigid Hermitian metric $\langle\cdot \mid \cdot\rangle$. We will use the same notations as before. We need the following result due to BaouendiRothschild-Treves [3, Section1] 
Theorem 6.5. For every point $x_{0} \in X$, there exists local coordinates $x=\left(x_{1}, \ldots, x_{2 n-1}\right)=$ $(z, \theta)=\left(z_{1}, \ldots, z_{n-1}, \theta\right), z_{j}=x_{2 j-1}+i x_{2 j}, j=1, \ldots, n-1, \theta=x_{2 n-1}$, defined in some small neighbourhood $U$ of $x_{0}$ such that

$$
T=\frac{\partial}{\partial \theta}, Z_{j}=\frac{\partial}{\partial z_{j}}+i \frac{\partial \phi}{\partial z_{j}}(z) \frac{\partial}{\partial \theta}, \quad j=1, \ldots, n-1,
$$

where $Z_{j}(x), j=1, \ldots, n-1$, form a basis of $T_{x}^{1,0} X$, for each $x \in U$, and $\phi(z) \in \mathscr{C}^{\infty}(U, \mathbb{R})$ is independent of $\theta$.

Let $x=\left(x_{1}, \ldots, x_{2 n-1}\right)=(z, \theta)=\left(z_{1}, \ldots, z_{n-1}, \theta\right), z_{j}=x_{2 j-1}+i x_{2 j}, j=1, \ldots, n-1$, $\theta=x_{2 n-1}$, be canonical coordinates of $X$ defined in some open set $D \Subset X$. It is clearly that

$$
\left\{d \bar{z}_{j_{1}} \wedge \ldots \wedge d \bar{z}_{j_{q}} ; 1 \leq j_{1}<j_{2}<\ldots<j_{q} \leq n-1\right\}
$$

is a basis for $T_{x}^{* 0, q} X$, for every $x \in D$. Let $u \in \Omega^{0, q}(X)$. On $D$, we write

$$
u=\sum_{1 \leq j_{1}<j_{2}<\ldots<j_{q} \leq n-1} u_{j_{1}, \ldots, j_{q}} d \bar{z}_{j_{1}} \wedge \ldots \wedge d \bar{z}_{j_{q}}, u_{j_{1}, \ldots, j_{q}} \in \mathscr{C}^{\infty}(D) .
$$

On $D$, we define

$$
T u:=\sum_{1 \leq j_{1}<j_{2}<\ldots<j_{q} \leq n-1}\left(T u_{j_{1}, \ldots, j_{q}}\right) d \bar{z}_{j_{1}} \wedge \ldots \wedge d \bar{z}_{j_{q}}
$$

Let $y=\left(y_{1}, \ldots, y_{2 n-1}\right)=(w, \gamma), w_{j}=y_{2 j-1}+i y_{2 j}, j=1, \ldots, n-1, \gamma=y_{2 n-1}$, be another canonical coordinates on $D$. Then,

$$
T=\frac{\partial}{\partial \gamma}, \widetilde{Z}_{j}=\frac{\partial}{\partial w_{j}}+i \frac{\partial \widetilde{\phi}}{\partial w_{j}}(w) \frac{\partial}{\partial \gamma}, j=1, \ldots, n-1,
$$

where $\widetilde{Z}_{j}(y), j=1, \ldots, n-1$, form a basis of $T_{y}^{1,0} X$, for each $y \in D$, and $\widetilde{\phi}(w) \in \mathscr{C}^{\infty}(D, \mathbb{R})$ independent of $\gamma$. From (6.4) and (6.2), it is not difficult to see that on $D$, we have

$$
\begin{aligned}
& w=\left(w_{1}, \ldots, w_{n-1}\right)=\left(H_{1}(z), \ldots, H_{n-1}(z)\right)=H(z), H_{j}(z) \in \mathscr{C}^{\infty}, \forall j, \\
& \gamma=\theta+G(z), \quad G(z) \in \mathscr{C}^{\infty},
\end{aligned}
$$

where for each $j=1, \ldots, n-1, H_{j}(z)$ is holomorphic. From (6.5), we can check that

$$
d \bar{w}_{j}=\sum_{l=1}^{n-1} \frac{\overline{\partial H_{j}}}{\partial z_{l}} d \bar{z}_{l}, \quad j=1, \ldots, n-1 .
$$

From (6.6), it is straightforward to check that the definition (6.3) is independent of the choice of canonical coordinates. We omit the details (see also [45, Section 5]). Thus, Tu is well-defined as an element in $\Omega^{0, q}(X)$.

For $m \in \mathbb{Z}$, put

$$
B_{m}^{0, q}(X):=\left\{u \in \Omega^{0, q}(X) ; T u=-i m u\right\}
$$

and let $\mathcal{B}_{m}^{0, q}(X) \subset L_{(0, q)}^{2}(X)$ be the completion of $B_{m}^{0, q}(X)$ with respect to $(\cdot \mid \cdot)$. It is easy to see that for any $m, m^{\prime} \in \mathbb{Z}, m \neq m^{\prime}$,

$$
(u \mid v)=0, \quad \forall u \in \mathcal{B}_{m}^{0, q}(X), v \in \mathcal{B}_{m^{\prime}}^{0, q}(X) .
$$

We have actually an orthogonal decomposition of Hilbert spaces

$$
L_{(0, q)}^{2}(X)=\widehat{\bigoplus}_{m \in \mathbb{Z}} \mathcal{B}_{m}^{0, q}(X) .
$$


For $m \in \mathbb{Z}$, let

$$
Q_{m}^{(q)}: L_{(0, q)}^{2}(X) \rightarrow \mathcal{B}_{m}^{0, q}(X)
$$

be the orthogonal projection with respect to $(\cdot \mid \cdot)$. Moreover, it is not difficult to see that for every $m \in \mathbb{Z}$, we have

$$
\begin{aligned}
& Q_{m}^{(q)}: \Omega^{0, q}(X) \rightarrow B_{m}^{0, q}(X), \\
& T Q_{m}^{(q)}=-i m Q_{m}^{(q)} u, \quad \forall u \in L_{(0, q)}^{2}(X), \\
& \left\|T Q_{m}^{(q)} u\right\|=|m|\left\|Q_{m}^{(q)} u\right\|, \quad \forall u \in L_{(0, q)}^{2}(X) .
\end{aligned}
$$

Since the Hermitian metric $\langle\cdot \mid \cdot\rangle$ is $T$-rigid, it is straightforward to see that (see [45, Section 5])

$$
\begin{aligned}
& \square_{b}^{(q)} Q_{m}^{(q)}=Q_{m}^{(q)} \square_{b}^{(q)} \text { on } \Omega_{0}^{0, q}(X,), \forall m \in \mathbb{Z}, \\
& \bar{\partial}_{b} Q_{m}^{(q)}=Q_{m}^{(q+1)} \bar{\partial}_{b} \text { on } \Omega_{0}^{0, q}(X), \forall m \in \mathbb{Z}, q=0,1, \ldots, n-2, \\
& \bar{\partial}_{b}^{*} Q_{m}^{(q)}=Q_{m}^{(q-1)} \bar{\partial}_{b}^{*} \text { on } \Omega_{0}^{0, q}(X), \forall m \in \mathbb{Z}, q=1, \ldots, n-1 .
\end{aligned}
$$

Now, we assume that $X$ is compact. By using elementary Fourier analysis, it is straightforward to see that for every $u \in \Omega^{0, q}(X)$,

$$
\lim _{N \rightarrow \infty} \sum_{m=-N}^{N} Q_{m}^{(q)} u=u \text { in the } \mathscr{C}^{\infty} \text { topology, }
$$

$$
\sum_{m=-N}^{N}\left\|Q_{m}^{(q)} u\right\|^{2} \leq\|u\|^{2}, \quad \forall N \in \mathbb{N}_{0}
$$

Thus, for every $u \in L_{(0, q)}^{2}(X)$,

$$
\begin{aligned}
& \lim _{N \rightarrow \infty} \sum_{m=-N}^{N} Q_{m}^{(q)} u=u \text { in } L_{(0, q)}^{2}\left(X, L^{k}\right), \\
& \sum_{m=-N}^{N}\left\|Q_{m}^{(q)} u\right\|^{2} \leq\|u\|^{2}, \quad \forall N \in \mathbb{N}_{0} .
\end{aligned}
$$

For $m \in \mathbb{Z}$, put

$$
Q_{\leq m}^{(q)}: L_{(0, q)}^{2}(X) \rightarrow L_{(0, q)}^{2}(X), \quad u \longmapsto \lim _{N \rightarrow \infty} \sum_{j=0}^{N} Q_{m-j}^{(q)} u
$$

and

$$
Q_{\geq m}^{(q)}: L_{(0, q)}^{2}(X) \rightarrow L_{(0, q)}^{2}(X), \quad u \longmapsto \lim _{N \rightarrow \infty} \sum_{j=0}^{N} Q_{m+j}^{(q)} u .
$$

In view of (6.12) and $(6.13)$, we see that $(6.14)$ and $(6.15)$ are well-defined.

The following is straightforward and we omit the proof. 
Theorem 6.6. Let $m \in \mathbb{Z}$, we have

$$
\begin{aligned}
& Q_{\geq m}^{(q)}, Q_{\leq m}^{(q)}: \Omega^{0, q}(X) \rightarrow \Omega^{0, q}(X), \\
& i\left(T Q_{\geq m}^{(q)} u \mid u\right) \geq m\|u\|, \quad \forall u \in \Omega^{0, q}(X), \\
& i\left(T Q_{\leq m}^{(q)} u \mid u\right) \leq m\|u\|, \quad \forall u \in \Omega^{0, q}(X), \\
& Q_{\geq m}^{(q)}, Q_{\leq m}^{(q)}: \operatorname{Dom} \bar{\partial}_{b} \rightarrow \operatorname{Dom} \bar{\partial}_{b}, \\
& Q_{\geq m}^{(q)} \bar{\partial}_{b}=\bar{\partial}_{b} Q_{\geq m}^{(q)} \text { on } \operatorname{Dom} \bar{\partial}_{b}, \\
& Q_{\leq m}^{(q)} \bar{\partial}_{b}=\bar{\partial}_{b} Q_{\leq m}^{(q)} \text { on } \operatorname{Dom} \bar{\partial}_{b}, \\
& Q_{\geq m}^{(q)}, Q_{\leq m}^{(q)}: \operatorname{Dom} \bar{\partial}_{b}^{*} \rightarrow \operatorname{Dom} \bar{\partial}_{b}^{*} \\
& Q_{\geq m}^{(q)} \bar{\partial}_{b}^{*}=\bar{\partial}_{b}^{*} Q_{\geq m}^{(q)} \text { on } \operatorname{Dom} \bar{\partial}_{b}^{*}, \\
& Q_{\leq m}^{(q)} \bar{\partial}_{b}^{*}=\bar{\partial}_{b}^{*} Q_{\leq m}^{(q)} \text { on } \operatorname{Dom} \bar{\partial}_{b}^{*}, \\
& Q_{\geq m}^{(q)}, Q_{\leq m}^{(q)}: \operatorname{Dom}_{b}^{(q)} \rightarrow \operatorname{Dom} \square_{b}^{(q)} \\
& Q_{\geq m}^{(q)} \square_{b}^{(q)}=\square_{b}^{(q)} Q_{\geq m}^{(q)} \text { on } \operatorname{Dom} \square_{b}^{(q)}, \\
& Q_{\leq m}^{(q)} \square_{b}^{(q)}=\square_{b}^{(q)} Q_{\leq m}^{(q)} \text { on } \operatorname{Dom} \square_{b}^{(q)}, \\
& Q_{\geq m}^{(q)} \Pi^{(q)}=\Pi^{(q)} Q_{\geq m}^{(q)} \text { on } L_{(0, q)}^{2}(X), \\
& Q_{\leq m}^{(q)} \Pi^{(q)}=\Pi^{(q)} Q_{\leq m}^{(q)} \text { on } L_{(0, q)}^{2}(X) .
\end{aligned}
$$

To continue, put for $m \in \mathbb{Z}$,

$$
\begin{aligned}
& \mathcal{B}_{\geq m}^{0, q}(X):=\left\{Q_{\geq m}^{(q)} u ; u \in L_{(0, q)}^{2}(X)\right\} \\
& \mathcal{B}_{\leq m}^{0, q}(X):=\left\{Q_{\leq m}^{(q)} u ; u \in L_{(0, q)}^{2}(X)\right\} .
\end{aligned}
$$

Note that $\left(Q_{\leq m}^{(q)}\right)^{2}=Q_{\leq m}^{(q)},\left(Q_{\geq m}^{(q)}\right)^{2}=Q_{\geq m}^{(q)}$. From this observation and (6.16), we see that

$$
\begin{aligned}
& \operatorname{Dom} \square_{b}^{(q)} \cap \mathcal{B}_{\leq m}^{0, q}=\left\{Q_{\leq m}^{(q)} u ; u \in \operatorname{Dom} \square_{b}^{(q)}\right\}, \\
& \operatorname{Dom} \square_{b}^{(q)} \cap \mathcal{B}_{\geq m}^{0, q}=\left\{Q_{\geq m}^{(q)} u ; u \in \operatorname{Dom} \square_{b}^{(q)}\right\},
\end{aligned}
$$

and

$$
\begin{gathered}
\square_{b}^{(q)}: \operatorname{Dom} \square_{b}^{(q)} \cap \mathcal{B}_{\geq m}^{0, q}(X) \rightarrow \mathcal{B}_{\geq m}^{0, q}(X), \\
\square_{b}^{(q)}: \operatorname{Dom} \square_{b}^{(q)} \cap \mathcal{B}_{\leq m}^{0, q}(X) \rightarrow \mathcal{B}_{\leq m}^{0, q}(X) .
\end{gathered}
$$

Thus, it is quite interesting to study the behaviour of $\square_{b}^{(q)}$ in the spaces $\mathcal{B}_{\geq m}^{0, q}$ and $\mathcal{B}_{\leq m}^{0, q}$. We recall now the condition $Z(q)$ of Hörmander.

Definition 6.7. Given $q \in\{0, \ldots, n-1\}$, the Levi form is said to satisfy condition $Z(q)$ at $p \in X$, if $\mathcal{L}_{p}$ has at least $n-q$ positive eigenvalues or at least $q+1$ negative eigenvalues.

Usually, the condition $Z(q)$ is introduced for a smooth domain $D$ with boundary $X=\partial D$ in a complex manifold $M$. Then condition $Z(q)$ implies subelliptic estimates for the $\bar{\partial}$-Neumann problem on $D$, cf. [23, 34]. If one wants to to obtain subelliptic estimates on $X$, one cannot distinguish whether $X$ is the boundary of $D$ or $X$ is the boundary of the complement of $D$. 
Thus, one assumes that condition $Z(q)$ holds on both $D$ and its complement $M \backslash D$. Note that condition $Z(q)$ on $M \backslash D$ is equivalent to condition $Z(n-q-1)$ on $D$. However, we show in the next theorem, that condition $Z(q)$ (resp. $Z(n-q-1)$ ) yields subelliptic estimates on a $C R$ manifold with $S^{1}$ action, by projecting the forms with $Q_{\leq 0}^{(q)}$, (resp. $\left.Q_{\geq 0}^{(q)}\right)$.

Theorem 6.8. With the notations and assumptions above, assume that $Z(q)$ holds at every point of $X$. Then, for every $s \in \mathbb{N}_{0}$, there is a constant $C_{s}>0$ such that

$$
\left\|Q_{\leq 0}^{(q)} u\right\|_{s+1} \leq C_{s}\left(\left\|\square_{b}^{(q)} Q_{\leq 0}^{(q)} u\right\|_{s}+\left\|Q_{\leq 0}^{(q)} u\right\|\right), \quad \forall u \in \Omega^{0, q}(X)
$$

where $\|\cdot\|_{s}$ denotes the usual Sobolev norm of order $s$ on $X$.

Similarly, if $Z(n-1-q)$ holds at every point of $X$, then for every $s \in \mathbb{N}_{0}$, there is a constant $C_{s}>0$ such that

$$
\left\|Q_{\geq 0}^{(q)} u\right\|_{s+1} \leq C_{s}\left(\left\|\square_{b}^{(q)} Q_{\geq 0}^{(q)} u\right\|_{s}+\left\|Q_{\geq 0}^{(q)} u\right\|\right), \quad \forall u \in \Omega^{0, q}(X) .
$$

Proof. If we go through Kohn's $L^{2}$ estimates (see [23, Theorem 8.4.2], [34, Proposition 5.4.10], [50]), we see that:

(I) If $Z(q)$ holds at every point of $X$, then, for every $s \in \mathbb{N}_{0}$, there is a constant $C_{s}>0$ such that for all $u \in \Omega^{0, q}(X)$ with $i(T u \mid u) \leq 0$, we have

$$
\|u\|_{s+1} \leq C_{s}\left(\left\|\square_{b}^{(q)} u\right\|_{s}+\|u\|\right)
$$

(II) If $Z(n-1-q)$ holds at every point of $X$, then, for every $s \in \mathbb{N}_{0}$, there is a constant $\widetilde{C}_{s}>0$ such that for all $u \in \Omega^{0, q}(X)$ with $i(T u \mid u) \geq 0$, we have

$$
\|u\|_{s+1} \leq \widetilde{C}_{s}\left(\left\|\square_{b}^{(q)} u\right\|_{s}+\|u\|\right)
$$

We notice that

$$
i\left(T Q_{\leq 0}^{(q)} u \mid Q_{\leq 0}^{(q)} u\right) \leq 0, \quad i\left(T Q_{\geq 0}^{(q)} u \mid Q_{\geq 0}^{(q)} u\right) \geq 0, \quad \forall u \in \Omega^{0, q}(X) .
$$

From this observation and (I) and (II), the theorem follows.

For every $s \in \mathbb{Z}$, let $H_{-}^{s}\left(X, T^{* 0, q} X\right)$ and $H_{+}^{s}\left(X, T^{* 0, q} X\right)$ denote the completions of $\mathcal{B}_{\leq 0}^{0, q}(X) \cap$ $\Omega^{0, q}(X)$ and $\mathcal{B}_{\geq 0}^{0, q}(X) \cap \Omega^{0, q}(X)$ with respect to $\|\cdot\|_{s}$ respectively. Let $\mathscr{D}_{-}^{\prime}\left(X, T^{* 0, q} X\right)$ and $\mathscr{D}_{+}^{\prime}\left(X, T^{* 0, q} X\right)$ denote the dual spaces of $\mathcal{B}_{\leq 0}^{0, q}(X) \cap \Omega^{0, q}(X)$ and $\mathcal{B}_{\geq 0}^{0, q}(X) \cap \Omega^{0, q}(X)$ respectively.

From Theorem 6.8, we can repeat the method of Kohn (see [23, Chapter 8], [34], [50]) and deduce the following.

Theorem 6.9. With the notations and assumptions above, assume that $Z(q)$ holds at every point of $X$. Then $\square_{b}^{(q)}:$ Dom $\square_{b}^{(q)} \cap \mathcal{B}_{\leq 0}^{0, q}(X) \rightarrow \mathcal{B}_{\leq 0}^{0, q}(X)$ has closed range. Let

$$
N_{-}^{(q)}: \mathcal{B}_{\leq 0}^{0, q}(X) \rightarrow \operatorname{Dom} \square_{b}^{(q)} \cap \mathcal{B}_{\leq 0}^{0, q}(X)
$$

be the associated partial inverse and let

$$
\Pi_{-}^{(q)}: \mathcal{B}_{\leq 0}^{0, q}(X) \rightarrow \operatorname{Ker} \square_{b}^{(q)}
$$


be the orthogonal projection. Then, we have

$$
\begin{aligned}
& \square_{b}^{(q)} N_{-}^{(q)}+\Pi_{-}^{(q)}=I \text { on } \mathcal{B}_{\leq 0}^{0, q}(X), \\
& N_{-}^{(q)} \square_{b}^{(q)}+\Pi_{-}^{(q)}=I \text { on } \mathcal{B}_{\leq 0}^{0, q}(X) \cap \operatorname{Dom} \square_{b}^{(q)}, \\
& N_{-}^{(q)}: H_{-}^{s}\left(X, T^{* 0, q} X\right) \rightarrow H_{-}^{s+1}\left(X, T^{* 0, q} X\right), \forall s \in \mathbb{Z}, \\
& \Pi_{-}^{(q)}: H_{-}^{s}\left(X, T^{* 0, q} X\right) \rightarrow H_{-}^{s+N}\left(X, T^{* 0, q} X\right), \forall s \in \mathbb{Z} \text { and } N \in \mathbb{N} .
\end{aligned}
$$

Moreover, $N_{-}^{(q)}$ and $\Pi_{-}^{(q)}$ can be continuously extended to $\mathcal{D}_{-}^{\prime}\left(X, T^{* 0, q} X\right)$ and we have

$$
\begin{aligned}
& \Pi_{-}^{(q)}: \mathscr{D}_{-}^{\prime}\left(X, T^{* 0, q} X\right) \rightarrow \mathcal{B}_{\leq 0}^{0, q}(X) \bigcap \Omega^{0, q}(X), \\
& N_{-}^{(q)}: \mathscr{D}_{-}^{\prime}\left(X, T^{* 0, q} X\right) \rightarrow \mathscr{D}_{-}^{\prime}\left(X, T^{* 0, q} X\right), \\
& \square_{b}^{(q)} N_{-}^{(q)}+\Pi_{-}^{(q)}=I \text { on } \mathscr{D}_{-}^{\prime}\left(X, T^{* 0, q} X\right), \\
& N_{-}^{(q)} \square_{b}^{(q)}+\Pi_{-}^{(q)}=I \text { on } \mathscr{D}_{-}^{\prime}\left(X, T^{* 0, q} X\right) .
\end{aligned}
$$

Theorem 6.10. With the notations and assumptions above, assume that $Z(n-1-q)$ holds at every point of $X$. Then,

$$
\square_{b}^{(q)}: \operatorname{Dom} \square_{b}^{(q)} \cap \mathcal{B}_{\geq 0}^{0, q}(X) \rightarrow \mathcal{B}_{\geq 0}^{0, q}(X)
$$

has closed range. Let

$$
N_{+}^{(q)}: \mathcal{B}_{\geq 0}^{0, q}(X) \rightarrow \operatorname{Dom} \square_{b}^{(q)} \cap \mathcal{B}_{\geq 0}^{0, q}(X)
$$

be the associated partial inverse and let

$$
\Pi_{+}^{(q)}: \mathcal{B}_{\geq 0}^{0, q}(X) \rightarrow \operatorname{Ker} \square_{b}^{(q)}
$$

be the orthogonal projection. Then, we have

$$
\begin{aligned}
& \square_{b}^{(q)} N_{+}^{(q)}+\Pi_{+}^{(q)}=I \text { on } \mathcal{B}_{\geq 0}^{0, q}(X), \\
& N_{+}^{(q)} \square_{b}^{(q)}+\Pi_{+}^{(q)}=I \text { on } \mathcal{B}_{\geq 0}^{0, q}(X) \cap \operatorname{Dom} \square_{b}^{(q)}, \\
& N_{+}^{(q)}: H_{+}^{s}\left(X, T^{* 0, q} X\right) \rightarrow H_{+}^{s+1}\left(X, T^{* 0, q} X\right), \forall s \in \mathbb{Z}, \\
& \Pi_{+}^{(q)}: H_{+}^{s}\left(X, T^{* 0, q} X\right) \rightarrow H_{+}^{s+N}\left(X, T^{* 0, q} X\right), \forall s \in \mathbb{Z} \text { and } N \in \mathbb{N} .
\end{aligned}
$$

Moreover, $N_{+}^{(q)}$ and $\Pi_{+}^{(q)}$ can be continuously extended to $\mathcal{D}_{+}^{\prime}\left(X, T^{* 0, q} X\right)$ and we have

$$
\begin{aligned}
& \Pi_{+}^{(q)}: \mathscr{D}_{+}^{\prime}\left(X, T^{* 0, q} X\right) \rightarrow \mathcal{B}_{\geq 0}^{0, q}(X) \bigcap \Omega^{0, q}(X), \\
& N_{+}^{(q)}: \mathscr{D}_{+}^{\prime}\left(X, T^{* 0, q} X\right) \rightarrow \mathscr{D}_{+}^{\prime}\left(X, T^{* 0, q} X\right), \\
& \square_{b}^{(q)} N_{+}^{(q)}+\Pi_{+}^{(q)}=I \text { on } \mathscr{D}_{+}^{\prime}\left(X, T^{* 0, q} X\right), \\
& N_{+}^{(q)} \square_{b}^{(q)}+\Pi_{+}^{(q)}=I \text { on } \mathscr{D}_{+}^{\prime}\left(X, T^{* 0, q} X\right) .
\end{aligned}
$$

Our next goal is to prove that if $Z(q)$ fails but $Z(q-1)$ and $Z(q+1)$ hold at every point of $X$, then,

$$
\square_{b}^{(q)}: \operatorname{Dom} \square_{b}^{(q)} \cap \mathcal{B}_{\leq 0}^{0, q}(X) \rightarrow \mathcal{B}_{\leq 0}^{0, q}(X)
$$

has closed range. Until further notice, we assume that $Z(q)$ fails but $Z(q-1)$ and $Z(q+1)$ hold at every point of $X$. Let $N_{-}^{(q-1)}$ and $N_{-}^{(q+1)}$ be as in Theorem 6.9. We first need the following. 
Lemma 6.11. Let $u \in \mathcal{B}_{\leq 0}^{0, q}(X)$. We have

$$
\bar{\partial}_{b}^{*} \bar{\partial}_{b} N_{-}^{(q+1)} \bar{\partial}_{b} u=0
$$

and

$$
\bar{\partial}_{b} \bar{\partial}_{b}^{*} N_{-}^{(q-1)} \bar{\partial}_{b}^{*} u=0
$$

Proof. Let $u \in \mathcal{B}_{\leq 0}^{0, q}(X)$. Take $u_{j} \in \mathcal{B}_{\leq 0}^{0, q}(X) \cap \Omega^{0, q}(X), j=1,2, \ldots$, so that $u_{j} \rightarrow u$ in $L_{(0, q)}^{2}(X)$ as $j \rightarrow \infty$. Then, $\bar{\partial}_{b}^{*} \bar{\partial}_{b} N_{-}^{(q+1)} \bar{\partial}_{b} u_{j} \rightarrow \bar{\partial}_{b}^{*} \bar{\partial}_{b} N_{-}^{(q+1)} \bar{\partial}_{b} u$ in $\mathscr{D}_{-}^{\prime}\left(X, T^{* 0, q} X\right)$ as $j \rightarrow \infty$. Fix $j=1,2, \ldots$ From (6.21), we have

$$
\begin{aligned}
\bar{\partial}_{b}^{*} \bar{\partial}_{b} N_{-}^{(q+1)} \bar{\partial}_{b} u_{j} & =N_{-}^{(q+1)} \square_{b}^{(q+1)} \bar{\partial}_{b}^{*} \bar{\partial}_{b} N_{-}^{(q+1)} \bar{\partial}_{b} u_{j} \\
& =N_{-}^{(q+1)} \bar{\partial}_{b}^{*} \bar{\partial}_{b} \square_{b}^{(q+1)} N_{-}^{(q+1)} \bar{\partial}_{b} u_{j} \\
& =N_{-}^{(q+1)} \bar{\partial}_{b}^{*} \bar{\partial}_{b}\left(I-\Pi_{-}^{(q+1)}\right) \bar{\partial}_{b} u_{j} \\
& =N_{-}^{(q+1)} \bar{\partial}_{b}^{*} \bar{\partial}_{b}^{2} u_{j}=0 .
\end{aligned}
$$

Hence $\bar{\partial}_{b}^{*} \bar{\partial}_{b} N_{-}^{(q+1)} \bar{\partial}_{b} u=0$. (6.25) follows. The proof of (6.26) is essentially the same.

Lemma 6.12. The following operators are continuous:

$$
\begin{aligned}
& \bar{\partial}_{b} N_{-}^{(q-1)} \bar{\partial}_{b}^{*}: \mathcal{B}_{\leq 0}^{0, q}(X) \rightarrow \mathcal{B}_{\leq 0}^{0, q}(X), \\
& \bar{\partial}_{b}^{*} N_{-}^{(q+1)} \bar{\partial}_{b}: \mathcal{B}_{\leq 0}^{0, q}(X) \rightarrow \mathcal{B}_{\leq 0}^{0, q}(X) .
\end{aligned}
$$

Moreover, for every $u \in \mathcal{B}_{\leq 0}^{0, q}(X)$,

$$
u-\left(\bar{\partial}_{b} N_{-}^{(q-1)} \bar{\partial}_{b}^{*}+\bar{\partial}_{b}^{*} N_{-}^{(q+1)} \bar{\partial}_{b}\right) u \in \operatorname{ker} \square_{b}^{(q)} \cap \mathcal{B}_{\leq 0}^{0, q}(X) .
$$

Proof. Let $u \in \mathcal{B}_{\leq 0}^{(0, q)} \cap \Omega^{0, q}(X)$. We have

$$
\begin{aligned}
& \left\|\bar{\partial}_{b}^{*} N_{-}^{(q+1)} \bar{\partial}_{b} u\right\|^{2} \\
& =\left(\bar{\partial}_{b}^{*} N_{-}^{(q+1)} \bar{\partial}_{b} u \mid \bar{\partial}_{b}^{*} N_{-}^{(q+1)} \bar{\partial}_{b} u\right)=\left(\bar{\partial}_{b} \bar{\partial}_{b}^{*} N_{-}^{(q+1)} \bar{\partial}_{b} u \mid N_{-}^{(q+1)} \bar{\partial}_{b} u\right) \\
& \left.=\left(\square_{b}^{(q+1)} N_{-}^{(q+1)} \bar{\partial}_{b} u \mid N_{-}^{(q+1)} \bar{\partial}_{b} u\right) \text { (here we used (6.25) }\right) \\
& =\left(\bar{\partial}_{b} u \mid N_{-}^{(q+1)} \bar{\partial}_{b} u\right)=\left(u \mid \bar{\partial}_{b}^{*} N_{-}^{(q+1)} \bar{\partial}_{b} u\right) \\
& \leq\|u\|\left\|\bar{\partial}_{b}^{*} N_{-}^{(q+1)} \bar{\partial}_{b} u\right\| .
\end{aligned}
$$

Hence, $\left\|\bar{\partial}_{b}^{*} N_{-}^{(q+1)} \bar{\partial}_{b} u\right\| \leq\|u\|, \forall u \in \mathcal{B}_{\leq 0}^{0, q}(X) \cap \Omega^{0, q}(X)$. Thus, $\bar{\partial}_{b}^{*} N_{-}^{(q+1)} \bar{\partial}_{b}$ can be continuously extended to $\bar{\partial}_{b}^{*} N_{-}^{(q+1)} \bar{\partial}_{b}: \mathcal{B}_{\leq 0}^{0, q}(X) \rightarrow \mathcal{B}_{\leq 0}^{0, q}(X)$.

Similarly, we can repeat the procedure above and conclude that

$$
\bar{\partial}_{b} N_{-}^{(q-1)} \bar{\partial}_{b}^{*}: \mathcal{B}_{\leq 0}^{0, q}(X) \rightarrow \mathcal{B}_{\leq 0}^{0, q}(X) \text { is continuous. }
$$

(6.27) follows.

Let $u \in \mathcal{B}_{\leq 0}^{0, q}(X) \cap \Omega^{0, q}(X)$ and set $v=u-\left(\bar{\partial}_{b} N_{-}^{(q-1)} \bar{\partial}_{b}^{*}+\bar{\partial}_{b}^{*} N_{-}^{(q+1)} \bar{\partial}_{b}\right) u$. We have

$$
\begin{aligned}
\bar{\partial}_{b} v & =\bar{\partial}_{b} u-\bar{\partial}_{b} \bar{\partial}_{b}^{*} N_{-}^{(q+1)} \bar{\partial}_{b} u \\
& \left.=\bar{\partial}_{b} u-\square_{b}^{(q+1)} N_{-}^{(q+1)} \bar{\partial}_{b} u \quad \text { (here we used (6.25) }\right) \\
& =\bar{\partial}_{b} u-\bar{\partial}_{b} u=0
\end{aligned}
$$


Similarly, we have $\bar{\partial}_{b}^{*} v=0$. Thus,

$$
u-\left(\bar{\partial}_{b} N_{-}^{(q-1)} \bar{\partial}_{b}^{*}+\bar{\partial}_{b}^{*} N_{-}^{(q+1)} \bar{\partial}_{b}\right) u \in \operatorname{Ker} \square_{b}^{(q)},
$$

for every $u \in \mathcal{B}_{\leq 0}^{0, q}(X) \cap \Omega^{0, q}(X)$. Since

$$
I-\bar{\partial}_{b} N_{-}^{(q-1)} \bar{\partial}_{b}^{*}-\bar{\partial}_{b}^{*} N_{-}^{(q+1)} \bar{\partial}_{b}: \mathcal{B}_{\leq 0}^{0, q}(X) \rightarrow \mathcal{B}_{\leq 0}^{0, q}(X)
$$

is continuous, 6.28) follows.

Let $\bar{\partial}_{b}^{*, f}: \Omega^{0, q+1}(X) \rightarrow \Omega^{0, q}(X)$ be the formal adjonit of $\bar{\partial}_{b}$ with respect to $(\cdot \mid \cdot)$. That is, $\left(\bar{\partial}_{b} f \mid g\right)=\left(f \mid \bar{\partial}_{b}^{*, f} g\right)$, for all $f \in \Omega^{0, q}(X), g \in \Omega^{0, q+1}(X)$. We need

Lemma 6.13. Let $u \in L_{(0, q)}^{2}(X)$. If $\bar{\partial}_{b}^{*, f} u \in L_{(0, q-1)}^{2}(X)$, then $u \in \operatorname{Dom} \bar{\partial}_{b}^{*}$ and $\bar{\partial}_{b}^{*} u=\bar{\partial}_{b}^{*, f} u$.

Proof. Let $g \in \operatorname{Dom} \bar{\partial}_{b} \subset L_{(0, q-1)}^{2}(X)$. From Friedrichs' lemma [23, Corollary D.2], we can find $g_{j} \in \Omega^{0, q-1}(X), j=1,2, \ldots$, such that $g_{j} \rightarrow g$ in $L_{(0, q-1)}^{2}(X)$ as $j \rightarrow \infty$ and $\bar{\partial}_{b} g_{j} \rightarrow \bar{\partial}_{b} g$ in $L_{(0, q)}^{2}(X)$ as $j \rightarrow \infty$. We have

$$
\left(u \mid \bar{\partial}_{b} g\right)=\lim _{j \rightarrow \infty}\left(u \mid \bar{\partial}_{b} g_{j}\right)=\lim _{j \rightarrow \infty}\left(\bar{\partial}_{b}^{*, f} u \mid g_{j}\right)=\left(\bar{\partial}_{b}^{*, f} u \mid g\right) .
$$

Thus, $u \in \operatorname{Dom} \bar{\partial}_{b}^{*}$ and $\bar{\partial}_{b}^{*} u=\bar{\partial}_{b}^{*, f} u$. The lemma follows.

Lemma 6.14. We have

$$
\bar{\partial}_{b}^{*}\left(N_{-}^{(q+1)}\right)^{2} \bar{\partial}_{b}: \mathcal{B}_{\leq 0}^{0, q}(X) \rightarrow \operatorname{Dom} \square_{b}^{(q)} \cap \mathcal{B}_{\leq 0}^{0, q}(X)
$$

and

$$
\bar{\partial}_{b}\left(N_{-}^{(q-1)}\right)^{2} \bar{\partial}_{b}^{*}: \mathcal{B}_{\leq 0}^{0, q}(X) \rightarrow \operatorname{Dom} \square_{b}^{(q)} \cap \mathcal{B}_{\leq 0}^{0, q}(X) .
$$

Proof. In view of (6.21), we see that

$$
\bar{\partial}_{b}^{*}\left(N_{-}^{(q+1)}\right)^{2} \bar{\partial}_{b}: \mathcal{B}_{\leq 0}^{0, q}(X) \rightarrow \mathcal{B}_{\leq 0}^{0, q}(X) \text { is continuous. }
$$

Let $u \in \mathcal{B}_{\leq 0}^{0, q}(X) \cap \Omega^{0, q}(X)$. We have

$$
\begin{aligned}
\left\|\bar{\partial}_{b} \bar{\partial}_{b}^{*}\left(N_{-}^{(q+1)}\right)^{2} \bar{\partial}_{b} u\right\|^{2} & =\left(\bar{\partial}_{b} \bar{\partial}_{b}^{*}\left(N_{-}^{(q+1)}\right)^{2} \bar{\partial}_{b} u \mid \bar{\partial}_{b} \bar{\partial}_{b}^{*}\left(N_{-}^{(q+1)}\right)^{2} \bar{\partial}_{b} u\right) \\
& =\left(\bar{\partial}_{b}^{*} \bar{\partial}_{b} \bar{\partial}_{b}^{*}\left(N_{-}^{(q+1)}\right)^{2} \bar{\partial}_{b} u \mid \bar{\partial}_{b}^{*}\left(N_{-}^{(q+1)}\right)^{2} \bar{\partial}_{b} u\right) \\
& =\left(\bar{\partial}_{b}^{*} \square_{b}^{(q+1)}\left(N_{-}^{(q+1)}\right)^{2} \bar{\partial}_{b} u \mid \bar{\partial}_{b}^{*}\left(N_{-}^{(q+1)}\right)^{2} \bar{\partial}_{b} u\right) \\
& =\left(\bar{\partial}_{b}^{*} N_{-}^{(q+1)} \bar{\partial}_{b} u \mid \bar{\partial}_{b}^{*}\left(N_{-}^{(q+1)}\right)^{2} \bar{\partial}_{b} u\right) \\
& \leq\left\|\bar{\partial}_{b}^{*} N_{-}^{(q+1)} \bar{\partial}_{b} u\right\|\left\|\bar{\partial}_{b}^{*}\left(N_{-}^{(q+1)}\right)^{2} \bar{\partial}_{b} u\right\| .
\end{aligned}
$$

From (6.27) and (6.31), we see that there is a constant $C>0$ such that

$$
\left\|\bar{\partial}_{b} \bar{\partial}_{b}^{*}\left(N_{-}^{(q+1)}\right)^{2} \bar{\partial}_{b} u\right\| \leq C\|u\|, \quad \forall u \in \mathcal{B}_{\leq 0}^{0, q}(X) \cap \Omega^{0, q}(X) .
$$

Thus, $\bar{\partial}_{b} \bar{\partial}_{b}^{*}\left(N_{-}^{(q+1)}\right)^{2} \bar{\partial}_{b}$ can be extended continuously to $\mathcal{B}_{\leq 0}^{0, q}(X)$ and we have

$$
\bar{\partial}_{b} \bar{\partial}_{b}^{*}\left(N_{-}^{(q+1)}\right)^{2} \bar{\partial}_{b}: \mathcal{B}_{\leq 0}^{0, q}(X) \rightarrow \mathcal{B}_{\leq 0}^{0, q+1}(X) \text { is continuous. }
$$

Hence,

$$
\bar{\partial}_{b}^{*}\left(N_{-}^{(q+1)}\right)^{2} \bar{\partial}_{b}: \mathcal{B}_{\leq 0}^{0, q}(X) \rightarrow \operatorname{Dom} \bar{\partial}_{b} \cap \mathcal{B}_{\leq 0}^{0, q}(X)
$$


Let $u \in \mathcal{B}_{\leq 0}^{0, q}(X) \cap \Omega^{0, q}(X)$. We have

$$
\begin{aligned}
\bar{\partial}_{b}^{*, f} \bar{\partial}_{b} \bar{\partial}_{b}^{*}\left(N_{-}^{(q+1)}\right)^{2} \bar{\partial}_{b} u & =\bar{\partial}_{b}^{*} \bar{\partial}_{b} \bar{\partial}_{b}^{*}\left(N_{-}^{(q+1)}\right)^{2} \bar{\partial}_{b} u \\
& =\bar{\partial}_{b}^{*} \square_{b}^{(q+1)}\left(N_{-}^{(q+1)}\right)^{2} \bar{\partial}_{b} u \\
& =\bar{\partial}_{b}^{*} N_{-}^{(q+1)} \bar{\partial}_{b} u .
\end{aligned}
$$

From (6.27) and (6.33), we see that there is a constant $C_{1}>0$ such that

$$
\left\|\bar{\partial}_{b}^{*, f} \bar{\partial}_{b} \bar{\partial}_{b}^{*}\left(N_{-}^{(q+1)}\right)^{2} \bar{\partial}_{b} u\right\| \leq C_{1}\|u\|, \quad \forall u \in \mathcal{B}_{\leq 0}^{0, q}(X) \cap \Omega^{0, q}(X) .
$$

Thus, $\bar{\partial}_{b}^{*, f} \bar{\partial}_{b} \bar{\partial}_{b}^{*}\left(N_{-}^{(q+1)}\right)^{2} \bar{\partial}_{b}$ can be extended continuously to $\mathcal{B}_{\leq 0}^{0, q}(X)$ and we have

$$
\bar{\partial}_{b}^{*, f} \bar{\partial}_{b} \bar{\partial}_{b}^{*}\left(N_{-}^{(q+1)}\right)^{2} \bar{\partial}_{b}: \mathcal{B}_{\leq 0}^{0, q}(X) \rightarrow \mathcal{B}_{\leq 0}^{0, q}(X) \text { is continuous. }
$$

From (6.34) and Lemma 6.13, we conclude that

$$
\bar{\partial}_{b} \bar{\partial}_{b}^{*}\left(N_{-}^{(q+1)}\right)^{2} \bar{\partial}_{b}: \mathcal{B}_{\leq 0}^{0, q}(X) \rightarrow \operatorname{Dom} \bar{\partial}_{b}^{*} \cap \mathcal{B}_{\leq 0}^{0, q+1}(X)
$$

Moreover, it is easy to see that for $u \in \mathcal{B}_{\leq 0}^{0, q}(X), \bar{\partial}_{b}^{*}\left(N_{-}^{(q+1)}\right)^{2} \bar{\partial}_{b} u \in \operatorname{Dom} \bar{\partial}_{b}^{*}$ and

$$
\left(\bar{\partial}_{b}^{*}\right)^{2}\left(N_{-}^{(q+1)}\right)^{2} \bar{\partial}_{b} u=0 .
$$

From this observation, (6.35) and (6.32), (6.29) follows.

The proof of (6.30) is essentially the same.

Theorem 6.15. With the notations above, assume that $Z(q)$ fails but $Z(q-1)$ and $Z(q+1)$ hold at every point of $X$. Then,

$$
\square_{b}^{(q)}: \operatorname{Dom} \square_{b}^{(q)} \cap \mathcal{B}_{\leq 0}^{0, q}(X) \rightarrow \mathcal{B}_{\leq 0}^{0, q}(X)
$$

has closed range.

Proof. Let $\square_{b}^{(q)} u_{j}=v_{j}, u_{j} \in \operatorname{Dom}_{b}^{(q)} \cap \mathcal{B}_{\leq 0}^{0, q}(X), v_{j} \in \mathcal{B}_{\leq 0}^{0, q}(X), j=1,2, \ldots$, with $v_{j} \rightarrow v \in$ $\mathcal{B}_{\leq 0}^{0, q}(X)$ as $j \rightarrow \infty$. We are going to prove that there is a $g \in \operatorname{Dom} \square_{b}^{(q)} \cap \mathcal{B}_{\leq 0}^{0, q}(X)$ such that $\square_{b}^{(q)} g=v$. Let $N_{-}^{(q-1)}$ and $N_{-}^{(q+1)}$ be as in Theorem 6.9. Put

$$
g_{j}=\left(\bar{\partial}_{b}^{*} N_{-}^{(q+1)} \bar{\partial}_{b}+\bar{\partial}_{b} N_{-}^{(q-1)} \bar{\partial}_{b}^{*}\right) u_{j}, \quad j=1,2, \ldots
$$

In view of (6.27), we see that $g_{j} \in \mathcal{B}_{\leq 0}^{0, q}(X)$. Moreover, from (6.28), we have

$$
u_{j}-\left(\bar{\partial}_{b}^{*} N_{-}^{(q+1)} \bar{\partial}_{b}+\bar{\partial}_{b} N_{-}^{(q-1)} \bar{\partial}_{b}^{*}\right) u_{j} \in \operatorname{Ker} \square_{b}^{(q)} \subset \operatorname{Dom}_{b}^{(q)}, \quad j=1,2, \ldots .
$$

Hence,

$$
\begin{aligned}
& g_{j} \in \operatorname{Dom} \square_{b}^{(q)} \cap \mathcal{B}_{\leq 0}^{0, q}(X), \quad j=1,2, \ldots, \\
& \square_{b}^{(q)} g_{j}=\square_{b}^{(q)} u_{j}=v_{j}, \quad j=1,2, \ldots
\end{aligned}
$$

We claim that for each $j$,

$$
\begin{aligned}
& g_{j}=\left(\bar{\partial}_{b}^{*} N_{-}^{(q+1)} \bar{\partial}_{b}+\bar{\partial}_{b} N_{-}^{(q-1)} \bar{\partial}_{b}^{*}\right) u_{j} \\
& =\left(\bar{\partial}_{b}^{*}\left(N_{-}^{(q+1)}\right)^{2} \bar{\partial}_{b}+\bar{\partial}_{b}\left(N_{-}^{(q-1)}\right)^{2} \bar{\partial}_{b}^{*}\right) \square_{b}^{(q)} u_{j} \\
& =\left(\bar{\partial}_{b}^{*}\left(N_{-}^{(q+1)}\right)^{2} \bar{\partial}_{b}+\bar{\partial}_{b}\left(N_{-}^{(q-1)}\right)^{2} \bar{\partial}_{b}^{*}\right) v_{j} .
\end{aligned}
$$


Fix $j=1,2, \ldots$. Let $f_{s} \in \mathcal{B}_{\leq 0}^{0, q}(X) \cap \Omega^{0, q}(X), s=1,2, \ldots$, with $f_{s} \rightarrow u_{j}$ in $\mathcal{B}_{\leq 0}^{0, q}(X)$ as $s \rightarrow \infty$. We have

$$
\begin{aligned}
& \left(\bar{\partial}_{b}^{*}\left(N_{-}^{(q+1)}\right)^{2} \bar{\partial}_{b}+\bar{\partial}_{b}\left(N_{-}^{(q-1)}\right)^{2} \bar{\partial}_{b}^{*}\right) \square_{b}^{(q)} f_{s} \\
& \rightarrow\left(\bar{\partial}_{b}^{*}\left(N_{-}^{(q+1)}\right)^{2} \bar{\partial}_{b}+\bar{\partial}_{b}\left(N_{-}^{(q-1)}\right)^{2} \bar{\partial}_{b}^{*}\right) \square_{b}^{(q)} u_{j} \text { in } \mathscr{D}_{-}^{\prime}\left(X, T^{* 0, q} X\right) \text { as } s \rightarrow \infty .
\end{aligned}
$$

We can check that

$$
\begin{aligned}
& \left(\bar{\partial}_{b}^{*}\left(N_{-}^{(q+1)}\right)^{2} \bar{\partial}_{b}+\bar{\partial}_{b}\left(N_{-}^{(q-1)}\right)^{2} \bar{\partial}_{b}^{*}\right) \square_{b}^{(q)} f_{s} \\
& =\bar{\partial}_{b}^{*}\left(N_{-}^{(q+1)}\right)^{2} \square_{b}^{(q+1)} \bar{\partial}_{b} f_{s}+\bar{\partial}_{b}\left(N_{-}^{(q-1)}\right)^{2} \square_{b}^{(q-1)} \bar{\partial}_{b}^{*} f_{s} \\
& =\bar{\partial}_{b}^{*} N_{-}^{(q+1)}\left(I-\Pi_{-}^{(q+1)}\right) \bar{\partial}_{b} f_{s}+\bar{\partial}_{b} N_{-}^{(q-1)}\left(I-\Pi_{-}^{(q-1)}\right) \bar{\partial}_{b}^{*} f_{s} \\
& =\bar{\partial}_{b}^{*} N_{-}^{(q+1)} \bar{\partial}_{b} f_{s}+\bar{\partial}_{b} N_{-}^{(q-1)} \bar{\partial}_{b}^{*} f_{s} \\
& \rightarrow\left(\bar{\partial}_{b}^{*} N_{-}^{(q+1)} \bar{\partial}_{b}+\bar{\partial}_{b} N_{-}^{(q-1)} \bar{\partial}_{b}^{*}\right) u_{j}=g_{j} \text { in } \mathscr{D}_{-}^{\prime}\left(X, T^{* 0, q} X\right) \text { as } s \rightarrow \infty .
\end{aligned}
$$

From (6.38) and (6.39), (6.37) follows. Since $v_{j} \rightarrow v \in \mathcal{B}_{\leq 0}^{0, q}(X)$ and

$$
\bar{\partial}_{b}^{*}\left(N_{-}^{(q+1)}\right)^{2} \bar{\partial}_{b}+\bar{\partial}_{b}\left(N_{-}^{(q-1)}\right)^{2} \bar{\partial}_{b}^{*}: \mathcal{B}_{\leq 0}^{0, q}(X) \rightarrow \mathcal{B}_{\leq 0}^{0, q}(X) \text { is continuous }
$$

(see (6.21) ), we conclude that

$$
g_{j} \rightarrow g:=\left(\bar{\partial}_{b}^{*}\left(N_{-}^{(q+1)}\right)^{2} \bar{\partial}_{b}+\bar{\partial}_{b}\left(N_{-}^{(q-1)}\right)^{2} \bar{\partial}_{b}^{*}\right) v \in \mathcal{B}_{\leq 0}^{0, q}(X)
$$

and $\square_{b}^{(q)} g=v$ in $\mathscr{D}_{-}^{\prime}\left(X, T^{* 0, q} X\right)$. In view of Lemma 6.14, we see that $g \in \operatorname{Dom} \square_{b}^{(q)} \cap \mathcal{B}_{\leq 0}^{0, q}(X)$. The theorem follows.

We can repeat the proof of Theorem 6.15 and deduce the following.

Theorem 6.16. With the notations above, assume that $Z(n-1-q)$ fails but $Z(n-2-q)$ and $Z(n-q)$ hold at every point of $X$. Then,

$$
\square_{b}^{(q)}: \operatorname{Dom} \square_{b}^{(q)} \cap \mathcal{B}_{\geq 0}^{0, q}(X) \rightarrow \mathcal{B}_{\geq 0}^{0, q}(X)
$$

has closed range.

Now, we can prove:

Theorem 6.17. With the notations above, assume that $Z(q)$ fails but $Z(q-1)$ and $Z(q+1)$ hold at every point of $X$. Then, $\square_{b}^{(q)}$ has local $L^{2}$ closed range on $X$ with respect to $Q_{\leq 0}^{(q)}$ in the sense of Definition 1.8

Proof. From Theorem 6.15, we see that there is a constant $C>0$ such that

$$
\left\|\left(I-\Pi_{-}^{(q)}\right) u\right\| \leq C\left\|\square_{b}^{(q)} u\right\|, \quad \forall u \in \mathcal{B}_{\leq 0}^{0, q} \cap \operatorname{Dom} \square_{b}^{(q)} .
$$

Let $f \in \Omega^{0, q}(X)$. Then, $Q_{\leq 0}^{(q)} f \in \mathcal{B}_{\leq 0}^{0, q} \cap \Omega^{0, q}(X)$. We claim that

$$
Q_{\leq 0}^{(q)} \Pi^{(q)} f=\Pi_{-}^{(q)} Q_{\leq 0}^{(q)} f .
$$

Note that $Q_{\leq 0}^{(q)} \Pi^{(q)} f=\Pi^{(q)} Q_{\leq 0}^{(q)} f$. Thus,

$$
\left(Q_{\leq 0}^{(q)} \Pi^{(q)} f \mid Q_{\leq 0}^{(q)}\left(I-\Pi^{(q)}\right) f\right)=\left(\Pi^{(q)} Q_{\leq 0}^{(q)} f \mid\left(I-\Pi^{(q)}\right) Q_{\leq 0}^{(q)} f\right)=0 .
$$


We have the orthogonal decompositions

$$
\begin{aligned}
& Q_{\leq 0}^{(q)} f=Q_{\leq 0}^{(q)} \Pi^{(q)} f+Q_{\leq 0}^{(q)}\left(I-\Pi^{(q)}\right) f, \\
& Q_{\leq 0}^{(q)} f=\Pi_{-}^{(q)} Q_{\leq 0}^{(q)} f+\left(I-\Pi_{-}^{(q)}\right) Q_{\leq 0}^{(q)} f .
\end{aligned}
$$

Hence,

$$
Q_{\leq 0}^{(q)} \Pi^{(q)} f-\Pi_{-}^{(q)} Q_{\leq 0}^{(q)} f=\left(I-\Pi_{-}^{(q)}\right) Q_{\leq 0}^{(q)} f-Q_{\leq 0}^{(q)}\left(I-\Pi^{(q)}\right) f .
$$

From (6.43), we have

$$
\begin{aligned}
& \left(Q_{\leq 0}^{(q)} \Pi^{(q)} f-\Pi_{-}^{(q)} Q_{\leq 0}^{(q)} f \mid Q_{\leq 0}^{(q)} \Pi^{(q)} f-\Pi_{-}^{(q)} Q_{\leq 0}^{(q)} f\right) \\
& =\left(Q_{\leq 0}^{(q)} \Pi^{(q)} f-\Pi_{-}^{(q)} Q_{\leq 0}^{(q)} f \mid\left(I-\Pi_{-}^{(q)}\right) Q_{\leq 0}^{(q)} f-Q_{\leq 0}^{(q)}\left(I-\Pi^{(q)}\right) f\right) \\
& =0
\end{aligned}
$$

since $Q_{\leq 0}^{(q)} \Pi^{(q)} f-\Pi_{-}^{(q)} Q_{\leq 0}^{(q)} f \in \operatorname{Ker} \square_{b}^{(q)} \cap \mathcal{B}_{\leq 0}^{0, q}$. Hence,

$$
Q_{\leq 0}^{(q)} \Pi^{(q)} f=\Pi_{-}^{(q)} Q_{\leq 0}^{(q)} f .
$$

The claim (6.41) follows. Note that $Q_{\leq 0}^{(q)}: \operatorname{Dom}_{b}^{(q)} \cap L_{(0, q)}^{2}(X) \rightarrow \operatorname{Dom} \square_{b}^{(q)} \cap \mathcal{B}_{\leq 0}^{0, q}(X)$ and $Q_{\leq 0}^{(q)}: \Omega^{0, q}(X) \rightarrow \mathcal{B}_{\leq 0}^{0, q}(X) \cap \Omega^{0, q}(X)$. From this observation, (6.41) and (6.40), we obtain

$$
\begin{aligned}
\left\|Q_{\leq 0}^{(q)}\left(I-\Pi^{(q)}\right) u\right\| & =\left\|\left(I-\Pi_{-}^{(q)}\right) Q_{\leq 0}^{(q)} u\right\| \leq C\left\|\square_{b}^{(q)} Q_{\leq 0}^{(q)} u\right\|=C\left\|Q_{\leq 0}^{(q)} \square_{b}^{(q)} u\right\| \\
& \leq C\left\|\square_{b}^{(q)} u\right\|, \quad \forall u \in \Omega^{0, q}(X),
\end{aligned}
$$

where $C>0$ is a constant. The theorem follows.

Similarly, we can repeat the proof of Theorem 6.17 and deduce

Theorem 6.18. With the notations above, assume that $Z(n-1-q)$ fails but $Z(n-2-q)$ and $Z(n-q)$ hold at every point of $X$. Then, $\square_{b}^{(q)}$ has local $L^{2}$ closed range on $X$ with respect to $Q_{\geq 0}^{(q)}$ in the sense of Definition 1.8

Let $D \subset X$ be a canonical coordinate patch and let $x=\left(x_{1}, \ldots, x_{2 n-1}\right)$ be canonical coordinates on $D$ as in Theorem 6.5. We identify $D$ with $W \times]-\pi, \pi\left[\subset \mathbb{R}^{2 n-1}\right.$, where $W$ is some open set in $\mathbb{R}^{2 n-2}$. Until further notice, we work with canonical coordinates $x=\left(x_{1}, \ldots, x_{2 n-1}\right)$. Let $\eta=\left(\eta_{1}, \ldots, \eta_{2 n-1}\right)$ be the dual coordinates of $x$. Let $\alpha\left(x_{2 n-1}\right) \in \mathscr{C}^{\infty}(\mathbb{R},[0,1])$ with $\alpha=1$ on $\left[\frac{1}{2}, \infty[, \alpha=0\right.$ on $\left.]-\infty, \frac{1}{4}\right]$. We recall Definition 2.4 . We need

Lemma 6.19. With the notations above,

$$
\begin{gathered}
Q_{\leq 0}^{(q)}, Q_{\geq 0}^{(q)} \in L_{\mathrm{cl}}^{0}\left(X, T^{* 0, q} X \otimes T^{* 0, q} X\right), \\
Q_{\leq 0}^{(q)}(x, y) \equiv \frac{1}{(2 \pi)^{2 n-1}} \int e^{i\langle x-y, \eta\rangle} \alpha\left(\eta_{2 n-1}\right) d \eta_{2 n-1} \text { at } \Sigma^{-} \cap T^{*} D, \\
Q_{\leq 0}^{(q)}(x, y) \equiv 0 \text { at } \Sigma^{+} \cap T^{*} D
\end{gathered}
$$

and

$$
\begin{aligned}
& Q_{\geq 0}^{(q)}(x, y) \equiv \frac{1}{(2 \pi)^{2 n-1}} \int e^{i\langle x-y, \eta\rangle} \alpha\left(-\eta_{2 n-1}\right) d \eta_{2 n-1} \text { at } \Sigma^{+} \cap T^{*} D \\
& Q_{\geq 0}^{(q)}(x, y) \equiv 0 \text { at } \Sigma^{-} \cap T^{*} D
\end{aligned}
$$


Proof. It is easy to see that on $D$,

$$
Q_{\leq 0}^{(q)} u(y)=\frac{1}{2 \pi} \sum_{m \in \mathbb{Z}, m \geq 0} e^{i m y_{2 n-1}} \int_{-\pi}^{\pi} e^{-i m t} u\left(y^{\prime}, t\right) d t, \quad \forall u \in \Omega_{0}^{0, q}(D)
$$

where $y^{\prime}=\left(y_{1}, \ldots, y_{2 n-2}\right)$. Fix $D^{\prime} \Subset D$ and let $\chi\left(y_{2 n-1}\right) \in \mathscr{C}_{0}^{\infty}(]-\pi, \pi[)$ such that $\chi\left(y_{2 n-1}\right)=1$ for every $\left(y^{\prime}, y_{2 n-1}\right) \in D^{\prime}$. Let $\beta\left(x_{2 n-1}\right) \in \mathscr{C}^{\infty}(\mathbb{R},[0,1])$ with $\beta=1$ on $\left[-\frac{1}{4}, \infty[, \beta=0\right.$ on ] $-\infty,-\frac{1}{2}$ ]. Let $R: \Omega_{0}^{0, q}\left(D^{\prime}\right) \rightarrow \Omega^{0, q}\left(D^{\prime}\right)$ be the continuous operator given by

$$
\begin{array}{r}
u \mapsto \frac{1}{(2 \pi)^{2}} \sum_{m \in \mathbb{Z}} \int_{|t| \leq \pi} e^{i\left\langle x_{2 n-1}-y_{2 n-1}, \eta_{2 n-1}\right\rangle} \beta\left(\eta_{2 n-1}\right)\left(1-\chi\left(y_{2 n-1}\right)\right) \\
e^{i m y_{2 n-1}} e^{-i m t} u\left(x^{\prime}, t\right) d t d \eta_{2 n-1} d y_{2 n-1},
\end{array}
$$

where $x^{\prime}=\left(x_{1}, \ldots, x_{2 n-2}\right)$. Moreover, we can integrate by parts with respect to $\eta_{2 n-1}$ and conclude that

$$
R \equiv 0 \text { at } \Sigma^{-} \cap T^{*} D^{\prime}, \quad R \equiv 0 \text { at } \Sigma^{+} \cap T^{*} D^{\prime} .
$$

Now, we claim that

$$
Q_{\leq 0}^{(q)}(x, y)=R(x, y)+\frac{1}{(2 \pi)^{2 n-1}} \int e^{i\langle x-y, \eta\rangle} \beta\left(\eta_{2 n-1}\right) d \eta_{2 n-1} \text { on } D^{\prime}
$$

Let $u \in \Omega_{0}^{0, q}\left(D^{\prime}\right)$. From Fourier inversion formula, it is straightforward to see that

$$
\begin{aligned}
& \frac{1}{(2 \pi)^{2 n-1}} \int e^{i\langle x-y, \eta\rangle} \beta\left(\eta_{2 n-1}\right) u(y) d \eta_{2 n-1} \\
& =\frac{1}{(2 \pi)^{2}} \sum_{m \in \mathbb{Z}} \int_{|t| \leq \pi} e^{i\left\langle x_{2 n-1}-y_{2 n-1}, \eta_{2 n-1}\right\rangle} \beta\left(\eta_{2 n-1}\right) \\
& \quad \times \chi\left(y_{2 n-1}\right) e^{i m y_{2 n-1}} e^{-i m t} u\left(x^{\prime}, t\right) d t d \eta_{2 n-1} d y_{2 n-1} .
\end{aligned}
$$

From (6.48) and (6.51), we have

$$
\begin{aligned}
& \frac{1}{(2 \pi)^{2 n-1}} \int e^{i\langle x-y, \eta\rangle} \beta\left(\eta_{2 n-1}\right) u(y) d \eta_{2 n-1}+R u(x) \\
& =\frac{1}{(2 \pi)^{2}} \sum_{m \in \mathbb{Z}} \int_{|t| \leq \pi} e^{i\left\langle x_{2 n-1}-y 2 n-1, \eta_{2 n-1}\right\rangle} \beta\left(\eta_{2 n-1}\right) \\
& \quad \times e^{i m y_{2 n-1}} e^{-i m t} u\left(x^{\prime}, t\right) d t d \eta_{2 n-1} d y_{2 n-1} .
\end{aligned}
$$

From Fourier inversion formula and notice that for every $m \in \mathbb{Z}$,

$$
\int e^{i m y_{2 n-1}} e^{-i y_{2 n-1} \eta_{2 n-1}} d y_{2 n-1}=2 \pi \delta_{m}\left(\eta_{2 n-1}\right)
$$


where the integral above is defined as an oscillatory integral and $\delta_{m}$ is the Dirac measure at $m$ (see Chapter 7.2 in Hörmander [42]), (6.52) becomes

$$
\begin{aligned}
& \frac{1}{(2 \pi)^{2 n-1}} \int e^{i\langle x-y, \eta\rangle} \beta\left(\eta_{2 n-1}\right) u(y) d \eta_{2 n-1}+R u(x) \\
& =\frac{1}{2 \pi} \sum_{m \in \mathbb{Z}} \beta(m) e^{i x_{2 n-1} m} \int_{|t| \leq \pi} e^{-i m t} u\left(x^{\prime}, t\right) d t \\
& =\frac{1}{2 \pi} \sum_{m \in \mathbb{Z}, m \geq 0} e^{i x_{2 n-1} m} \int_{|t| \leq \pi} e^{-i m t} u\left(x^{\prime}, t\right) d t \\
& =Q_{\leq 0}^{(q)} u(x) .
\end{aligned}
$$

Here we used (6.47). The claim (6.50) follows. From (6.50) and (6.49), we conclude that

$$
\begin{aligned}
& Q_{\leq 0}^{(q)}(x, y)-\frac{1}{(2 \pi)^{2 n-1}} \int e^{i\langle x-y, \eta\rangle} \beta\left(\eta_{2 n-1}\right) d \eta_{2 n-1} \equiv 0 \text { at } \Sigma^{-} \cap T^{*} D^{\prime}, \\
& Q_{\leq 0}^{(q)}(x, y) \equiv 0 \text { at } \Sigma^{+} \cap T^{*} D^{\prime} .
\end{aligned}
$$

Moreover, it is straightforward to see that

$$
\frac{1}{(2 \pi)^{2 n-1}} \int e^{i\langle x-y, \eta\rangle}\left(\alpha\left(\eta_{2 n-1}\right)-\beta\left(\eta_{2 n-1}\right)\right) d \eta_{2 n-1} \equiv 0 \text { at } \Sigma^{-} \cap T^{*} D .
$$

From this observation, $(6.45)$ follows. The proof of $(6.46)$ is essentially the same as the proof of (6.45).

From Theorem 6.17, Theorem 6.18, Lemma 6.19 and Theorem 5.1, we get the following two results.

Theorem 6.20. Let $\left(X, T^{1,0} X\right)$ be a compact $C R$ manifold of dimension $2 n-1, n \geq 2$, with a transversal $C R S^{1}$ action and let $T \in \mathscr{C}^{\infty}(X, T X)$ be the real vector field induced by this $S^{1}$ action. We fix a T-rigid Hermitian metric $\langle\cdot \mid \cdot\rangle$ on $\mathbb{C} T X$ such that $T^{1,0} X \perp T^{0,1} X, T \perp$ $\left(T^{1,0} X \oplus T^{0,1} X\right),\langle T \mid T\rangle=1$ and $\langle u \mid v\rangle$ is real if $u, v$ are real tangent vectors and we take $m(x)$ to be the volume form induced by the given T-rigid Hermitian metric $\langle\cdot \mid \cdot\rangle$. Assume that $Z(q)$ fails but $Z(q-1)$ and $Z(q+1)$ hold at every point of $X$. Suppose that the Levi form is non-degenerate of constant signature $\left(n_{-}, n_{+}\right)$on an open canonical coordinate patch $D \Subset X$. Let $Q_{\leq 0}^{(q)}: L_{(0, q)}^{2}(X) \rightarrow L_{(0, q)}^{2}(X)$ be as in (6.14). Then,

$$
Q_{\leq 0}^{(q)} \Pi^{(q)} Q_{\leq 0}^{(q)} \equiv 0 \text { on } D \text { if } q \neq n_{-}
$$

and

$$
Q_{\leq 0}^{(q)} \Pi^{(q)} Q_{\leq 0}^{(q)}(x, y) \equiv \int_{0}^{\infty} e^{i \varphi_{-}(x, y) t} a(x, y, t) d t \text { on } D \text { if } q=n_{-},
$$

where $\varphi_{-} \in \mathscr{C}^{\infty}(D \times D)$ is as in Theorem 4.1 and $a(x, y, t) \in S_{\mathrm{cl}}^{n-1}\left(D \times D \times \mathbb{R}_{+}, T_{y}^{* 0, q} X \otimes T_{x}^{* 0, q} X\right)$ where with notations as in (3.7), (3.9), the leading term $a_{0}(x, y)$ of the expansion (2.16) of $a(x, y, t)$ satisfies

$$
a_{0}(x, x)=\frac{1}{2} \pi^{-n}\left|\operatorname{det} \mathcal{L}_{x}\right| \tau_{x, n_{-}}, \quad \forall x \in D .
$$

Similarly, we obtain the following. 
Theorem 6.21. Under the hypotheses of Theorem 6.20 assume that $Z(n-1-q)$ fails but $Z(n-2-q)$ and $Z(n-q)$ hold at every point of $X$. Suppose that the Levi form is non-degenerate of constant signature $\left(n_{-}, n_{+}\right)$on an open canonical coordinate patch $D \Subset X$. Let

$$
Q_{\geq 0}^{(q)}: L_{(0, q)}^{2}(X) \rightarrow L_{(0, q)}^{2}(X)
$$

be as in (6.15). Then,

$$
Q_{\geq 0}^{(q)} \Pi^{(q)} Q_{\geq 0}^{(q)} \equiv 0 \text { on } D \text { if } q \neq n_{+}
$$

and

$$
Q_{\geq 0}^{(q)} \Pi^{(q)} Q_{\geq 0}^{(q)}(x, y) \equiv \int_{0}^{\infty} e^{i \varphi_{+}(x, y) t} b(x, y, t) d t \text { on } D \text { if } q=n_{+},
$$

where $\varphi_{+} \in \mathscr{C}^{\infty}(D \times D)$ is as in Theorem 4.1 and $b(x, y, t) \in S_{\mathrm{cl}}^{n-1}\left(D \times D \times \mathbb{R}_{+}, T_{y}^{* 0, q} X \otimes T_{x}^{* 0, q} X\right)$, where with notations as in (3.7), (3.9), the leading term $b_{0}(x, y)$ of the expansion (2.16) of $b_{0}(x, y, t)$ satisfies

$$
b_{0}(x, x)=\frac{1}{2} \pi^{-n}\left|\operatorname{det} \mathcal{L}_{x}\right| \tau_{x, n_{+}}, \quad \forall x \in D .
$$

Kohn proved that if $X$ is any compact CR manifold and $Y(q)$ fails but $Y(q-1)$ and $Y(q+1)$ hold on $X$ then $\square_{b}^{(q)}$ has $L^{2}$ closed range (see [23]). By using Theorem 6.9, Theorem 6.10, Theorem 6.15 and Theorem 6.16, we can improve Kohn's result if $X$ admits a transversal CR $S^{1}$ action.

Definition 6.22. Given $q \in\{0, \ldots, n-1\}$, the Levi form is said to satisfy condition $W(q)$ at $p \in X$, if one of the following condition holds: (I) $Y(q)$ holds at $p$. (II) $Z(q), Z(n-2-q)$ and $Z(n-q)$ hold at $p$. (III) $Z(q-1), Z(q+1)$ and $Z(n-1-q)$ hold at $p$. (IV) $Y(q-1)$ and $Y(q+1)$ hold.

It is straightforward to see that if the Levi form is non-degenerate of constant signature on $X$ then for every $q \in\{0,1, \ldots, n-1\}, W(q)$ holds at every point of $X$. It is clear that if $Y(q-1)$ and $Y(q+1)$ hold at $p \in X$, or $Y(q)$ holds at $p$, then $W(q)$ holds at $p$. But it can happen that $W(q)$ holds at $p$ but $Y(q)$ fails at $p$ and $Y(q-1)$ or $Y(q+1)$ fail at $p$. For example, if the Levi form is non-degenerate of constant signature $\left(n_{-}, n_{+}\right)$at $p$ and $n_{+}=n_{-}+1$, then for $q=n_{-}, Z(q-1), Z(q+1)$ and $Z(n-1-q)$ hold at $p$. Thus, $W(q)$ holds at $p$ but $Y(q)$ and $Y(q+1)$ fail at $p$.

Theorem 6.23. Let $\left(X, T^{1,0} X\right)$ be a compact $C R$ manifold of dimension $2 n-1, n \geq 2$, with a transversal $C R S^{1}$ action and let $T \in \mathscr{C}^{\infty}(X, T X)$ be the real vector field induced by this $S^{1}$ action. We fix a T-rigid Hermitian metric $\langle\cdot \mid \cdot\rangle$ on $\mathbb{C} T X$ such that $T^{1,0} X \perp T^{0,1} X, T \perp$ $\left(T^{1,0} X \oplus T^{0,1} X\right),\langle T \mid T\rangle=1$ and $\langle u \mid v\rangle$ is real if $u, v$ are real tangent vectors and we take $m(x)$ to be the volume form induced by the given T-rigid Hermitian metric $\langle\cdot \mid \cdot\rangle$. Assume that $W(q)$ holds at every point of $X$. Then, $\square_{b}^{(q)}: \operatorname{Dom} \square_{b}^{(q)} \rightarrow L_{(0, q)}^{2}(X)$ has $L^{2}$ closed range. In particular, if the Levi form is non-degenerate of constant signature on $X$, then $\square_{b}^{(q)}: \operatorname{Dom} \square_{b}^{(q)} \rightarrow L_{(0, q)}^{2}(X)$ has $L^{2}$ closed range.

Proof. Since $W(q)$ holds at every point of $X$, from Theorem 6.9, Theorem 6.10, Theorem 6.15 and Theorem 6.16, we see that the operators

$$
\square_{b}^{(q)}: \operatorname{Dom} \square_{b}^{(q)} \cap \mathcal{B}_{\leq 0}^{0, q} \rightarrow \mathcal{B}_{\leq 0}^{0, q}, \quad \square_{b}^{(q)}: \operatorname{Dom} \square_{b}^{(q)} \cap \mathcal{B}_{\geq 0}^{0, q} \rightarrow \mathcal{B}_{\geq 0}^{0, q}
$$


have closed range. It is not difficult to see that this implies that $\square_{b}^{(q)}: \operatorname{Dom} \square_{b}^{(q)} \rightarrow L_{(0, q)}^{2}(X)$ has $L^{2}$ closed range. We leave the details to the reader.

Corollary 6.24. Under the same notations and assumptions used in Theorem 6.23, let $N^{(q)}$ : $L_{(0, q)}^{2}(X) \rightarrow \operatorname{Dom} \square_{b}^{(q)}$ be the partial inverse of $\square_{b}^{(q)}$. We assume that the Levi form is nondegenerate of constant signature $\left(n_{-}, n_{+}\right)$at each point of an open set $D \Subset X$. If $q \notin\left\{n_{-}, n_{+}\right\}$, then

$$
\Pi^{(q)} \equiv 0 \quad \text { and } \quad N^{(q)} \equiv A \quad \text { on } D,
$$

where $A \in L_{\frac{1}{2}, \frac{1}{2}}^{-1}\left(D, T^{* 0, q} X \otimes T^{* 0, q} X\right)$ is as in Theorem 4.1. If $q \in\left\{n_{-}, n_{+}\right\}$, then

$$
\Pi^{(q)} \equiv S_{-}+S_{+} \quad \text { and } \quad N^{(q)} \equiv G \quad \text { on } D,
$$

where $S_{-}, S_{+} \in L_{\frac{1}{2}, \frac{1}{2}}^{0}\left(D, T^{* 0, q} X \otimes T^{* 0, q} X\right)$ and $G \in L_{\frac{1}{2}, \frac{1}{2}}^{-1}\left(D, T^{* 0, q} X \otimes T^{* 0, q} X\right)$ are as in Theorem 4.1.

In particular, for any $C R$ submanifold in $\mathbb{C P}^{N}$ of the form (1.1), the associated Szegó kernel admits a full asymptotic expansion.

For hypersurfaces of type (1.1) of signature $(1, N-3)$, Biquard [8] studied the filling problem for small deformations of the CR structure.

From Corollary 6.24 and Theorem 6.23, we establish the global embeddablity for three dimensional compact strictly pseudoconvex CR manifolds with transversal CR $S^{1}$ actions (Theorem 1.13).

Theorem 6.25. Let $\left(X, T^{1,0} X\right)$ be a compact strictly pseudoconvex $C R$ manifold of dimension three with a transversal $C R S^{1}$ action. Then $X$ can be $C R$ embedded into $\mathbb{C}^{N}$, for some $N \in \mathbb{N}$.

Proof. Let $T \in \mathscr{C}^{\infty}(X, T X)$ be the real vector field induced by the given transversal CR $S^{1}$ action on $X$ and we fix a $T$-rigid Hermitian metric $\langle\cdot \mid \cdot\rangle$ on $\mathbb{C} T X$ such that $T^{1,0} X \perp T^{0,1} X$, $T \perp\left(T^{1,0} X \oplus T^{0,1} X\right),\langle T \mid T\rangle=1$ and $\langle u \mid v\rangle$ is real if $u, v$ are real tangent vectors and we take $m(x)$ to be the volume form induced by the given $T$-rigid Hermitian metric $\langle\cdot \mid \cdot\rangle$. We will use the same notations as before. From Theorem 6.23, we know that $\square_{b}^{(0)}:$ Dom $\square_{b}^{(q)} \rightarrow$ $L^{2}(X)$ has closed range. Let $N^{(0)}: L^{2}(X) \rightarrow$ Dom $\square_{b}^{(q)}$ be the partial inverse of $\square_{b}^{(q)}$. From Corollary 6.24, we have

$$
\begin{aligned}
& \square_{b}^{(0)} N^{(0)}+\Pi^{(0)}=I \text { on } L^{2}(X), \\
& N^{(0)} \square_{b}^{(0)}+\Pi^{(0)}=I \text { on } \operatorname{Dom} \square_{b}^{(0)}, \\
& N^{(0)} \in L_{\frac{1}{2}, \frac{1}{2}}^{-1}(X), \quad \Pi^{(0)} \in L_{\frac{1}{2}, \frac{1}{2}}^{0}(X) .
\end{aligned}
$$

From Kohn's result [52], in order to prove that $X$ can be CR embedded into $\mathbb{C}^{N}$, for some $N \in \mathbb{N}$, we only need to prove that $\bar{\partial}_{b}: \operatorname{Dom} \bar{\partial}_{b} \subset L^{2}(X) \rightarrow L_{(0,1)}^{2}(X)$ has closed range. Let $\bar{\partial}_{b} u_{j}=v_{j}, u_{j} \in \operatorname{Dom} \bar{\partial}_{b}, v_{j} \in L_{(0,1)}^{2}(X), j=1,2, \ldots$, with $v_{j} \rightarrow v \in L_{(0,1)}^{2}(X)$ as $j \rightarrow \infty$. We are going to prove that there is a $g \in \operatorname{Dom} \bar{\partial}_{b}$ such that $\bar{\partial}_{b} g=v$. We claim that for every $j=1,2, \ldots$,

$$
N^{(0)} \bar{\partial}_{b}^{*, f} v_{j} \in L_{(0,1)}^{2}(X) \text { and }\left(I-\Pi^{(0)}\right) u_{j}=N^{(0)} \bar{\partial}_{b}^{*, f} v_{j}
$$

where $\bar{\partial}_{b}^{*, f}$ is the formal adjoint of $\bar{\partial}_{b}$ (acting on distributions). Since $N^{(0)} \in L_{\frac{1}{2}, \frac{1}{2}}^{-1}(X)$, it is clearly that $N^{(0)} \bar{\partial}_{b}^{*, f} v_{j} \in L^{2}(X), \forall j$. Fix $j \in \mathbb{N}$. Let $f_{s} \in \mathscr{C}^{\infty}(X), s \in \mathbb{N}$, with $f_{s} \rightarrow u_{j}$ in $\mathscr{D}^{\prime}(X)$ 
as $s \rightarrow \infty$. From (6.58), we have

$$
N^{(0)} \bar{\partial}_{b}^{*, f} \bar{\partial}_{b} f_{s}=N^{(0)} \square_{b}^{(0)} f_{s}=\left(I-\Pi^{(0)}\right) f_{s} \rightarrow\left(I-\Pi^{(0)}\right) u_{j} \text { in } \mathscr{D}^{\prime}(X) \text { as } j \rightarrow \infty .
$$

Note that $N^{(0)} \bar{\partial}_{b}^{*, f} \bar{\partial}_{b} f_{s} \rightarrow N^{(0)} \bar{\partial}_{b}^{*, f} \bar{\partial}_{b} u_{j}=N^{(0)} \bar{\partial}_{b}^{*, f} v_{j}$ in $\mathscr{D}^{\prime}(X)$ as $j \rightarrow \infty$. From this observation and (6.60), the claim (6.59) follows. Since $N^{(0)} \in L_{\frac{1}{2}, \frac{1}{2}}^{-1}(X)$,

$$
N^{(0)} \bar{\partial}_{b}^{*, f}: L_{(0,1)}^{2}(X) \rightarrow L^{2}(X) \text { is continuous. }
$$

From (6.59) and (6.61), we conclude that

$$
\left(I-\Pi^{(0)}\right) u_{j}=N^{(0)} \bar{\partial}_{b}^{*, f} v_{j} \rightarrow N^{(0)} \bar{\partial}_{b}^{*, f} v=: u \text { in } L^{2}(X) .
$$

Thus, $\bar{\partial}_{b} u=v$ in the sense of distribution. Since $v \in L_{(0,1)}^{2}(X), u \in \operatorname{Dom} \bar{\partial}_{b}$. We have proved that $\bar{\partial}_{b}: \operatorname{Dom} \bar{\partial}_{b} \subset L^{2}(X) \rightarrow L_{(0,1)}^{2}(X)$ has closed range. The theorem follows.

Example 6.26 (Grauert tube). Let $M$ be a compact complex manifold endowed with a Hermitian metric $\Theta$ and associated Riemannian metric $g^{T M}$. We consider a Hermitian holomorphic line bundle $\left(L, h^{L}\right)$ on $M$. The Grauert tube associated to $\left(L, h^{L}\right)$ is the disc bundle $G=\left\{u \in L^{*},|u|_{h^{L^{*}}}<1\right\}$, with defining function $\varrho: L^{*} \rightarrow \mathbb{R}, \varrho=|u|_{h^{L^{*}}}^{2}-1$. The boundary $X=\partial G=\left\{u \in L^{*},|u|_{h^{L^{*}}}=1\right\}$ is the unit circle bundle in $L^{*}$. The Grauert tube was introduced by Grauert [35], one important application being the Kodaira embedding theorem for singular spaces.

Let $\nabla^{L}$ be the Chern connection on $\left(L, h^{L}\right)$ and let $R^{L}=\left(\nabla^{L}\right)^{2}$ be the Chern curvature. The Levi form of $\varrho$ restricted to the complex tangent plane of $X$ coincides with the pull-back of $\omega=\sqrt{-1} R^{L}$ through the canonical projection $\rho: X \rightarrow M$. Therefore, the signature of the Levi form of $\varrho$ coincides with the signature of the curvature form $\sqrt{-1} R^{L}$.

Note that $\rho: X \rightarrow M$ is a $S^{1}$-principal bundle and there exists a canonical $S^{1}$ action on $X$. The connection $\nabla^{L}$ on $L$ induces a connection on this $S^{1}$-principal bundle. Let $T^{H} X \subset T X$ be the corresponding horizontal bundle. Let us introduce the Riemannian metric $g^{T X}=$ $\rho^{*}\left(g^{T M}\right) \oplus d \vartheta^{2}$ on $T X=T^{H} X \oplus T S^{1}$. We will denote by $\bar{\partial}_{b}^{*}$ the formal adjoint of $\bar{\partial}_{b}$ with respect to this metric and form the Kohn-Laplacian $\square_{b}$. The operators $\bar{\partial}_{b}, \bar{\partial}_{b}^{*}$ and $\square_{b}^{(q)}$ commute with the action of $S^{1}$ on $X$.

Consider the space $\mathscr{C}^{\infty}(X)_{p}$ of smooth functions $f$ on $Y$ which transform under the action $(y, \vartheta) \mapsto e^{i \vartheta} y$ of $S^{1}$ according to the law

$$
f\left(e^{i \vartheta} y\right)=e^{i p \vartheta} f(y) .
$$

Then $\mathscr{C}^{\infty}(X)_{p}=B_{-p}^{0,0}(X)$, where $B_{m}^{0, q}(X)$ were defined in (6.7). Let us endow $\Omega^{\bullet \bullet \bullet}\left(M, L^{p}\right)$ with the $L^{2}$ inner product induced by $\Theta$ and $h^{L}$. There exists a natural isometry

$$
B_{-p}^{0,0}(X)=\mathscr{C}^{\infty}(X)_{p} \cong \Omega^{0,0}\left(M, L^{p}\right) .
$$

More generally, consider the space of sections $\Omega^{0, k}(X)_{p}$ which transform under the action of $S^{1}$ according to (6.62). Then $\Omega^{0, k}(X)_{p}=B_{-p}^{0, k}(X)$ is naturally isometric to the space $\Omega^{0, k}\left(X, L^{p}\right)$. In this way we obtain an interpretation of the spaces $B_{-p}^{0, q}(X), \mathcal{B}_{-p}^{0, q}(X)$ and of the projectors $Q_{\leq 0}, Q_{\geq 0}$ in terms of the sections of $L^{p}$. For more details on the relation of the Szegő projection and the Bergman kernel of $L^{p}$ one can consult [56, §1.5], [58, §3.2]. 


\section{SZEGŐ KERNEL ASYMPTOTIC EXPANSION ON WEAKLY PSEUDOCONVEX CR MANIFOLDS}

By using Theorem 1.9, we establish Szegő kernel asymptotic expansions on some weakly pseudoconvex CR manifolds. We will consider in Section 7.1 the case of boundaries of weakly pseudoconvex domains (corresponding to Corollary 1.15 (i)). We also give an application to the asymptotics of the Bergman kernel of a semi positive line bundle. In Section 7.2 we study some non-compact weakly pseudoconvex domains.

7.1. Compact pseudoconvex domains. Let $G$ be a relatively compact, weakly pseudoconvex domain, with smooth boundary $X$, in a complex manifold $G^{\prime}$ of dimension $n$. Then $X$ is a compact weakly pseudoconvex CR manifold of dimension $2 n-1$ with CR structure $T^{1,0} X:=T^{1,0} G^{\prime} \cap \mathbb{C} T X$.

Theorem 7.1. Let $G$ be a relatively compact domain in a complex manifold $G^{\prime}$ of dimension $n$, such that $G$ has smooth boundary $X=\partial G$, which is everywhere weakly pseudoconvex and strictly pseudoconvex on an open subset $D \subset X$. Fix $D_{0} \Subset D$. Assume that there exist a smooth strictly plurisubharmonic function defined in a neighborhood of $X$. Let $\phi \in \mathscr{C}^{\infty}\left(G^{\prime}\right)$ be a defining function of $G$, let $\langle\cdot \mid \cdot\rangle$ be a Hermitian metric on $G^{\prime}$ and let $v(x)$ be the induced volume form on $X$. Let $m(x)$ be a volume form on $X$ and consider the corresponding space $L^{2}(X)$. Then, the kernel of the Szegó projector $\Pi^{(0)}: L^{2}(X) \rightarrow \operatorname{ker} \bar{\partial}_{b}$ has the form

$$
\Pi^{(0)}(x, y) \equiv \int_{0}^{\infty} e^{i \varphi(x, y) t} s(x, y, t) d t \text { on } D_{0}
$$

where $\varphi(x, y) \in \mathscr{C}^{\infty}(U \times U)$ is an almost analytic extension of $\phi$ as in (1.3) to some neighbourhood $U$ be of $D_{0}$ in $G^{\prime}$, and $s(x, y, t) \in S_{\mathrm{cl}}^{n-1}\left(D \times D \times \mathbb{R}_{+}\right)$. Moreover the leading term $s_{0}(x, y)$ of the expansion (2.16) of $s_{0}(x, y, t)$ satisfies

$$
s_{0}(x, x)=\frac{1}{2} \pi^{-n} \frac{v(x)}{m(x)}\left|\operatorname{det} \mathcal{L}_{x}\right|, \quad \forall x \in D_{0},
$$

where $\mathcal{L}_{x}$ is the restriction of $\mathcal{L}_{x}(\phi)$ to the tangent space $T^{(1,0)} X$, $\left|\operatorname{det} \mathcal{L}_{x}\right|=\left|\mu_{1}(x)\right| \ldots\left|\mu_{n-1}(x)\right|$, with $\mu_{1}(x), \ldots, \mu_{n-1}(x)$ the eigenvalues of $\mathcal{L}_{x}$ with respect to $\langle\cdot \mid \cdot\rangle$.

Proof. By a theorem of Kohn [52, p. 543] we know that if $G$ meets the conditions in statement above, then Kohn's Laplacian $\square_{b}^{(0)}$ has $L^{2}$ closed range. For boundaries of pseudoconvex domains in $\mathbb{C}^{n}$ the closed range property was shown in [10,63]. By Theorem 1.14 we deduce that $\Pi^{(0)}$ is a complex Fourier integral operator on $D_{0}$ and $\Pi^{(0)}(x, y)$ has the form (7.1) with a phase function as in (3.2).

Fix $p \in D_{0}$ and take local coordinates $x=\left(x_{1}, x_{2}, \ldots, x_{2 n-1}\right)$ of $X$ defined in a small neighbourhood of $p$ in $D_{0}$ such that $x(p)=0$ and $\omega_{0}(p)=d x_{2 n-1}$. It is easy to see that $\frac{\partial \varphi}{\partial y_{2 n-1}}(0,0)=1=\frac{\partial \varphi_{-}}{\partial y_{2 n-1}}(0,0)$, where $\varphi_{-}$is as in Theorem 4.1. From the Malgrange preparation theorem [42, Theorem 7.57], we conclude that in some small neighbourhood of $(p, p)$ in $D_{0} \times D_{0}$, we can find $f(x, y), f_{1}(x, y) \in \mathscr{C}^{\infty}$ such that

$$
\begin{aligned}
& \varphi_{-}(x, y)=f(x, y)\left(y_{2 n-1}+h\left(x, y^{\prime}\right)\right), \\
& \varphi(x, y)=f_{1}(x, y)\left(y_{2 n-1}+h_{1}\left(x, y^{\prime}\right)\right)
\end{aligned}
$$

in some small neighbourhood of $(p, p)$ in $D_{0} \times D_{0}$, where $y^{\prime}=\left(y_{1}, \ldots, y_{2 n-2}\right), h, h_{1} \in \mathscr{C}^{\infty}$. It is not difficult to see that $y_{2 n-1}+h\left(x, y^{\prime}\right), y_{2 n-1}+h_{1}\left(x, y^{\prime}\right)$ satisfy (3.4), (3.5) and

$$
\bar{\partial}_{b}\left(y_{2 n-1}+h\left(x, y^{\prime}\right)\right), \bar{\partial}_{b}\left(y_{2 n-1}+h_{1}\left(x, y^{\prime}\right)\right)
$$


vanish to infinite order on $x=y$. From this observation, it is straightforward to check that $h\left(x, y^{\prime}\right)-h_{1}\left(x, y^{\prime}\right)$ vanishes to infinite order on $x=y$. We conclude that $\varphi$ and $\varphi_{-}$are equivalent. The theorem follows.

Theorem 7.2. Let $M$ be a projective manifold and let $L \rightarrow M$ be an ample line bundle. Let $h^{L}$ be a smooth Hermitian metric on $L$ such that $\sqrt{-1} R^{L}$ is semipositive. Consider the Grauert tube $G=\left\{v \in L^{*}:|v|_{h^{L^{*}}}<1\right\}, X=\partial G$ and $\rho: X \rightarrow M$ the projection. Then the Szegó projector $\Pi^{(0)}: L_{(0,0)}^{2}(X) \rightarrow \operatorname{ker} \bar{\partial}_{b}$ is a Fourier integral operator with complex phase on the set $\rho^{-1}(M(0))$, where $M(0) \subset M$ is the set where $\sqrt{-1} R^{L}$ is positive.

Proof. Since $L$ is ample, there exists a Hermitian metric $h_{0}^{L}$ on $L$ with positive curvature. The Levi form of the function $\varrho_{0}: L^{*} \rightarrow \mathbb{R}, \varrho=|u|_{h_{0}^{L^{*}}}^{2}$ is positive definite on the complex tangent space of any level set $\varrho_{0}=c>0$. It is easy to see that given any compact set $K \subset L^{*} \backslash 0$ we can modify $\varrho$ to construct a strictly plurisubharmonic on $K$. Therefore the Grauert tube $G$ fulfills the hypothesis of Theorem 7.1 .

Theorems 7.1 and 7.2 are based on closed range property for $\bar{\partial}_{b}$. Note that Donnelly [27] gave an example of a semipositive line bundle $L \rightarrow M$ which is positive at some point (i.e. $M(0) \neq \emptyset$ ), whose Grauert tube doesn't have the closed range property for $\bar{\partial}_{b}$.

An important application of the asymptotics of the Szego" kernel of the Grauert tube is the asymptotics of the Bergman kernel of the tensor powers of the bundle $L$. This was first achieved by Catlin [21] and Zelditch [66] for a positively curved metric $h^{L}$. We exemplify here such an application of Theorem 7.2 .

Consider a Hermitian metric $\Theta$ on $M$ and introduce the $L^{2}$ inner product on $\mathscr{C}^{\infty}\left(M, L^{p}\right)$ induced by the volume element $\Theta^{n} / n$ ! and the metric $h^{L^{p}}$ and denote by $L^{2}\left(M, L^{p}\right)$ the corresponding $L^{2}$ space. Let $P_{p}: L^{2}\left(M, L^{p}\right) \rightarrow H^{0}\left(M, L^{p}\right)$ be the orthogonal projection, called Bergman projection. Its kernel $P_{p}(\cdot, \cdot)$ is called the Bergman kernel. The restriction to the diagonal of $P_{p}(\cdot, \cdot)$ is denoted $P_{p}(\cdot)$ and is called the Bergman kernel function (or density). We refer the reader to the book [57] and to the survey [55] for a comprehensive study of the Bergman kernel and its applications.

Corollary 7.3. Let $M$ be a projective manifold of dimension $n$ and let $L \rightarrow M$ be an ample line bundle. Let $h^{L}$ be a smooth Hermitian metric on $L$ such that $\sqrt{-1} R^{L}$ is semipositive. Then the Bergman kernel function $P_{p}(\cdot)$ has the asymptotic expansion

$$
P_{p}(x) \sim \sum_{j=0}^{\infty} p^{n-j} b_{j}^{(0)}(x) \text { locally uniformly on } M(0),
$$

where $b_{j}^{(0)} \in \mathscr{C}^{\infty}(M(0)), j=0,1,2, \ldots$

Proof. The Bergman kernel $P_{p}$ and the Szegő kernel $\Pi^{(0)}$ are linked by the formula

$$
P_{p}(x)=\frac{1}{2 \pi} \int_{S^{1}} \Pi^{(0)}\left(e^{i \vartheta} y, y\right) e^{-i p \vartheta} d \vartheta
$$

where $x \in M$ and $y \in X$ satisfy $\rho(y)=x$, that is, $P_{p}(x)$ represent the Fourier coefficients of the distribution $\Pi^{(0)}(y, y)$. Since $\Pi^{(0)}$ is a Fourier integral operator on $\rho^{-1}(M(0))$ by Theorem 7.2, we deduce the asymptotics (7.2) exactly as in [21, 66] by applying the stationary phase method. 
Corollary 7.3 was obtained by different methods by Berman [5] in the case of a projective manifold $M$ and in [48, Theorem 1.10] for a general Hermitian manifold $M$.

7.2. Non-compact pseudoconvex domains. Now, we consider non-compact cases. By using Theorem 1.9, we will establish Szegő kernel asymptotic expansions on some non-compact CR manifolds. Let $\Gamma$ be a strictly pseudoconvex domain in $\mathbb{C}^{n-1}, n \geq 2$. Consider $X:=\Gamma \times \mathbb{R}$. Let $(z, t)$ be the coordinates of $X$, where $z=\left(z_{1}, \ldots, z_{n-1}\right)$ denote the coordinates of $\mathbb{C}^{n-1}$ and $t$ is the coordinate of $\mathbb{R}$. We write $z_{j}=x_{2 j-1}+i x_{2 j}, j=1, \ldots, n-1$. We also write $(z, t)=x=$ $\left(x_{1}, \ldots, x_{2 n-1}\right)$ and let $\eta=\left(\eta_{1}, \ldots, \eta_{2 n-1}\right)$ be the dual variables of $x$. Let $\mu(z) \in \mathscr{C}^{\infty}(\Gamma, \mathbb{R})$. We define $T^{1,0} X$ to be the space spanned by

$$
\left\{\frac{\partial}{\partial z_{j}}+i \frac{\partial \mu}{\partial z_{j}} \frac{\partial}{\partial t}, j=1, \ldots, n-1\right\} .
$$

Then $\left(X, T^{1,0} X\right)$ is a non-compact CR manifold of dimension $2 n-1$. We take a Hermitian metric $\langle\cdot \mid \cdot\rangle$ on the complexified tangent bundle $\mathbb{C} T X$ such that

$$
\left\{\frac{\partial}{\partial z_{j}}+i \frac{\partial \mu}{\partial z_{j}} \frac{\partial}{\partial t}, \frac{\partial}{\partial \bar{z}_{j}}-i \frac{\partial \mu}{\partial \bar{z}_{j}} \frac{\partial}{\partial t}, T:=\frac{\partial}{\partial t} ; j=1, \ldots, n-1\right\}
$$

is an orthonormal basis. The dual basis of the complexified cotangent bundle $\mathbb{C} T^{*} X$ is

$$
\left\{d z_{j}, d \bar{z}_{j},-\omega_{0}:=d t+\sum_{j=1}^{n-1}\left(-i \frac{\partial \mu}{\partial z_{j}} d z_{j}+i \frac{\partial \mu}{\partial \bar{z}_{j}} d \bar{z}_{j}\right) ; j=1, \ldots, n-1\right\} .
$$

The Levi form $\mathcal{L}_{p}$ of $X$ at $p \in X$ is given by

$$
\mathcal{L}_{p}=\sum_{j, \ell=1}^{n-1} \frac{\partial^{2} \mu}{\partial z_{j} \partial \bar{z}_{\ell}}(p) d z_{j} \wedge d \bar{z}_{\ell} .
$$

Now, we assume that

$$
\left(\frac{\partial^{2} \mu}{\partial z_{j} \partial \bar{z}_{\ell}}(z)\right)_{j, \ell=1}^{n-1} \geq 0, \quad \forall z \in \Gamma
$$

and take

$$
m(x):=e^{-2|z|^{2}} d x_{1} d x_{2} \ldots d x_{2 n-1}
$$

to be the volume form on $X$. Thus, $X$ is a weakly pseudoconvex CR manifold.

Take $\tau \in \mathscr{C}^{\infty}(\mathbb{R},[0,1])$ with $\tau=0$ on $\left.]-\infty, \frac{1}{4}\right], \tau=1$ on $\left[\frac{1}{2}, \infty[\right.$. We also write $\theta$ to denote the $t$ variable. Let $Q^{(0)}: \mathscr{C}_{0}^{\infty}(X) \rightarrow \mathscr{C}^{\infty}(X)$ be the operator given by

$$
Q^{(0)} u(z, t):=\frac{1}{2 \pi} \int e^{i\langle t-\theta, \eta\rangle} u(z, \theta) \tau(\eta) d \eta d \theta \in \mathscr{C}^{\infty}(X), \quad u(z, t) \in \mathscr{C}_{0}^{\infty}(X) .
$$

We can extend $Q^{(0)}$ to $L^{2}(X)$ such that

$$
\begin{aligned}
& Q^{(0)}: L^{2}(X) \rightarrow L^{2}(X) \text { is continuous, } \\
& \left\|Q^{(0)} u\right\| \leq\|u\|, \quad \forall u \in L^{2}(X), \\
& Q^{(0)} \in L_{\mathrm{cl}}^{0}(X), \\
& Q^{(0)} \equiv 0 \text { at } \Sigma^{+} \cap T^{*} D, \forall D \Subset X .
\end{aligned}
$$

We will prove that $\square_{b}^{(0)}$ has local $L^{2}$ closed range property on $X$ with respect to $Q^{(0)}$ under certain assumptions. More precisely, we have the following. 
Theorem 7.4. Let $\Gamma=\mathbb{C}^{n-1}$ or $\Gamma$ be a bounded strictly pseudoconvex domain in $\mathbb{C}^{n-1}$. Let $\mu \in \mathscr{C}^{\infty}\left(\Gamma^{\prime}\right)$, where $\Gamma^{\prime}$ is an open neighbourhood of $\bar{\Gamma}$ (if $\Gamma=\mathbb{C}^{n-1}$ this means just that $\mu \in$ $\left.\mathscr{C}^{\infty}\left(\mathbb{C}^{n-1}\right)\right)$. When $\Gamma=\mathbb{C}^{n-1}$, we assume that $\mu \geq 0$. Then

$$
\left\|Q^{(0)}\left(I-\Pi^{(0)}\right) u\right\|^{2} \leq C_{0}\left\|\bar{\partial}_{b} u\right\|^{2}, \forall u \in \mathscr{C}_{0}^{\infty}(X),
$$

where $C_{0}>0$ is a constant independent of $u$. In particular, $\square_{b}^{(0)}$ has local $L^{2}$ closed range on $X$ with respect to $Q^{(0)}$.

From Theorem 1.9, (7.7) and Theorem 7.4, we deduce

Theorem 7.5. With the notations and assumptions of Theorem 7.4 suppose that the matrix $\left(\frac{\partial^{2} \mu}{\partial z_{j} \partial \bar{z}_{\ell}}(x)\right)_{j, \ell=1}^{n-1}$ is positive definite on an open set $D \Subset X$. Then,

$$
Q^{(0)} \Pi^{(0)} Q^{(0)}(x, y) \equiv \int_{0}^{\infty} e^{i \varphi_{-}(x, y) t} a(x, y, t) d t, \text { on } D
$$

where $\varphi_{-}(x, y) \in \mathscr{C}^{\infty}(D \times D)$ is as in Theorem 4.1 and

$$
\begin{aligned}
& a(x, y, t) \in S_{\mathrm{cl}}^{n-1}\left(D \times D \times \mathbb{R}_{+}\right), \\
& a(x, y, t) \sim \sum_{j=0}^{\infty} a_{j}(x, y) t^{n-1-j} \quad \text { in } S_{1,0}^{n-1}\left(D \times D \times \mathbb{R}_{+}\right), \\
& a_{0}(x, x)=\frac{1}{2} \pi^{-n} \operatorname{det}\left(\frac{\partial^{2} \mu}{\partial z_{j} \partial \bar{z}_{\ell}}(x)\right)_{j, \ell=1}^{n-1}, \forall x \in D .
\end{aligned}
$$

We first introduce the partial Fourier transform $\mathcal{F}$ and the operator $Q^{(q)}$. Let $u \in \Omega_{0}^{0, q}(X)$. Put

$$
(\mathcal{F} u)(z, \eta)=\int_{\mathbb{R}} e^{-i \eta t} u(z, t) d t
$$

From Parseval's formula, we have

$$
\|\mathcal{F} u\|^{2}=\int_{X}|(\mathcal{F} u)(z, \eta)|^{2} d \eta d v(z)=2 \pi \int_{X}|u(z, t)|^{2} d t d v(z)=2 \pi\|u\|^{2},
$$

where $d v(z)=e^{-2|z|^{2}} d x_{1} d x_{2} \ldots d x_{2 n-2}$. Thus, we can extend the operator $\mathcal{F}$ to $L_{(0, q)}^{2}(X)$ and

$$
\begin{aligned}
& \mathcal{F}: L_{(0, q)}^{2}(X) \rightarrow L_{(0, q)}^{2}(X) \text { is continuous, } \\
& \|\mathcal{F} u\|=\sqrt{2 \pi}\|u\|, \quad \forall u \in L_{(0, q)}^{2}(X)
\end{aligned}
$$

For $u \in L_{(0, q)}^{2}(X)$, we call $\mathcal{F} u$ the partial Fourier transform of $u$ with respect to $t$.

Take $\tau \in \mathscr{C}^{\infty}(\mathbb{R},[0,1])$ with $\tau=0$ on $\left.]-\infty, \frac{1}{4}\right], \tau=1$ on $\left[\frac{1}{2}, \infty[\right.$. We also write $\theta$ to denote the $t$ variable. Let $Q^{(q)}: \Omega_{0}^{0, q}(X) \rightarrow \Omega^{0, q}(X)$ be the operator given by

$$
Q^{(q)} u(z, t):=\frac{1}{2 \pi} \int e^{i\langle t-\theta, \eta\rangle} u(z, \theta) \tau(\eta) d \eta d \theta \in \Omega^{0, q}(X), u(z, t) \in \Omega_{0}^{0, q}(X) .
$$


From Parseval's formula and (7.11), we have

$$
\begin{aligned}
\left\|Q^{(q)} u\right\|^{2} & =\frac{1}{4 \pi^{2}} \int_{X}\left|\int e^{i\langle t-\theta, \eta\rangle} u(z, \theta) \tau(\eta) d \eta d \theta\right|^{2} d v(z) d t \\
& =\frac{1}{4 \pi^{2}} \int_{X}\left|\int e^{i\langle t, \eta\rangle}(\mathcal{F} u)(z, \eta) \tau(\eta) d \eta\right|^{2} d v(z) d t \\
& =\frac{1}{2 \pi} \int|(\mathcal{F} u)(z, \eta)|^{2}|\tau(\eta)|^{2} d \eta d v(z) \\
& \leq \frac{1}{2 \pi} \int|(\mathcal{F} u)(z, \eta)|^{2} d \eta d v(z)=\|u\|^{2},
\end{aligned}
$$

where $u \in \Omega_{0}^{0, q}(X)$. Thus, we can extend $Q^{(q)}$ to $L_{(0, q)}^{2}(X)$ and

$$
\begin{aligned}
& Q^{(q)}: L_{(0, q)}^{2}(X) \rightarrow L_{(0, q)}^{2}(X) \text { is continuous, } \\
& \left\|Q^{(q)} u\right\| \leq\|u\|, \quad \forall u \in L_{(0, q)}^{2}(X)
\end{aligned}
$$

We need

Lemma 7.6. Let $u \in L_{(0, q)}^{2}(X)$. Then,

$$
\left(\mathcal{F} Q^{(q)} u\right)(z, \eta)=(\mathcal{F} u)(z, \eta) \tau(\eta) .
$$

Proof. Let $u_{j} \in \Omega_{0}^{0, q}(X), j=1,2, \ldots$, with $\lim _{j \rightarrow \infty}\left\|u_{j}-u\right\|=0$. From (7.15) and (7.12), we see that

$$
\mathcal{F} Q^{(q)} u_{j} \rightarrow \mathcal{F} Q^{(q)} u \text { in } L_{(0, q)}^{2}(X) \text { as } j \rightarrow \infty .
$$

From Fourier inversion formula, we have

$$
\left(\mathcal{F} Q^{(q)} u_{j}\right)(z, \eta)=\left(\mathcal{F} u_{j}\right)(z, \eta) \tau(\eta), \quad j=1, \ldots
$$

Note that $\left(\mathcal{F} u_{j}\right)(z, \eta) \tau(\eta) \rightarrow(\mathcal{F} u)(z, \eta) \tau(\eta)$ in $L_{(0, q)}^{2}(X)$ as $j \rightarrow \infty$. From this observation, (7.18) and (7.17), we obtain (7.16).

The following is straightforward. We omit the proofs.

Lemma 7.7. We have

$$
\begin{aligned}
& Q^{(q)}: \operatorname{Dom} \bar{\partial}_{b} \rightarrow \operatorname{Dom} \bar{\partial}_{b}, \quad q=0,1, \ldots, n-1, \\
& Q^{(q+1)} \bar{\partial}_{b}=\bar{\partial}_{b} Q^{(q)} \text { on } \operatorname{Dom} \bar{\partial}_{b}, \quad q=0,1, \ldots, n-2,
\end{aligned}
$$

and

$$
Q^{(q)} \Pi^{(q)}=\Pi^{(q)} Q^{(q)} \text { on } L_{(0, q)}^{2}(X) .
$$

Moreover, for $u \in \Omega_{0}^{0, q}(X)$, we have

$$
\bar{\partial}_{z}\left((\mathcal{F} u)(z, \eta) e^{\eta \mu(z)}\right) e^{-\eta \mu(z)}=\left(\mathcal{F} \bar{\partial}_{b} u\right)(z, \eta), \quad \forall(z, \eta) \in X,
$$

where $\mu \in \mathscr{C}^{\infty}(\Gamma, \mathbb{R})$ is as in the beginning of Section 7

We will study now the local $L^{2}$ closed range property for $\square_{b}^{(0)}$ with respect to $Q^{(0)}$. We pause and introduce some notations. Let $\Omega^{0, q}(\Gamma)$ be the space of all smooth $(0, q)$ forms on $\Gamma$ and let $\Omega_{0}^{0, q}(\Gamma)$ be the subspace of $\Omega^{0, q}(\Gamma)$ whose elements have compact support in $\Gamma$. We take the Hermitian metric $\langle\cdot \mid \cdot\rangle$ on $T^{* 0, q} \Gamma$ the bundle of $(0, q)$ forms of $\Gamma$ so that

$$
\left\{d \bar{z}_{j_{1}} \wedge d \bar{z}_{j_{2}} \wedge \ldots \wedge d \bar{z}_{j_{q}} ; 1 \leq j_{1}<j_{2} \ldots<j_{q} \leq n-1\right\}
$$


is an orthonormal basis. Let $\Upsilon \in \mathscr{C}^{\infty}(\Gamma, \mathbb{R})$ and let $(\cdot \mid \cdot)_{\Upsilon}$ be the $L^{2}$ inner product on $\Omega_{0}^{0, q}(\Gamma)$ given by

$$
(f \mid g)_{\Upsilon}=\int\langle f \mid g\rangle e^{-2 \Upsilon(z)} d \lambda(z), \quad f, g \in \Omega_{0}^{0, q}(\Gamma),
$$

where $d \lambda(z)=d x_{1} d x_{2} \ldots d x_{2 n-2}$. Let $L_{(0, q)}^{2}(\Gamma, \Upsilon)$ denote the completion of $\Omega_{0}^{0, q}(\Gamma)$ with respect to the inner product $(\cdot \mid \cdot)_{\Upsilon}$. We write $L^{2}(\Gamma, \Upsilon):=L_{(0,0)}^{2}(\Gamma, \Upsilon)$. Put

$$
H^{0}(\Gamma, \Upsilon):=\left\{f \in L^{2}(\Gamma, \Upsilon) ; \bar{\partial} f=0\right\}
$$

From now on, we assume that

$$
\left(\frac{\partial^{2} \mu}{\partial z_{j} \partial \bar{z}_{\ell}}(z)\right)_{j, \ell=1}^{n-1} \geq 0, \quad \forall z \in \Gamma
$$

and take

$$
m(x):=e^{-2|z|^{2}} d x_{1} d x_{2} \ldots d x_{2 n-2} d t=e^{-2|z|^{2}} d \lambda(z) d t
$$

be the volume form on $X$.

Now, suppose $\Gamma=\mathbb{C}^{n-1}$ or $\Gamma$ is a bounded strictly pseudoconvex domain in $\mathbb{C}^{n-1}$.

Proof of Theorem 7.4 for $\Gamma=\mathbb{C}^{n-1}$. Let $u \in \mathscr{C}_{0}^{\infty}(X)$. We consider $Q^{(0)}\left(I-\Pi^{(0)}\right) u$. In view of (7.20), we see that $Q^{(0)}\left(I-\Pi^{(0)}\right) u=\left(I-\Pi^{(0)}\right) Q^{(0)} u$. Put

$$
v(z, \eta)=\mathcal{F} Q^{(0)}\left(I-\Pi^{(0)}\right) u(z, \eta) e^{\eta \mu(z)} .
$$

From (7.15), (7.12) and (7.16), we see that $\int|v(z, \eta)|^{2} e^{-2 \eta \mu(z)-2|z|^{2}} d \lambda(z) d \eta<\infty$ and $v(z, \eta)=$ 0 if $\eta \notin \operatorname{Supp} \tau(\eta)$. From Fubini's Theorem and some elementary real analysis, we know that for every $\eta \in \mathbb{R}, v(z, \eta)$ is a measurable function of $z$ and for almost every $\eta \in \mathbb{R}$, $v(z, \eta) \in L^{2}\left(\Gamma, \eta \mu(z)+|z|^{2}\right)$ and for every $z \in \Gamma, v(z, \eta)$ is a measurable function of $\eta$ and for almost every $z \in \Gamma, \int|v(z, \eta)|^{2} d \eta<\infty$. Moreover, let $\beta \in L^{2}\left(\Gamma,|z|^{2}\right)$, then the function

$$
f(\eta):=\eta \mapsto \int v(z, \eta) \bar{\beta}(z) e^{-2 \eta \mu(z)-2|z|^{2}} d \lambda(z)
$$

is measurable and $f(\eta)$ is finite for almost every $\eta \in \mathbb{R}, f(\eta)=0$ if $\eta \notin \operatorname{Supp} \tau(\eta)$ and $f(\eta) \in L^{2}(\mathbb{R})$. We claim that

$$
\text { For almost every } \eta \in \overline{\mathbb{R}}_{+}, v(z, \eta) \in L^{2}\left(\Gamma, \eta \mu(z)+|z|^{2}\right) \text { and }
$$

$$
(v(z, \eta) \mid \beta)_{\eta \mu+|z|^{2}}=0, \quad \forall \beta \in H^{0}\left(\Gamma, \eta \mu(z)+|z|^{2}\right) .
$$

From the discussion after (7.23), we know that there is a measurable set $A_{0}$ in $\overline{\mathbb{R}}_{+}$with $\left|A_{0}\right|=0$ such that for every $\eta \notin A_{0}, v(z, \eta) \in L^{2}\left(\Gamma, \eta \mu(z)+|z|^{2}\right)$, where $\left|A_{0}\right|$ denote the Lebesgue measure of $A_{0}$. Since $\mu \geq 0,\left\{z^{\alpha} ; \alpha \in \mathbb{N}_{0}^{n-1}\right\}$ is a basis for $H^{0}\left(\mathbb{C}^{n-1}, \eta \mu(z)+|z|^{2}\right)$, for every $\eta \geq 0$. Fix $\alpha \in \mathbb{N}_{0}^{n-1}$. We consider

$$
f_{\alpha}(\eta)=\int v(z, \eta) \bar{z}^{\alpha} e^{-2 \eta \mu(z)-2|z|^{2}} d \lambda(z) .
$$

From the discussion after (7.23), we know that $f_{\alpha}(\eta) \in L^{2}(\mathbb{R})$. Fix $n \in \mathbb{N}$, put $h_{n}(\eta):=$ $\bar{f}_{\alpha}(\eta) 1_{[0, n]}(\eta)$. Then,

$$
\int_{0}^{n}\left|f_{\alpha}(\eta)\right|^{2} d \eta=\int f_{\alpha}(\eta) h_{n}(\eta) d \eta=\int v(z, \eta) \bar{z}^{\alpha} h_{n}(\eta) e^{-2 \eta \mu(z)-2|z|^{2}} d \lambda(z) d \eta
$$


Let $\beta_{\ell} \in \mathscr{C}_{0}^{\infty}(X), \ell=1,2, \ldots$, such that $\beta_{\ell} \rightarrow\left(I-\Pi^{(0)}\right) u$ in $L^{2}(X)$ as $\ell \rightarrow \infty$. From (7.12), (7.15), (7.16) and (7.25), we see that

$$
\lim _{\ell \rightarrow \infty} \int \mathcal{F} Q^{(0)} \beta_{\ell}(z, \eta) \bar{z}^{\alpha} h_{n}(\eta) e^{-\eta \mu(z)-2|z|^{2}} d \lambda(z) d \eta \rightarrow \int_{0}^{n}\left|f_{\alpha}(\eta)\right|^{2} d \eta .
$$

From (7.16) and Parseval's formula, we can check that

$$
\begin{aligned}
& \int \mathcal{F} Q^{(0)} \beta_{\ell}(z, \eta) \bar{z}^{\alpha} h_{n}(\eta) e^{-\eta \mu(z)-2|z|^{2}} d \lambda(z) d \eta \\
& =\int \mathcal{F} \beta_{\ell}(z, \eta) \tau(\eta) \bar{z}^{\alpha} h_{n}(\eta) e^{-\eta \mu(z)-2|z|^{2}} d \lambda(z) d \eta \\
& =\int \beta_{\ell}(z, t)\left(\int \bar{z}^{\alpha} h_{n}(\eta) \tau(\eta) e^{-\eta \mu(z)-i \eta t} d \eta\right) e^{-2|z|^{2}} d \lambda(z) d t \\
& \rightarrow \int\left(I-\Pi^{(0)}\right) u(z, t)\left(\int \bar{z}^{\alpha} h_{n}(\eta) \tau(\eta) e^{-\eta \mu(z)-i \eta t} d \eta\right) e^{-2|z|^{2}} d \lambda(z) d t \\
& \text { as } \ell \rightarrow \infty
\end{aligned}
$$

It is straightforward to check that the function

$$
\int z^{\alpha}(z) \bar{h}_{n}(\eta) \tau(\eta) e^{-\eta \mu(z)+i \eta t} d \eta \in \operatorname{Ker} \bar{\partial}_{b} \cap L^{2}(X) .
$$

Thus,

$$
\int\left(I-\Pi^{(0)}\right) u(z, t)\left(\int \bar{z}^{\alpha} h_{n}(\eta) \tau(\eta) e^{-\eta \mu(z)-i \eta t} d \eta\right) e^{-2|z|^{2}} d \lambda(z) d t=0 .
$$

From (7.28), (7.27) and (7.26), we conclude that $f_{\alpha}(\eta)=0$ almost everywhere. Thus, there is a measurable set $A_{\alpha} \supset A_{0}$ in $\overline{\mathbb{R}}_{+}$with $\left|A_{\alpha}\right|=0$ such that for every $\eta \notin A_{\alpha}$ we have $\left(v(z, \eta) \mid z^{\alpha}\right)_{\eta \mu+|z|^{2}}=0$. Put $A=\bigcup_{\alpha \in \mathbb{N}_{0}^{n-1}} A_{\alpha}$. Then, $|A|=0$. We conclude that for every $\eta \notin A, \eta \geq 0$,

$$
(v(z, \eta) \mid \beta)_{\eta \mu+|z|^{2}}=0, \quad \forall \beta \in H^{0}\left(\Gamma, \eta \mu+|z|^{2}\right) .
$$

The claim (7.24) follows.

Now, we can prove (7.8). Let $u \in \mathscr{C}_{0}^{\infty}(X)$. From (7.19) and (7.21), we have

$$
\begin{aligned}
& \bar{\partial}_{b} Q^{(0)}\left(I-\Pi^{(0)}\right) u=Q^{(1)} \bar{\partial}_{b} u \\
& \left(\mathcal{F} Q^{(1)} \bar{\partial}_{b} u\right)(z, \eta)=\bar{\partial}_{z}\left(\mathcal{F} Q^{(0)} u(z, \eta) e^{\eta \mu(z)}\right) e^{-\eta \mu(z)} .
\end{aligned}
$$

As before, we put $v(z, \eta)=\mathcal{F} Q^{(0)}\left(I-\Pi^{(0)}\right) u(z, \eta) e^{\eta \mu(z)}$ and set

$$
\bar{\partial}_{z}\left(\mathcal{F} Q^{(0)}\left(I-\Pi^{(0)}\right) u(z, \eta) e^{\eta \mu(z)}\right)=\bar{\partial}_{z} v(z, \eta)=: g(z, \eta) .
$$

It is easy to see that

$$
\begin{aligned}
& \bar{\partial}_{z} g(z, \eta)=0 \\
& g(z, \eta)=0 \text { if } \eta \notin \operatorname{Supp} \tau(\eta), \\
& \quad \int|g(z, \eta)|^{2} e^{-2 \eta \mu(z)-2|z|^{2}} d \lambda(z)<\infty, \quad \forall \eta \in \operatorname{Supp} \tau(\eta) .
\end{aligned}
$$


From (7.22), we see that there is a $C>0$ independent of $\eta \in \operatorname{Supp} \tau(\eta)$ such that

$$
\sum_{j, \ell=1}^{n-1} \frac{\partial^{2}\left(|z|^{2}+\eta \mu(z)\right)}{\partial z_{j} \partial \bar{z}_{\ell}}(z) w_{j} \bar{w}_{\ell} \geq C \sum_{j=1}^{n-1}\left|w_{j}\right|^{2}, \forall\left(w_{1}, \ldots, w_{n-1}\right) \in \mathbb{C}^{n-1}, z \in \Gamma, \eta \in \operatorname{Supp} \tau(\eta) .
$$

From (7.31) and Hörmander's $L^{2}$ estimates [41, Lemma 4.4.1], we conclude that for every $\eta \in \operatorname{Supp} \tau(\eta)$, we can find a $\beta_{\eta}(z) \in L_{(0,1)}^{2}\left(\Gamma, \eta \mu(z)+|z|^{2}\right)$ such that

$$
\bar{\partial}_{z} \beta_{\eta}(z)=g(z, \eta)
$$

and

$$
\int\left|\beta_{\eta}(z)\right|^{2} e^{-2 \eta \mu(z)-2|z|^{2}} d \lambda(z) \leq C \int|g(z, \eta)|^{2} e^{-2 \eta \mu(z)-2|z|^{2}} d \lambda(z) .
$$

In view of (7.24), we see that there is a measurable set $A$ in $\overline{\mathbb{R}}_{+}$with Lebesgue measure zero in $\mathbb{R}$ such that for every $\eta \notin A, \eta \geq 0, v(z, \eta) \perp H^{0}\left(\Gamma, \eta \mu(z)+|z|^{2}\right)$. Thus, for every $\eta \notin A, \eta \geq 0, v(z, \eta)$ has the minimum $L^{2}$ norm with respect to $(\cdot \mid \cdot)_{\eta \mu+|z|^{2}}$ of the solutions $\bar{\partial} \alpha=\bar{\partial}_{z} v(z, \eta)=g(z, \eta)$. From this observation and (7.33), we conclude that $\forall \eta \notin A$,

$$
\int|v(z, \eta)|^{2} e^{-2 \eta \mu(z)-2|z|^{2}} d \lambda(z) \leq C \int\left|\bar{\partial}_{z} v(z, \eta)\right|^{2} e^{-2 \eta \mu(z)-2|z|^{2}} d \lambda(z)
$$

Thus,

$$
\int|v(z, \eta)|^{2} e^{-2 \eta \mu(z)-2|z|^{2}} d \lambda(z) d \eta \leq C \int\left|\bar{\partial}_{z} v(z, \eta)\right|^{2} e^{-2 \eta \mu(z)-2|z|^{2}} d \lambda(z) d \eta .
$$

From the definition of $v(z, \eta),(7.12),(7.29)$ and (7.15), it is straightforward to see that

$$
\begin{aligned}
& \int|v(z, \eta)|^{2} e^{-2 \eta \mu(z)-2|z|^{2}} d \lambda(z) d \eta \\
& =(2 \pi) \int\left|Q^{(0)}\left(I-\Pi^{(0)}\right) u(z, t)\right|^{2} e^{-2|z|^{2}} d \lambda(z) d t
\end{aligned}
$$

and

$$
\begin{aligned}
& \int\left|\bar{\partial}_{z} v(z, \eta)\right|^{2} e^{-2 \eta \mu(z)-2|z|^{2}} d \lambda(z) d \eta \\
& =(2 \pi) \int\left|Q^{(1)} \bar{\partial}_{b} u(z, t)\right|^{2} e^{-2|z|^{2}} d \lambda(z) d t \\
& \leq(2 \pi) \int\left|\bar{\partial}_{b} u(z, t)\right|^{2} e^{-2|z|^{2}} d \lambda(z) d t .
\end{aligned}
$$

From (7.35), (7.36) and (7.37), we conclude that

$$
\begin{aligned}
\left\|Q^{(0)}\left(I-\Pi^{(0)}\right) u\right\|^{2} & =\int\left|Q^{(0)}\left(I-\Pi^{(0)}\right) u(z, t)\right|^{2} e^{-2|z|^{2}} d \lambda(z) d t \\
& \leq C \int\left|\bar{\partial}_{b} u(z, t)\right|^{2} e^{-2|z|^{2}} d \lambda(z) d t=C\left\|\bar{\partial}_{b} u\right\|^{2} .
\end{aligned}
$$

Theorem 7.4 for $\Gamma=\mathbb{C}^{n-1}$ follows.

Now, we consider the case when $\Gamma$ is a bounded strictly pseudoconvex domain in $\mathbb{C}^{n-1}$. 
Proof of Theorem 7.4 for $\Gamma$ a bounded strictly pseudoconvex domain in $\mathbb{C}^{n-1}$. Let $u \in \mathscr{C}_{0}^{\infty}(X)$. We have

$$
\begin{aligned}
& \bar{\partial}_{b} Q^{(0)}\left(I-\Pi^{(0)}\right) u=Q^{(1)} \bar{\partial}_{b} u \\
& \left(\mathcal{F} Q^{(1)} \bar{\partial}_{b} u\right)(z, \eta)=\bar{\partial}_{z}\left(\mathcal{F} Q^{(0)} u(z, \eta) e^{\eta \mu(z)}\right) e^{-\eta \mu(z)} .
\end{aligned}
$$

As before, we put $v(z, \eta)=\mathcal{F} Q^{(0)}\left(I-\Pi^{(0)}\right) u(z, \eta) e^{\eta \mu(z)}$ and set

$$
\bar{\partial}_{z}\left(\mathcal{F} Q^{(0)}\left(I-\Pi^{(0)}\right) u(z, \eta) e^{\eta \mu(z)}\right)=\bar{\partial}_{z} v(z, \eta)=: g(z, \eta) .
$$

Then,

$$
\begin{aligned}
& \bar{\partial}_{z} g(z, \eta)=0 \\
& g(z, \eta)=0 \text { if } \eta \notin \operatorname{Supp} \tau(\eta), \\
& \quad \int|g(z, \eta)|^{2} e^{-2 \eta \mu(z)-2|z|^{2}} d \lambda(z)<\infty, \quad \forall \eta \in \operatorname{Supp} \tau(\eta) .
\end{aligned}
$$

From (7.31) and Hörmander's $L^{2}$ estimates [41, Lemma 4.4.1], we conclude that for every $\eta \in \operatorname{Supp} \tau(\eta)$, we can find a $\beta_{\eta}(z) \in L_{(0,1)}^{2}\left(\Gamma, \eta \mu(z)+|z|^{2}\right)$ such that

$$
\bar{\partial}_{z} \beta_{\eta}(z)=g(z, \eta)
$$

and

$$
\int\left|\beta_{\eta}(z)\right|^{2} e^{-2 \eta \mu(z)-2|z|^{2}} d \lambda(z) \leq C \int|g(z, \eta)|^{2} e^{-2 \eta \mu(z)-2|z|^{2}} d \lambda(z)
$$

where $C>0$ is a constant independent of $\eta, g(z, \eta)$ and $\beta_{\eta}(z)$. Moreover, since $g(z, \eta)$ is smooth, it is well-known that $\beta_{\eta}(z)$ can be taken to be dependent smoothly on $\eta$ and $z$ (see the proof of [6, Lemma 2.1]). Take $\chi(\eta) \in \mathscr{C}^{\infty}(\mathbb{R},[0,1])$ with $\chi=0$ if $|\eta| \geq 1$ and $\chi=1$ if $|\eta| \leq \frac{1}{2}$. For $j=1,2, \ldots$, set $\chi_{j}(\eta)=\chi\left(\frac{\eta}{j}\right)$. Put

$$
\alpha_{j}(z, t)=\frac{1}{2 \pi} \int \beta_{\eta}(z) \chi_{j}(\eta) e^{-\eta \mu(z)} e^{i \eta t} d \eta \in \mathscr{C}^{\infty}(X) .
$$

From (7.41), we have

$$
\begin{aligned}
& \left\|\alpha_{j}-\alpha_{k}\right\|^{2} \\
& =\frac{1}{4 \pi^{2}} \int\left|\int \beta_{\eta}(z)\left(\chi_{j}(\eta)-\chi_{k}(\eta)\right) e^{-\eta \mu(z)} e^{i \eta t}(\eta) d \eta\right|^{2} e^{-2|z|^{2}} d \lambda(z) d t \\
& \leq \frac{1}{4 \pi^{2}} \iint\left|\beta_{\eta}(z)\right|^{2}\left|\chi_{j}(\eta)-\chi_{k}(\eta)\right|^{2} e^{-2 \eta \mu(z)-2|z|^{2}} d \lambda(z) d \eta \\
& \leq C_{0} \iint|g(z, \eta)|^{2}\left|\chi_{j}(\eta)-\chi_{k}(\eta)\right|^{2} e^{-2 \eta \mu(z)-2|z|^{2}} d \lambda(z) d \eta \\
& \rightarrow 0 \text { as } j, k \rightarrow \infty,
\end{aligned}
$$

where $C_{0}>0$ is a constant independent of $j, k, \beta_{\eta}(z)$ and $g(z, \eta)$. Thus, $\alpha_{j} \rightarrow \alpha$ in $L^{2}(X)$, for some $\alpha \in L^{2}(X)$. Moreover, we can repeat the procedure above with minor change and deduce that

$$
\begin{aligned}
\|\alpha\|^{2} & \leq C_{0} \iint|g(z, \eta)|^{2} e^{-2 \eta \mu(z)-2|z|^{2}} d \lambda(z) d \eta \\
& \leq C_{0}(2 \pi)\left\|Q^{(1)} \bar{\partial}_{b} u\right\|^{2} \leq C_{1}\left\|\bar{\partial}_{b} u\right\|^{2},
\end{aligned}
$$


where $C_{0}>0$ is the constant as in (7.42) and $C_{1}=C_{0}(2 \pi)$. Furthermore, it is straightforward to see that

$$
\bar{\partial}_{b} \alpha(z, t)=Q^{(1)} \bar{\partial}_{b} u(z, t) .
$$

From (7.43) and (7.44), we conclude that $\bar{\partial}_{b} \alpha(z, t)=Q^{(1)} \bar{\partial}_{b} u(z, t)$ and $\|\alpha\|^{2} \leq C_{1}\left\|\bar{\partial}_{b} u\right\|^{2}$. Since $\left(I-\Pi^{(0)}\right) Q^{(0)} u$ has the minimum $L^{2}$ norm of the solutions of $\bar{\partial}_{b} f=Q^{(1)} \bar{\partial}_{b} u(z, t)$, we conclude that

$$
\left\|\left(I-\Pi^{(0)}\right) Q^{(0)} u\right\|^{2}=\left\|Q^{(0)}\left(I-\Pi^{(0)}\right) u\right\|^{2} \leq\|\alpha\|^{2} \leq C_{1}\left\|\bar{\partial}_{b} u\right\|^{2} .
$$

Theorem 7.4 follows.

Proof of Theorem 7.5 Recall that $\omega_{0}=-d t+\sum_{j=1}^{n-1}\left(i \frac{\partial \mu}{\partial z_{j}} d z_{j}-i \frac{\partial \mu}{\partial \bar{z}_{j}} d \bar{z}_{j}\right)$. Thus,

$$
\begin{aligned}
& \Sigma^{+}=\left\{(x, \eta) \in T^{*} X ; \eta=-\lambda d x_{2 n-1}+\lambda \sum_{j=1}^{n-1}\left(\frac{\partial \mu}{\partial x_{2 j}}(z) d x_{2 j-1}-\frac{\partial \mu}{\partial x_{2 j-1}}(z) d x_{2 j}\right), \lambda>0\right\}, \\
& \Sigma^{-}=\left\{(x, \eta) \in T^{*} X ; \eta=-\lambda d x_{2 n-1}+\lambda \sum_{j=1}^{n-1}\left(\frac{\partial \mu}{\partial x_{2 j}}(z) d x_{2 j-1}-\frac{\partial \mu}{\partial x_{2 j-1}}(z) d x_{2 j}\right), \lambda<0\right\} .
\end{aligned}
$$

Note that

$$
Q^{(0)}(x, y)=\int e^{i\langle x-y, \eta\rangle} \tau\left(\eta_{2 n-1}\right) d \eta
$$

where $\tau \in \mathscr{C}^{\infty}(\mathbb{R},[0,1])$ with $\tau=0$ on $\left.]-\infty, \frac{1}{4}\right], \tau=1$ on $\left[\frac{1}{2}, \infty[\right.$. From this observation and (7.45), we conclude that

$$
Q^{(0)} \equiv 0 \text { at } \Sigma^{+} \cap T^{*} D, \forall D \Subset X .
$$

From Theorem 1.9, Theorem 7.4 and $(\mathbf{7 . 4 6})$, we get Theorem 7.5 ,

\section{Proof of Theorem 5.4}

We introduce some notations from semi-classical analysis.

Definition 8.1. Let $W$ be an open set in $\mathbb{R}^{N}$. We define the space of symbols

$$
S(1)=S(1 ; W)=\left\{a \in \mathscr{C}^{\infty}(W) ; \forall \alpha \in \mathbb{N}_{0}^{N} \exists C_{\alpha}>0:\left|\partial_{x}^{\alpha} a(x)\right| \leq C_{\alpha} \text { on } W\right\} .
$$

If $a=a(x, k)$ depends on $k \in] 1, \infty$ [, we say that $a(x, k) \in S_{\mathrm{loc}}(1 ; W)=S_{\mathrm{loc}}(1)$ if $\chi(x) a(x, k)$ uniformly bounded in $S(1)$ when $k$ varies in ]1, $\infty$ [, for any $\chi \in \mathscr{C}_{0}^{\infty}(W)$. For $m \in \mathbb{R}$, we put $S_{\mathrm{loc}}^{m}(1 ; W)=S_{\mathrm{loc}}^{m}(1)=k^{m} S_{\mathrm{loc}}(1)$. If $a_{j} \in S_{\mathrm{loc}}^{m_{j}}(1), m_{j} \searrow-\infty$, we say that $a \sim \sum_{j=0}^{\infty} a_{j}$ in $S_{\text {loc }}^{m_{0}}(1)$ if $a-\sum_{j=0}^{N_{0}} a_{j} \in S_{\text {loc }}^{m_{N_{0}+1}}(1)$ for every $N_{0}$. For a given sequence $a_{j}$ as above, we can always find such an asymptotic sum $a$ and $a$ is unique up to an element in $S_{\text {loc }}^{-\infty}(1)=$ $S_{\text {loc }}^{-\infty}(1 ; W):=\cap_{m} S_{\text {loc }}^{m}(1)$. We say that $a(x, k) \in S_{\text {loc }}^{m_{0}}(1)$ is a classical symbol on $W$ of order $m_{0}$ if

$$
a(x, k) \sim \sum_{j=0}^{\infty} k^{m_{0}-j} a_{j}(x) \text { in } S_{\mathrm{loc}}^{m_{0}}(1), a_{j}(x) \in S_{\mathrm{loc}}(1), j=0,1 \ldots
$$

The set of all classical symbols on $W$ of order $m_{0}$ is denoted by $S_{\mathrm{loc}, \mathrm{cl}}^{m_{0}}(1)=S_{\mathrm{loc}, \mathrm{cl}}^{m_{0}}(1 ; W)$.

Let $E$ be a vector bundle over a smooth paracompact manifold $Y$. We extend the definitions above to the space of smooth sections of $E$ over $Y$ in the natural way and we write $S_{\mathrm{loc}}^{m}(1 ; Y, E)$ and $S_{\mathrm{loc}, \mathrm{cl}}^{m}(1 ; Y, E)$ to denote the corresponding spaces. 
A $k$-dependent continuous operator $A_{k}: \mathscr{C}_{0}^{\infty}(W, E) \rightarrow \mathscr{D}^{\prime}(W, F)$ is called $k$-negligible (on $W)$ if $A_{k}$ is smoothing and the kernel $A_{k}(x, y)$ of $A_{k}$ satisfies $\left|\partial_{x}^{\alpha} \partial_{y}^{\beta} A_{k}(x, y)\right|=O\left(k^{-N}\right)$ locally uniformly on every compact set in $W \times W$, for all multi-indices $\alpha, \beta$ and all $N \in \mathbb{N} . A_{k}$ is $k$-negligible if and only if

$$
A_{k}=O\left(k^{-N^{\prime}}\right): H_{\mathrm{comp}}^{s}(W, E) \rightarrow H_{\mathrm{loc}}^{s+N}(W, F)
$$

for all $N, N^{\prime} \geq 0$ and $s \in \mathbb{Z}$. Let $C_{k}: \mathscr{C}_{0}^{\infty}(W, E) \rightarrow \mathscr{D}^{\prime}(W, F)$ be another $k$-dependent continuous operator. We write $A_{k} \equiv C_{k} \bmod O\left(k^{-\infty}\right)$ (on $W$ ) or $A_{k}(x, y) \equiv C_{k}(x, y) \bmod O\left(k^{-\infty}\right)$ (on $W$ ) if $A_{k}-C_{k}$ is $k$-negligible on $W$.

Now, we prove Theorem [5.4. We will use the same notations and assumptions in Theorem 5.4. Fix $p \in D$. Take local coordinates $x=\left(x_{1}, \ldots, x_{2 n-1}\right)$ defined in some small neighbourhood of $p$ such that $x(p)=0$ and $\omega_{0}(p)=d x_{2 n-1}$. Since $\left.d_{y} \varphi(x, y)\right|_{x=y}=\left.d_{y} \varphi_{-}(x, y)\right|_{x=y}=$ $\omega_{0}(x)$, we have $\frac{\partial \varphi}{\partial y_{2 n-1}}(p, p)=\frac{\partial \varphi_{-}}{\partial y_{2 n-1}}(p, p)=1$. From this observation and the Malgrange preparation theorem [42, Theorem 7.57], we conclude that in some small neighbourhood of $(p, p)$, we can find $f(x, y), f_{1}(x, y) \in \mathscr{C}^{\infty}$ such that

$$
\begin{aligned}
& \varphi_{-}(x, y)=f(x, y)\left(y_{2 n-1}+h\left(x, y^{\prime}\right)\right), \\
& \varphi(x, y)=f_{1}(x, y)\left(y_{2 n-1}+h_{1}\left(x, y^{\prime}\right)\right)
\end{aligned}
$$

in some small neighbourhood of $(p, p)$, where $y^{\prime}=\left(y_{1}, \ldots, y_{2 n-2}\right)$. For simplicity, we assume that (8.2) hold on $D \times D$. It is clearly that $\varphi_{-}(x, y)$ and $y_{2 n-1}+h\left(x, y^{\prime}\right)$ are equivalent in the sense of Melin-Sjöstrand [60], $\varphi(x, y)$ and $y_{2 n-1}+h_{1}\left(x, y^{\prime}\right)$ are equivalent in the sense of MelinSjöstrand [60], we may assume that $\varphi_{-}(x, y)=y_{2 n-1}+h\left(x, y^{\prime}\right)$ and $\varphi(x, y)=y_{2 n-1}+h_{1}\left(x, y^{\prime}\right)$. Fix $x_{0} \in D$. We are going to prove that $h\left(x, y^{\prime}\right)-h_{1}\left(x, y^{\prime}\right)$ vanishes to infinite order at $\left(x_{0}, x_{0}\right)$. Note that $S_{-} \circ S_{-} \equiv S_{-}$. From this observation and Lemma 5.3, it is straightforward to see that

$$
\int_{0}^{\infty} e^{i\left(y_{2 n-1}+h\left(x, y^{\prime}\right)\right) t} s_{-}(x, y, t) d t \equiv \int_{0}^{\infty} e^{i\left(y_{2 n-1}+h_{1}\left(x^{\prime}, y\right)\right) t} a(x, y, t) d t \text { on } D
$$

where $s_{-}(x, y, t), a(x, y, t) \in S_{\mathrm{cl}}^{n-1}\left(D \times D \times \mathbb{R}_{+}, T^{* 0, q} X \otimes T^{* 0, q} X\right)$ are as in (2.15). Put

$$
x_{0}=\left(x_{0}^{1}, x_{0}^{2}, \ldots, x_{0}^{2 n-1}\right), \quad x_{0}^{\prime}=\left(x_{0}^{1}, \ldots, x_{0}^{2 n-2}\right) .
$$

Take $\tau \in \mathscr{C}_{0}^{\infty}\left(\mathbb{R}^{2 n-1}\right), \tau_{1} \in \mathscr{C}_{0}^{\infty}\left(\mathbb{R}^{2 n-2}\right), \chi \in \mathscr{C}_{0}^{\infty}(\mathbb{R})$ so that $\tau=1$ near $x_{0}, \tau_{1}=1$ near $x_{0}^{\prime}$, $\chi=1$ near $x_{0}^{2 n-1}$ and $\operatorname{Supp} \tau \Subset D$, Supp $\tau_{1} \times \operatorname{Supp} \chi \Subset D^{\prime} \times \operatorname{Supp} \chi \Subset D$, where $D^{\prime}$ is an open neighbourhood of $x_{0}^{\prime}$ in $\mathbb{R}^{2 n-2}$. For each $k>0$, we consider the distributions

$$
\begin{aligned}
& A_{k}: u \mapsto \int_{0}^{\infty} e^{i\left(y_{2 n-1}+h\left(x, y^{\prime}\right)\right) t-i k y_{2 n-1}} \tau(x) s_{-}(x, y, t) \tau_{1}\left(y^{\prime}\right) \chi\left(y_{2 n-1}\right) u\left(y^{\prime}\right) d y d t \\
& B_{k}: u \mapsto \int_{0}^{\infty} e^{i\left(y_{2 n-1}+h_{1}\left(x, y^{\prime}\right)\right) t-i k y_{2 n-1}} \tau(x) a(x, y, t) \tau_{1}\left(y^{\prime}\right) \chi\left(y_{2 n-1}\right) u\left(y^{\prime}\right) d y d t
\end{aligned}
$$


for $u \in \mathscr{C}_{0}^{\infty}\left(D^{\prime}, T^{* 0, q} X\right)$. By using the stationary phase formula of Melin-Sjöstrand [60], we can show that (cf. the proof of [48, Theorem 3.12]) $A_{k}$ and $B_{k}$ are smoothing operators and

$$
\begin{aligned}
& A_{k}\left(x, y^{\prime}\right) \equiv e^{i k h\left(x, y^{\prime}\right)} g\left(x, y^{\prime}, k\right) \quad \bmod O\left(k^{-\infty}\right), \\
& B_{k}\left(x, y^{\prime}\right) \equiv e^{i k h_{1}\left(x, y^{\prime}\right)} b\left(x, y^{\prime}, k\right) \quad \bmod O\left(k^{-\infty}\right), \\
& g\left(x, y^{\prime}, k\right), b\left(x, y^{\prime}, k\right) \in S_{\mathrm{loc}, \mathrm{cl}}^{n-1}\left(1 ; D^{\prime} \times D, T^{* 0, q} X \otimes T^{* 0, q} X\right), \\
& g\left(x, y^{\prime}, k\right) \sim \sum_{j=0}^{\infty} g_{j}\left(x, y^{\prime}\right) k^{n-1-j} \text { in } S_{\mathrm{loc}}^{n-1}\left(1 ; D^{\prime} \times D, T^{* 0, q} X \otimes T^{* 0, q} X\right), \\
& b\left(x, y^{\prime}, k\right) \sim \sum_{j=0}^{\infty} b_{j}\left(x, y^{\prime}\right) k^{n-1-j} \text { in } S_{\mathrm{loc}}^{n-1}\left(1 ; D^{\prime} \times D, T^{* 0, q} X \otimes T^{* 0, q} X\right), \\
& g_{j}\left(x, y^{\prime}\right), b_{j}\left(x, y^{\prime}\right) \in \mathscr{C}^{\infty}\left(D \times D^{\prime}, T_{y^{\prime}}^{* 0, q} X \otimes T_{x}^{* 0, q} X\right), \quad j=0,1, \ldots, \\
& g_{0}\left(x_{0}, x_{0}^{\prime}\right) \neq 0, \quad b_{0}\left(x_{0}, x_{0}^{\prime}\right) \neq 0 .
\end{aligned}
$$

Since

$$
\int_{0}^{\infty} e^{i\left(y_{2 n-1}+h\left(x, y^{\prime}\right)\right) t} s_{-}(x, y, t) d t-\int_{0}^{\infty} e^{i\left(y_{2 n-1}+h_{1}\left(x, y^{\prime}\right)\right) t} a(x, y, t) d t
$$

is smoothing, by using integration by parts with respect to $y_{2 n-1}$, it is easy to see that $A_{k}-B_{k} \equiv$ $0 \bmod O\left(k^{-\infty}\right)$ (see [48, Section 3]). Thus,

$$
\begin{aligned}
& e^{i k h\left(x, y^{\prime}\right)} g\left(x, y^{\prime}, k\right)=e^{i k h_{1}\left(x, y^{\prime}\right)} b\left(x, y^{\prime}, k\right)+F_{k}\left(x, y^{\prime}\right), \\
& F_{k}\left(x, y^{\prime}\right) \equiv 0 \quad \bmod O\left(k^{-\infty}\right) .
\end{aligned}
$$

Now, we are ready to prove that $h\left(x, y^{\prime}\right)-h_{1}\left(x, y^{\prime}\right)$ vanishes to infinite order at $\left(x_{0}, x_{0}^{\prime}\right)$. We assume that there exist $\alpha_{0} \in \mathbb{N}_{0}^{2 n-1}, \beta_{0} \in \mathbb{N}_{0}^{2 n-2},\left|\alpha_{0}\right|+\left|\beta_{0}\right| \geq 1$ such that

$$
\left.\partial_{x}^{\alpha_{0}} \partial_{y^{\prime}}^{\beta_{0}}\left(i h\left(x, y^{\prime}\right)-i h_{1}\left(x, y^{\prime}\right)\right)\right|_{\left(x_{0}, x_{0}^{\prime}\right)}=C_{\alpha_{0}, \beta_{0}} \neq 0
$$

and

$$
\left.\partial_{x}^{\alpha} \partial_{y^{\prime}}^{\beta}\left(i h\left(x, y^{\prime}\right)-i h_{1}\left(x, y^{\prime}\right)\right)\right|_{\left(x_{0}, x_{0}^{\prime}\right)}=0 \text { if }|\alpha|+|\beta|<\left|\alpha_{0}\right|+\left|\beta_{0}\right| .
$$

From (8.6), we have

$$
\begin{aligned}
& \left.\partial_{x}^{\alpha_{0}} \partial_{y}^{\beta_{0}}\left(e^{i k h\left(x, y^{\prime}\right)-i k h_{1}\left(x, y^{\prime}\right)} g\left(x, y^{\prime}, k\right)-b(x, y, k)\right)\right|_{\left(x_{0}, x_{0}^{\prime}\right)} \\
& =-\left.\partial_{x}^{\alpha_{0}} \partial_{y}^{\beta_{0}}\left(e^{-i k h_{1}\left(x, y^{\prime}\right)} F_{k}(x, y)\right)\right|_{\left(x_{0}, x_{0}^{\prime}\right)} .
\end{aligned}
$$

Since $h_{1}\left(x_{0}, x_{0}^{\prime}\right)=-x_{0}^{2 n-1}$ and $F_{k}\left(x, y^{\prime}\right) \equiv 0 \bmod O\left(k^{-\infty}\right)$, we have

$$
\left.\lim _{k \rightarrow \infty} k^{-n} \partial_{x}^{\alpha_{0}} \partial_{y}^{\beta_{0}}\left(e^{-i k h_{1}\left(x, y^{\prime}\right)} F_{k}\left(x, y^{\prime}\right)\right)\right|_{\left(x_{0}, x_{0}\right)}=0 .
$$

On the other hand, we can check that

$$
\begin{aligned}
& \left.\lim _{k \rightarrow \infty} k^{-n} \partial_{x}^{\alpha_{0}} \partial_{y}^{\beta_{0}}\left(e^{i k h\left(x, y^{\prime}\right)-i k h_{1}\left(x, y^{\prime}\right)} g\left(x, y^{\prime}, k\right)-b\left(x, y^{\prime}, k\right)\right)\right|_{\left(x_{0}, x_{0}^{\prime}\right)} \\
& =C_{\alpha_{0}, \beta_{0}} g_{0}\left(x_{0}, x_{0}^{\prime}\right) \neq 0
\end{aligned}
$$

since $g_{0}\left(x_{0}, x_{0}^{\prime}\right) \neq 0$. From (8.7), (8.8) and (8.9), we get a contradiction. Thus, $h\left(x, y^{\prime}\right)-$ $h_{1}\left(x, y^{\prime}\right)$ vanishes to infinite order at $\left(x_{0}, x_{0}^{\prime}\right)$. Since $x_{0}$ is arbitrary, the theorem follows.

Acknowledgements. We are most grateful to Louis Boutet de Monvel and Johannes Sjöstrand for their beautiful theory [17] and many discussions about the Szegö kernel over the years. 


\section{REFERENCES}

[1] A. Andreotti and Y. T. Siu, Projective embeddings of pseudoconcave spaces, Ann. Sc. Norm. Sup. Pisa 24 (1970), 231-278.

[2] L. Baracco, The range of the tangential Cauchy-Riemann system to a CR embedded manifold, Invent. Math. 190 (2012), no. 2, 505-510.

[3] M.-S. Baouendi and L.-P. Rothschild and F. Trèves, CR structures with group action and extendability of CR functions, Invent. math. 83 (1985), 359-396.

[4] D. Barrett, A remark on the global embedding problem for three-dimensional CR manifolds, Proc. Amer. Math. Soc. 102 (1988), 888-892.

[5] R. Berman, Bergman kernels and equilibrium measures for line bundles over projective manifolds for high powers, Amer. J. Math., 131 (2009), no. 5, 1485-1524.

[6] B. Berndtsson, Subharmonicity properties of the Bergman kernel and some other functions associated to pseudoconvex domains, Ann. Inst. Fourier (Grenoble) 56 (2006), No. 6, 1633-1622.

[7] O. Biquard, Métriques autoduales sur la boule, Invent. Math. 148 (2002), no. 3, 545-607.

[8] O. Biquard, Sur des variétés de Cauchy-Riemann dont la forme de Levi a une valeur propre positive, Math. Z. 249 (2005), no. 2, 411-425.

[9] J. Bland and T. Duchamp, Moduli for pointed convex domains, Invent. Math. 104 (1991), 61-112.

[10] H. P. Boas and M. C. Shaw, Sobolev estimates for the Lewy operator on weakly pseudoconvex boundaries, Math. Ann. 274 (1986) 221-231.

[11] M. Bordemann, E. Meinrenken, and M. Schlichenmaier, Toeplitz quantization of Kähler manifolds and $g l(N), N \longrightarrow \infty$ limits, Comm. Math. Phys. 165 (1994), 281-296.

[12] D. Borthwick and A. Uribe, Nearly Kählerian embeddings of symplectic manifolds, Asian J. Math. 4 (2000), no. 3, 599-620.

[13] D. Borthwick and T. Paul and A. Uribe, Semiclassical spectral estimates for Toeplitz operators, Ann. Inst. Fourier (Grenoble) 48 (1998), no. 4, 1189-1229.

[14] L. Boutet de Monvel, Intégration des equations de Cauchy-Riemann induites formelles, Séminaire Goulaouic-Lions-Schwartz, 1974-75, Exposé No. 9.

[15] L. Boutet de Monvel, Complément sur le noyau de Bergman, Séminaire sur les Équations aux Dérivées Partielles, 1985-1986, Exp. No. XX, 13 pp., École Polytech., Palaiseau, 1986.

[16] L. Boutet de Monvel, Le noyau de Bergman en dimension 2, Séminaire sur les Équations aux Dérivées Partielles 1987-1988, Exp. No. XXII, 13 pp., École Polytech., Palaiseau, 1988.

[17] L. Boutet de Monvel and J. Sjöstrand, Sur la singularité des noyaux de Bergman et de Szegö, Astérisque, 34-35 (1976), 123-164.

[18] L. Boutet de Monvel and V. Guillemin, The spectral theory of Toeplitz operators, Ann. of Math. Stud., vol 99, Princeton Univ. Press, Princeton, NJ, 1981.

[19] D. M. Burns, Global behavior of some tangential Cauchy-Riemann equations, Partial differential equations and geometry (Proc. Conf., Park City, Utah, 1977), Lecture Notes in Pure and Appl. Math., vol. 48, Dekker, New York, 1979, pp. 51-56.

[20] D. M. Burns and C.-L. Epstein, Embeddability of Three-Dimensional CR-Manifolds, J. Amer. Math. Soc. 4 (1990), 809-840.

[21] D. Catlin, The Bergman kernel and a theorem of Tian, in Analysis and geometry in several complex variables (Katata, 1997), 1-23, Trends Math., Birkhäuser, Boston, 1999.

[22] L. Charles, Berezin-Toeplitz operators, a semi-classical approach, Comm. Math. Phys. 239 (2003), 1-28.

[23] S-C. Chen and M-C. Shaw, Partial differential equations in several complex variables, AMS/IP Studies in Advanced Mathematics. 19. Providence, RI: American Mathematical Society (AMS). Somerville, MA: International Press, xii, 380 p., (2001).

[24] M. Christ, Embedding compact three-dimensional CR manifolds of finite type in $\mathbb{C}^{n}$. Ann. of Math. 129 (1989), no. 1, 195-213.

[25] E.-B. Davies, Spectral Theory and Differential Operators, Cambridge Stud. Adv. Math., vol. 42, (1995).

[26] S. K. Donaldson, Scalar curvature and projective embeddings. I, J. Differential Geom. 59 (2001), 479-522.

[27] H. Donnelly, Spectral theory for tensor products of Hermitian holomorphic line bundles, Math. Z. 245, (2003), no. 1, 31-35. 
[28] T. C. Dinh and G. Marinescu and V. Schmidt, Asymptotic distribution of zeros of holomorphic sections in the non compact setting, J. Stat. Phys. 148 (2012), no. 1, 113-136.

[29] M. Engliš, Weighted Bergman kernels and quantization, Comm. Math. Phys. 227 (2002), no. 2, $211-241$.

[30] C. L. Epstein, CR-structures on three dimensional circle bundles, Invent. Math. 109 (1992), 351-403.

[31] C. L. Epstein, Subelliptic boundary conditions for Spin ${ }^{c}$-Dirac operators, gluing, relative indices, and tame Fredholm pairs, Proc. Natl. Acad. Sci. USA 103 (2006), no. 42, 15364-15369.

[32] C. L. Epstein, Addendum to: Subelliptic Spin $\mathbb{C}$ Dirac operators, III, Ann. of Math. 176 (2012), no. 2, 13731380.

[33] Ch. Fefferman, The Bergman kernel and biholomorphic mappings of pseudoconvex domains, Invent. Math. 26 (1974), No. 1, 1-65.

[34] G. B. Folland, J. J. Kohn, The Neumann problem for the Cauchy-Riemann complex. Annals of Mathematics Studies, No. 75. Princeton University Press, Princeton, N.J.; University of Tokyo Press, Tokyo, 1972. viii+146 pp.

[35] H. Grauert, Über Modifikationen und exzeptionelle analytische Mengen, Math. Ann. 146 (1962), 331-368.

[36] H. Grauert, Theory of q-convexity and q-concavity, Several Complex Variables VII (H. Grauert, Th. Peternell, and R. Remmert, eds.), Encyclopedia of mathematical sciences, vol. 74, Springer Verlag, 1994.

[37] A. Grigis and J. Sjöstrand, Microlocal analysis for differential operators. An introduction, London Mathematical Society Lecture Note Series, vol. 196, Cambridge University Press, Cambridge, 1994.

[38] V. Guillemin, Star products on compact pre-quantizable symplectic manifolds, Lett. Math. Phys. 35 (1995), no. $1,85-89$.

[39] F. R. Harvey and H. B. Lawson, On boundaries of complex analytic varieties. I. Ann. of Math. (2) 102 (1975), no. 2, 223-290.

[40] K. Hirachi, Logarithmic singularity of the Szegó kernel and a global invariant of strictly pseudoconvex domains, Ann. of Math. (2) 163 (2006), no. 2, 499-515.

[41] L. Hörmander, An introduction to complex analysis in several variables, North-Holland mathematical library, North-Holland Publishing Co., Amsterdam, 7, 1990.

[42] L. Hörmander, The analysis of linear partial differential operators. I, Classics in Mathematics, SpringerVerlag, Berlin, 2003.

[43] L. Hörmander, The analysis of linear partial differential operators. III, Grundlehren der Mathematischen Wissenschaften, Springer-Verlag, Berlin, 274, (1985).

[44] C.-Y. Hsiao, Projections in several complex variables, Mém. Soc. Math. France, Nouv. Sér. 123 (2010), 131 p.

[45] C-Y. Hsiao, Existence of CR sections for high power of semi-positive generalized Sasakian CR line bundles over generalized Sasakian CR manifolds, 46 pages, preprint available at arXiv:1204.4810.

[46] C.-Y. Hsiao, Szegö kernel asymptotics for high power of CR line bundles and Kodaira embedding theorems on CR manifolds, 116 pages, preprint available at arXiv:1401.6647.

[47] C-Y. Hsiao and G. Marinescu, Szegö kernel asymptotics and Morse inequalities on CR manifolds, Math. Z. 271 (2012), 509-553.

[48] C-Y. Hsiao and G. Marinescu, Asymptotics of spectral function of lower energy forms and Bergman kernel of semi-positive and big line bundles, Commun. Anal. Geom. 22 (2014), No. 1, 1-108.

[49] M. Kashiwara, Analyse micro-locale du noyau de Bergman, Séminaire Goulaouic-Schwartz (1976/1977), Équations aux dérivées partielles et analyse fonctionnelle, Exp. No. 8, 10 pp. Centre Math., École Polytech., Palaiseau, 1977

[50] J. J. Kohn, Boundaries of complex manifolds, Proc. Conf. Complex Analysis (Minneapolis, 1964), pp. 81-94, Springer, Berlin, 1965.

[51] J. J. Kohn, Estimates for $\bar{\partial}_{b}$ on pseudoconvex CR manifolds, Proc. Symp. in Pure Math. 43 (1985), $207-217$.

[52] J. J. Kohn, The range of the tangential Cauchy-Riemann operators, Duke Math. J. 53 (1986), No. 2, 307562.

[53] L. Lempert, On three dimensional Cauchy-Riemann manifolds, J. Amer. Math. Soc. 5 (1992), 1-50.

[54] Z. Lu and G. Tian, The log term of the Szegö kernel, Duke Math. J. 125 (2004), no. 2, 351-387.

[55] X. Ma, Geometric quantization on Kähler and symplectic manifolds, Proceedings of the international congress of mathematicians (ICM 2010), Hyderabad, India, August 19-27, 2010. Vol II, 785-810. 
[56] X. Ma and G. Marinescu, The first coefficients of the asymptotic expansion of the Bergman kernel of the spin ${ }^{c}$ Dirac operator, Internat. J. Math. 17 (2006), no. 6, 737-759.

[57] X. Ma and G. Marinescu, Holomorphic Morse inequalities and Bergman kernels, Progress in Math., vol. 254, Birkhäuser, Basel, 2007, 422 pp.

[58] X. Ma and G. Marinescu, Generalized Bergman kernels on symplectic manifolds, Adv. Math. 217 (2008), no. 4, 1756-1815.

[59] G. Marinescu and N. Yeganefar, Embeddability of some strictly pseudoconvex manifolds, Trans. Amer. Math. Soc. 359 (2007), 4757-4771.

[60] A. Melin and J. Sjöstrand, Fourier integral operators with complex-valued phase functions, Springer Lecture Notes in Math., 459 (1975), 120-223.

[61] A. Nicoară, Global regularity for $\bar{\partial}_{b}$ on weakly pseudoconvex CR manifolds, Adv. Math. 199 (2006), no. 2, 356-447.

[62] H. Rossi, Attaching analytic spaces to an analytic space along a pseudoconcave boundary, Proc. Conf. Complex. Manifolds (Minneapolis), Springer-Verlag, New York, 1965, pp. 242-256.

[63] M-C. Shaw, $L^{2}$ estimates and existence theorems for the tangential Cauchy-Riemann complex, Invent. math. 82 (1985), 133-150.

[64] B. Shiffman and S. Zelditch, Distribution of zeros of random and quantum chaotic sections of positive line bundles, Comm. Math. Phys. 200 (1999), 661-683.

[65] M. E. Taylor, Partial differential equations. 1: Basic theory, Applied Mathematical Sciences, vol. 115, Springer-Verlag, Berlin, 1996.

[66] S. Zelditch, Szegó kernels and a theorem of Tian, Int. Math. Res. Not. 6 (1998), 317-331.

Institute of Mathematics, Academia Sinica, 6F, Astronomy-Mathematics Building, No.1, Sec.4, ROOSEVELT ROAD, TAIPEI 10617, TAIWAN

E-mail address: chsiao@math.sinica.edu.tw or chinyu.hsiao@gmail.com

Universität Zu KÖLn, Mathematisches Institut, Weyertal 86-90, 50931 KÖLn, Germany, \& InstiTUte of Mathematics 'Simion STOILOW', Romanian ACADEMY, BuCHAREST, Romania

E-mail address: gmarines@math. uni-koeln.de 